\title{
Resultados de Ramsey e de densidade para grafos pseudo-aleatórios esparsos
}

JAIR DONADELLI JÚNIOR

\author{
TESE APRESENTADA \\ $\mathrm{AO}$ \\ INSTITUTO DE MATEMÁTICA E ESTATÍSTICA \\ DA \\ UNIVERSIDADE DE SÃO PAULO \\ PARA \\ OBTENÇÃO DO GRAU DE DOUTOR \\ EM \\ MATEMÁTICA APLICADA
}

\begin{abstract}
Área de Concentração: Ciência da Computação
Orientador: Prof. Dr. Yoshiharu Kohayakawa
\end{abstract}

Durante a elaboração deste trabalho o autor recebeu apoio financeiro do CNPq e da CAPES

-São Paulo, dezembro de 2001- 


\section{Resultados de Ramsey e de densidade para grafos pseudo-aleatórios esparsos}

Este exemplar corresponde à redação final da tese devidamente corrigida e defendida por Jair Donadelli Júnior e aprovada pela comissão julgadora.

São Paulo, 4 de abril de 2002

Banca examinadora:

Prof. Dr. Yoshiharu Kohayakawa (orientador) IME-USP

Profa. Dra. Cristina Gomes Fernandes IME-USP

Prof. Dr. Carlos Gustavo Tamm de A. Moreira IMPA

Prof. Dr. Vojtech Rödl

EMORY UNIVERSITY

Prof. Dr. Hanno Lefmann

TECHNISCHE UNIVERSITÄT CHEMNITZ 

À minha família. 


$$
\begin{aligned}
& \because \ldots+\therefore, \quad \therefore \therefore \therefore \ldots
\end{aligned}
$$

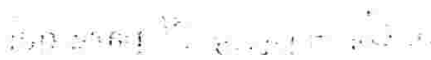

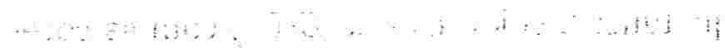

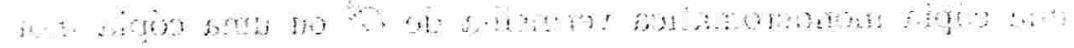




\section{Resumo}

Neste trabalho estudamos problemas do tipo ramsey e do tipo densidade para grafos e grafos orientados esparsos.

Começamos dedicando o primeiro capítulo a uma das principais ferramentas no ataque aos problemas: o Lema de Regularidade de Szemerédi.

Na seqüência, provamos uma generalização de um lema de contagem devido a Kohayakawa e Kreuter (1997). Com esse resultado, aplicamos um método inventado por Füredi para provar que quase certamente $\vec{G}_{p} \rightarrow_{1 / 2+\beta} \vec{C}^{\ell}$ para qualquer $\beta>0$, onde $\vec{G}_{p}$ é o grafo orientado aleatório binomial de ordem $n$ e densidade $p=e\left(\vec{G}_{p}\right) / n^{2}=A n^{-1+1 /(\ell-1)}$, e $A=A(\beta)>0$ é uma constante suficientemente grande e $\vec{G} \rightarrow_{1 / 2+\beta} \vec{C}^{\ell}$ significa que todo subgrafo $\vec{J} \subseteq \vec{G}$ com $e(\vec{J}) \geqslant(1 / 2+\beta) e(\vec{G})$ contém um circuito $\vec{C}^{\ell}$. Como conseqüência disso obtemos uma família infinita de contra-exemplos para uma generalização de uma conjectura de Woodall.

Depois, usamos o lema de contagem de circuitos para mostrar que para todo grafo $H$ de uma família apropriada de grafos 2-conexos e para todo $n$ suficientemente grande, existem grafos $\Gamma^{n}$ tais que $\Gamma^{n} \rightarrow\left(C^{\ell}, H\right)$ e $\Gamma^{n}$ é minimal com respeito a essa propriedade. O símbolo $\Gamma^{n} \rightarrow\left(C^{\ell}, H\right)$ significa que para qualquer 2-coloração de $E\left(\Gamma^{n}\right)$ com as cores vermelha e azul, ou teremos uma cópia monocromática vermelha de $C^{\ell}$ ou uma cópia monocromática azul de $H$ contida em $\Gamma^{n}$.

Finalmente, demonstramos que para qualquer grafo $H$, se subdividimos suas arestas $s$ vezes, onde $C_{1} \log n \leq s \leq C_{2} n$, então o número de ramsey para aresta para o grafo resultante é $O(n)$. Esse resultado está relacionado com uma conjectura recente de Igor Pak. 


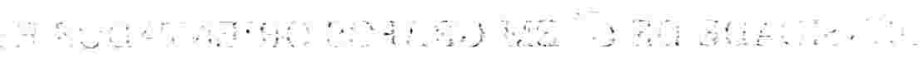

a d

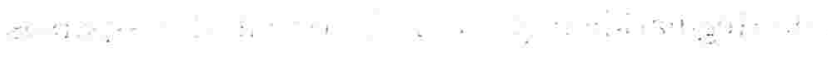

$\therefore \sin 2 \cos$

$\therefore$. A 


\section{Sumário}

INTRODUÇÃO

Capítulo 0. O LEMA DE REGULARIDADE DE SZEMERÉDI 35

1. Introdução 35

2. Uma versão para grafos esparsos do Lema de Szemerédi 42

3. Uma demonstração do Lema de Szemerédi 44

4. Considerações finais 50

Capítulo 1. CONTAGEM DE $C^{\ell}$ 's EM GRAFOS ESPARSOS 51

1. Introdução 51

2. Lema de Contagem 54

3. Considerações finais 56

Capítulo 2. A DENSIDADE DE $\vec{C}^{\ell}$ EM GRAFOS ORIENTADOS ESPARSOS E UMA $\begin{array}{ll}\text { CONJECTURA DE WOODALL } & 59\end{array}$

1. Introdução $\quad 59$

2. Um Lema de Regularidade para grafos orientados esparsos 62

3. A versão do Lema de Contagem para grafos orientados 63

4. A demonstração do teorema principal 64

5. Considerações finais $\quad 70$

Capítulo 3. GRAFOS $\left(C^{\ell}, H\right)$-RAMSEY MINIMAIS 73

1. Introdução 73

2. A construção do grafo $\Gamma=\Gamma^{n}(\ell, t, H) \quad 77$

3. A demonstração do Teorema 33

4. Sobre a Propriedade (P6) 87 
5. Considerações finais

Capítulo 4. O NÚMERO DE RAMSEY-ARESTA DE SUBDIVISÕES DE GRAFOS 91

1. Introdução 91

2. Resultados auxiliares 94

3. Demonstração do teorema principal 100

4. Grafos $(p, A)$-uniformes 105

5. Considerações finais 107

Referências Bibliográficas 109

Índice Remissivo 


\section{INTRODUÇÃO}

O Teorema de Ramsey (Ramsey, 1930) diz que, para todos $m$ e $r$ inteiros e positivos, existe um inteiro positivo $n$ tal que não importa como $r$-colorimos as arestas do grafo completo com $n$ vértices, denotado por $K^{n}$, sempre temos um $K^{m}$ monocromático, isto é, um grafo completo sobre um subconjunto com $m$ vértices do $K^{n}$ e com todas as arestas da mesma cor. Em notação atual

$$
K^{n} \rightarrow\left(K^{m}, \ldots, K^{m}\right),
$$

onde $\left(K^{m}, \ldots, K^{m}\right)$ é uma seqüência com $r$ ocorrências do $K^{m}$. Mais geralmente, sabemos que dados os grafos $H_{1}, \ldots, H_{r}(r \geqslant 1)$, existe um grafo $\Gamma$ tal que $\Gamma \rightarrow\left(H_{1}, \ldots, H_{r}\right)$, ou seja, para qualquer $r$-coloração das arestas de $\Gamma$, existe um subgrafo $\tilde{H} \subseteq \Gamma$ isomorfo à $H_{i}$ e com todas as suas arestas da cor $i$, para algum $1 \leqslant i \leqslant r$.

Por exemplo $K^{3,7} \rightarrow\left(C^{4}, C^{4}\right)$, onde $K^{3,7}$ é o grafo bipartido com $3+7$ vértices e todas as $3 \times 7$ arestas, e $C^{4}$ é o circuito com quatro vértices. De fato, considere uma coloração qualquer das arestas do $K^{3,7}$ com duas cores, digamos branca e preta. Seja $T=\{x, y, z\}$ a parte do conjunto de vértices do $K^{3,7}$ com três vértices. Sem perda de generalidade, podemos supor que nos vértices $x, y \in T$ incidem quatro arestas da mesma cor, digamos preta. Se incidem mais que quatro arestas, os vértices $x$ e $y$ teriam pelo menos dois vizinhos em comum e teríamos um $C^{4}$ com todas as arestas da cor preta. Assim, podemos supor também que eles têm um único vizinho comum (veja Figura 1). Se em $z$ incidem três ou mais arestas pretas, temos um $C^{4}$ monocromático da cor preta, portanto, podemos supor que em $z$ incidem pelo menos cinco arestas brancas. Nesse caso, teremos um $C^{4}$ monocromático da cor branca pois em $x$ e em $y$ incidem três arestas brancas cada. 


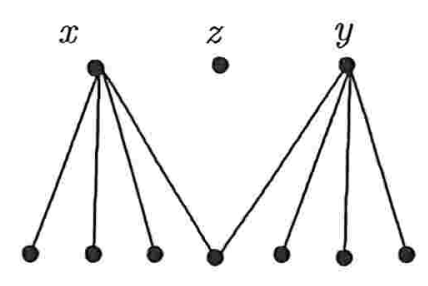

Figura $1 . K^{3,7} \rightarrow\left(C^{4}, C^{4}\right)$

Lembramos que o número cromático de um hipergrafo $\mathcal{G}$, denotado por $\chi(\mathcal{G})$, é o menor número de partes em que podemos dividir o conjunto de vértices de $\mathcal{G}$ de modo que nenhuma das partes induza uma hiperaresta.

O resultado de Ramsey acima pode ser escrito na linguagem de hipergrafos da seguinte forma: considere o hipergrafo $\mathcal{G}=\mathcal{G}\left(K^{n} ; K^{m}\right)$, cujo conjunto de vértices é o conjunto de arestas do $K^{n}$, ou seja $V(\mathcal{G})=E\left(K^{n}\right)$, e as hiperarestas são dadas pelos subconjuntos de $E\left(K^{n}\right)$ que formam cópia de um $K^{m}$, ou seja, $E(\mathcal{G})$ é dado pelos subconjuntos $\left\{e_{1}, \ldots, e_{\left(\begin{array}{c}m \\ 2\end{array}\right)}\right\} \subseteq$ $E\left(K^{n}\right)$ que definem um grafo completo sobre $m$ vértices no $K^{n}$.

Agora, a afirmação para todos $m, r \in \mathbb{N}$ existe $n_{0} \in \mathbb{N}$ tal que $K^{n} \rightarrow\left(K^{m}, \ldots, K^{m}\right)$, para todo $n \geqslant n_{0}$, pode ser dita em linguagem de hipergrafos como: para todos $m, r \in \mathbb{N}$ existe $n_{0} \in \mathbb{N}$ tal que $\chi(\mathcal{G})>r$, para todo $n \geqslant n_{0}$. Dado um hipergrafo $\mathcal{G}$, a afirmação $\chi(\mathcal{G})>r$ é o que chamamos de um resultado do tipo ramsey.

Generalizando esses conceitos, dados os grafos $\Gamma$ e $H$, definimos o hipergrafo $\mathcal{G}=\mathcal{G}(\Gamma ; H)$ cujos vértices são as arestas de $\Gamma$ e as hiperarestas são definidas pelas arestas das cópias de $H$ em $\Gamma$. Então $\Gamma \rightarrow(H, \ldots, H)$ se, e somente se, $\chi(\mathcal{G})>r$.

Dado um hipergrafo $\mathcal{G}=(V, E)$, seja $T(\mathcal{G})$ o menor $t \in \mathbb{N}$ tal que qualquer subconjunto de vértices com pelo menos $t$ elementos contém uma aresta de $\mathcal{G}$. Definimos $\tau(\mathcal{G})$ pondo $\tau(\mathcal{G})=T(\mathcal{G}) /|V(\mathcal{G})|$. Observamos que se $\tau(\mathcal{G}) \leqslant r^{-1}$, então $\chi(\mathcal{G})>r$, mas a recíproca não vale (veja Graham et al., 1980, página 13). Dado um hipergrafo $\mathcal{G}$, a afirmação $\tau(G) \leqslant r^{-1}$ é o que chamamos de um resultado do tipo densidade.

O menor $t \in \mathbb{N}$ tal que qualquer conjunto de vértices de $\mathcal{G}\left(K^{n} ; K^{m}\right)$ com pelo menos $t$ elementos contém uma cópia de $K^{m}$ é, em linguagem de Teoria dos Grafos, o número de Turán ex $\left(n, K^{m}\right)$ mais um (veja Teorema 6, página 29). De modo análogo, para os grafos $\Gamma$ 
e $H$ nós definimos $\tau(\mathcal{G})$, para $\mathcal{G}=\mathcal{G}(\Gamma ; H)$. E, em notação atual, $\tau(\mathcal{G})=\gamma$ é escrito como $\Gamma \rightarrow_{\gamma} H$.

Por exemplo, um dos primeiros resultados do tipo ramsey em combinatória foi demonstrado por van der Waerden em 1927.

Teorema 1 (van der Waerden, 1927). Para todos $\ell, r \in \mathbb{N}$ existe $n_{0}(\ell, r) \in \mathbb{N}$ tal que para todo inteiro $n \geqslant n_{0}(\ell, r)$ vale o seguinte: em qualquer $r$-coloração de $[n]$ ocorre uma progressão aritmética monocromática composta por $\ell$ termos.

E o resultado análogo do tipo densidade, que enunciamos a seguir, é o famoso Teorema de Szemerédi sobre progressões aritméticas.

TeOrema 2 (Szemerédi, 1975). Para todo $\ell \in \mathbb{N}$ e todo real $\varepsilon>0$ existe $n_{0}(\ell, \varepsilon) \in \mathbb{N}$ tal que para todo inteiro $n \geqslant n_{0}(\ell, \varepsilon)$ vale o seguinte: todo subconjunto $A \subseteq[n] \operatorname{com}|A| \geqslant \varepsilon n$ contém uma progressão aritmética de $\ell$ termos.

Para demonstrar esse resultado, Szemerédi provou o que hoje é conhecido como Lema de Regularidade de Szemerédi, ao qual é dedicado o Capítulo 0. De uma maneira superficial, o lema diz que o conjunto de vértices de todo grafo pode ser particionado em $k$ partes com cardinalidades que diferem de no máximo um, de modo que uma fração próxima de um dos $\left(\begin{array}{l}k \\ 2\end{array}\right)$ subgrafos bipartidos induzidos pela partição são pseudo-aleatórios, um conceito que não definiremos agora, mas que, entre outras coisas, diz que esses subgrafos se comportam localmente como se fossem grafos bipartidos aleatórios. O Lema de Regularidade trouxe muitas conseqüências expressivas no desenvolvimento da Teoria Extremal de Grafos e é atualmente uma ferramenta muito importante, como pode ser visto na resenha éscrita por Komlós e Simonovits (1996) e em recentes livros-texto sobre Teoria dos Grafos (Diestel, 1997; Bollobás, 1998).

Em todos os principais resultados deste trabalho usamos o Lema de Regularidade. Porém, o lema original de Szemerédi não nos fornece informações úteis quando o grafo alvo da aplicação do lema é esparso, isto é, quando o número de arestas é da ordem de $n^{2-\alpha}$, onde $\alpha$ é uma constante positiva e $n$ o número de vértices do grafo. De fato, a pseudo-aleatoriedade 
envolvida nos pares regulares tem relação com a distribuição das arestas, essas devem estar distribuídas uniformemente com um termo de erro que é quadrático no número de vértices, mas com uma constante multiplicativa arbitrariamente pequena.

Como alternativa para o caso esparso, Kohayakawa (1993) e Rödl provaram, independentemente, uma versão para grafos pseudo-aleatórios esparsos que tem tido bastante sucesso, inclusive em resultados do tipo ramsey e do tipo densidade (veja, por exemplo Haxell et al., 1995a,b; Kohayakawa et al., 1996; Haxell et al., 1996; Kohayakawa et al., 1997; Kohayakawa e Kreuter, 1997; Kohayakawa e Rödl, 2001).

Voltando aos resultados do tipo ramsey e do tipo densidade, uma questão que surge naturalmente é investigar qual o comportamento típico de grafos quanto aos resultados do tipo densidade e do tipo ramsey, ou seja, qual o comportamento de $\mathbb{G} \rightarrow_{\gamma} H$ e $\mathbb{G} \rightarrow(G, H)$, onde $G$ e $H$ são grafos quaisquer e $\mathbb{G}$ é um modelo de grafo aleatório.

Se denotamos por $\mathcal{G}(n, M)$ o conjunto de todos os grafos com $n$ vértices e $M=M(n)$ arestas e por $\mathcal{G}\left(n, M ; C^{4}\right)$ o subconjunto desses que não contêm circuito $C^{4}$, então sabemos que (veja Janson et al., 2000, Seção 3.1)

$$
\lim _{n \rightarrow \infty} \frac{\left|\mathcal{G}\left(n, M ; C^{4}\right)\right|}{|\mathcal{G}(n, M)|}= \begin{cases}0 & \text { se } M \gg n \\ 1 & \text { se } M \ll n\end{cases}
$$

Portanto, se escolhemos $G_{n, M} \in \mathcal{G}(n, M)$ aleatoriamente com distribuição uniforme, então $\mathbb{P}\left\{G_{n, M} \nsupseteq C^{4}\right\}<\varepsilon$, para todo $\varepsilon>0$, todo $M \geqslant n^{4 / 3}$ e todo $n$ suficientemente grande. Além disso, se $M \leqslant c n^{4 / 3}$, onde $c$ é constante positiva, então

$$
\mathbb{P}\left\{G_{n, M} \nsupseteq C^{4}\right\} \leqslant \mathrm{e}^{-a M},
$$

onde $a>0$ é constante. Mas, esse fato não garante que todo subgrafo de $G_{n, M} \operatorname{com} M^{\prime}=\beta M$ arestas contenha um $C^{4}$, pois $\mathbb{P}\left\{G_{n, M^{\prime}} \nsupseteq C^{4}\right\} \leqslant \mathrm{e}^{-a^{\prime} M}$, onde $a^{\prime}=a^{\prime}(a, \beta)>0$ é uma constante, e existem por volta de $2^{M}$ desses subgrafos. 
Nesse caso particular, o de circuito com quatro vértices, Füredi (1994) mostrou que $\mathbb{P}\left\{G_{n, M} \nsupseteq C^{4}\right\}=o(1)^{M}$, para todo $M \gg n^{4 / 3}$. A técnica inventada por Füredi, de mostrar que o número de contra-exemplos para uma determinada propriedade é superexponencialmente pequeno - logo a propriedade quase-certamente não ocorre em nenhum subgrafo de $\mathbb{G}$ - tem sido útil em vários casos (Haxell et al., 1995b; Kohayakawa et al., 1996, 1997, 1998; Kohayakawa e Kreuter, 1997; Kohayakawa e Rödl, 2001) e é brevemente discutida no Capítulo 1, onde provamos uma generalização de um resultado demonstrado por Kohayakawa e Kreuter (1997) sobre contagem de circuitos $C^{\ell}$ em grafos $\ell$-partidos esparsos.

O resultado de Kohayakawa e Kreuter (1997) para $C^{4}$ pode ser enunciado como segue. Seja $\mathcal{B}(\varepsilon, m, M)$ a família dos grafos bipartidos $B(U, W ; E)$, onde $|U|=|W|=m$, com $|E|=T$ arestas e $(\varepsilon, T)$-regulares, isto é, tais que

$$
|| E(A, B)|-| A|| B\left|\frac{T}{m^{2}}\right| \leqslant \varepsilon|A||B| \frac{T}{m^{2}}
$$

para todos $A \subseteq U$ e $B \subseteq W$ com $|A|,|B| \geqslant \varepsilon m$. Denotando por $\mathcal{B}\left(\varepsilon, m, T ; C^{4}\right)$ a família dos grafos bipartidos $(\varepsilon, T)$-regulares que não contêm $C^{4}$, temos o seguinte resultado de contagem.

Dado $\alpha>0$ existem $c=c(\alpha), m_{0}=m_{0}(\alpha)$ e $\varepsilon=\varepsilon(\alpha)>0$ tais que para todo $m \geqslant m_{0}$ se $T \geqslant \mathrm{~cm}^{4 / 3}$, então

$$
\frac{\left|\mathcal{B}\left(\varepsilon, m, T ; C^{4}\right)\right|}{|\mathcal{B}(\varepsilon, m, T)|} \leqslant \alpha^{T}
$$

Esse resultado juntamente com o Lema de Szemerédi garante que a probabilidade de subgrafos bipartidos grandes do $G_{n, M}$ não conterem $C^{4}$ é superexponencialmente pequena, para $M$ "não muito grande" e com as arestas do $G_{n, M}$ regularmente distribuídas. Logo, $G_{n, M}$ deve conter $C^{4}$ 's de uma maneira robusta, ou seja, todo subgrafo com uma proporção grande das arestas deve conter um circuito.

No Capítulo 2, usamos o Lema de Regularidade e a técnica de Füredi para demonstrar um resultado de densidade com respeito a circuitos em grafos orientados esparsos. Esse resultado para $\vec{C}^{4}$ diz que existe $\vec{G}$ com $n$ vértices e $O\left(n^{4 / 3}\right)$ arcos e que contém $\vec{C}^{4}$ de 
maneira robusta, para todo $n$ suficientemente grande. A prova segue as observações feitas anteriormente, especializadas para tratar de grafos orientados.

Escrevemos $\vec{G}^{n}$ para um grafo orientado com $n$ vértices. O nosso resultado (Donadelli e Kohayakawa, 2000) do Capítulo 2 é o seguinte:

Dados um inteiro $\ell \geqslant 3$ e um real $\beta>0$ existe um grafo orientado $\vec{G}^{n}$, para todo $n$ suficientemente grande, com $O\left(n^{1+1 /(\ell-1)}\right)$ arcos e cintura orientada $\ell$ tal que qualquer subgrafo de $\vec{G}^{n}$ com mais que $(1 / 2+\beta)\left|E\left(\vec{G}^{n}\right)\right|$ arcos contém um circuito orientado de comprimento $\ell$.

Esse resultado é o melhor possível no seguinte sentido: qualquer grafo orientado $\vec{G}$ contém um subgrafo sem circuitos orientados e com pelo menos metade de seus arcos, como pode ser visto tomando-se uma ordem total qualquer no conjunto de vértices $V(\vec{G})$. Além disso, deduziremos desse resultado que existem infinitos contra-exemplos para uma generalização de uma conjectura de Woodall sobre transversais de circuitos em grafos orientados planares. Queremos deixar claro que já era conhecido um contra-exemplo devido a Thomassen para tal conjectura. A principal diferença é que o contra-exemplo de Thomassen é um torneio construído sobre 15 vértices, ou seja é um grafo orientado com todas as arestas possíveis, enquanto que nossos contra-exemplos são muito esparsos e mostram que tais contra-exemplos são abundantes.

No começo desta introdução vimos que $K^{3,7} \rightarrow\left(C^{4}, C^{4}\right)$. Na verdade, quase todo grafo com $n$ vértices e $\Omega\left(n^{4 / 3}\right)$ arestas satisfaz a propriedade " $\rightarrow\left(C^{4}, C^{4}\right)$ ". Esse é um caso particular de um resultado de Rödl e Ruciński (1995), o Teorema 3 na página 22, que pode ser ingenuamente justificado da seguinte maneira.

Dado $M \geqslant A n^{4 / 3}$, para alguma constante $A>0$, seja $\mathcal{H}=\mathcal{H}_{n, M}$ um hipergrafo 4-uniforme sobre o conjunto de vértices $[n]$ escolhido aleatoriamente com distribuição uniforme no conjunto de todos hipergrafos 4-uniformes com $M$ arestas. Mergulhamos em cada hiperaresta de $\mathcal{H}$ uma cópia de $C^{4}$ arbitrariamente, e denotamos por $\Gamma$ o grafo resultante.

Agora, escolhemos uma aresta em cada hiperaresta de $\mathcal{H}$ para pintar de azul; assim esperamos destruir a possibilidade de existirem cópias azuis de $C^{4}$ nesse grafo $\Gamma$. Ainda, podemos esperar que tal subgrafo azul de $\Gamma$ se "comporte como" $G_{n, \bar{M}}$, onde $\bar{M} \geqslant \bar{A} n^{4 / 3}$ e, portanto, 
a probabilidade desse subgrafo azul conter um circuito $C^{4}$ é maior que $1 / 2$, se a constante $\bar{A}=\bar{A}(A)$ for suficientemente grande. Ou seja, temos a propriedade " $\Gamma \rightarrow\left(C^{4}, C^{4}\right)$ ". Rödl e Ruciński (1995) ainda deduzem, como um corolário, a existência de grafos localmente esparsos, num sentido que fazemos preciso abaixo, e satisfazendo a propriedade " $\rightarrow\left(C^{4}, C^{4}\right)$ ".

Dado um grafo $H$, definimos

$$
m(H)=\max \left\{\frac{e(J)}{v(J)}: J \subseteq H, v(J)>0\right\}
$$

Então, o resultado a que nos referimos no final do parágrafo anterior é: seja $M=C n^{4 / 3}$, onde $C$ é a constante do Teorema 3, página 22. Para todo $t \in \mathbb{N}$, quase todo $G \in \mathcal{G}(n, M)$ é tal que nenhum subgrafo $H \subset G \operatorname{com} 2<v(H) \leqslant t$ satisfaz $m(H) \geqslant 3 / 2$. Agora, seja $G$ tal como na afirmação anterior e vejamos que, se $H$ é um subgrafo de $G$ minimal com respeito a propriedade " $\rightarrow\left(C^{4}, C^{4}\right)$ ", então $H$ deve ter mais que $t$ vértices.

Para tal, basta verificarmos que, se $H \rightarrow\left(C^{4}, C^{4}\right)$, então $m(H)>3 / 2$. Conseqüentemente, $v(H)>t$. Definimos

$$
m_{1}(H)=\max \left\{\frac{e(J)}{v(J)-1}: J \subseteq H, v(J)>1\right\}
$$

Suponha que $m(H) \leqslant 3 / 2$. Um resultado de Nash-Williams (veja Diestel, 1997, Teorema 3.5.4) diz que $\left\lceil m_{1}(H)\right\rceil$ é o menor número de florestas em que podemos particionar $E(H)$ : Mas, $m_{1}(H) \leqslant m(H)+1 / 2 \leqslant 2$, ou seja, podemos 2-colorir as arestas de $H$ de modo que as cores induzem florestas em $H$, isto é, $H \nrightarrow \nrightarrow\left(C^{4}, C^{4}\right)$.

A moral da história é que para todo $t \in \mathbb{N}$ existem grafos com mais que $t$ vértices e que são minimais com respeito à propriedade " $\rightarrow\left(C^{4}, C^{4}\right)$ ". Um par de grafos $(G, H)$ que satisfaz essa propriedade é chamado de ramsey-infinito. Por exemplo, considerando agora o caminho com três vértices, denotado por $P^{3}$, então $C^{2 \ell+1} \rightarrow\left(P^{3}, P^{3}\right)$, logo a família dos circuitos ímpares mostra que existem infinitos grafos que são ramsey e minimais com relação ao par $\left(P^{3}, P^{3}\right)$.

Por outro lado, se $m$ e $n$ são números ímpares, então o par de estrelas $\left(K^{1, m}, K^{1, n}\right)$ com $m$ e $n$ arestas, respectivamente, é ramsey-finito (Burr et al., 1981a). 
De fato, todo grafo $k$-regular (ou seja, todos os vértices têm grau $k$ ) de grau par contém um subgrafo gerador 2-regular (Diestel, 1997, Corolário 2.1.5), mais do que isso, o grafo pode ser decomposto em subgrafos geradores, disjuntos nas arestas e 2-regulares, chamados de 2-fatores. Assim, qualquer grafo $J$ que é $(m+n-2)$-regular admite a seguinte (vermelho,azul)-coloração das arestas sem cópia vermelha do $K^{1, m}$, nem cópia azul do $K^{1, n}$ : colorimos $(m-1) / 2$ dos 2 -fatores de vermelho e $(n-1) / 2$ deles de azul. Portanto, um grafo $J$ que é ramsey com relação ao par $\left(K^{1, m}, K^{1, n}\right)$ deve ter grau máximo pelo menos $m+n-1$, pois, se tiver grau máximo menor que isso, mergulhamos $J$ num grafo $(m+n-2)$-regular e usamos a 2-coloração dada acima.

Claramente, o grafo $K^{1, m+n-1}$ é $\left(K^{1, m}, K^{1, n}\right)$-ramsey-minimal. Agora, se $J$ é $\left(K^{1, m}, K^{1, n}\right)$ ramsey-minimal, então $J$ tem grau máximo pelo menos $m+n-1$, portanto $J \supseteq K^{1, m+n-1}$.

O resultado geral de Rödl e Ruciński é que o par $(H, H)$ é ramsey-infinito para todo $H$ que contém circuito. Esse resultado, embora não tenha aparecido explicitamente, pode ser facilmente deduzido dos teoremas que seguem. Antes, definimos

$$
m_{2}(H)=\max \left\{\frac{e(J)-1}{v(J)-2}: J \subseteq H, v(J)>2\right\} .
$$

TeOrema 3 (Rödl e Ruciński, 1995). Para todo grafo H cujas componentes conexas não são estrelas nem caminhos com três arestas existem constantes positivas $C$ e $c$ tais que

$$
\lim _{n \rightarrow \infty} \frac{|\mathcal{R}(n, M ; H)|}{|\mathcal{G}(n, M)|}=\left\{\begin{array}{lc}
1 & \text { se } M>C n^{2-1 / m_{2}(H)} \\
0 & \text { se } M<c n^{2-1 / m_{2}(H)}
\end{array}\right.
$$

onde $\mathcal{R}(n, M ; H)$ é a família de todos os grafos com $n$ vértices e $M$ arestas que são ramsey com respeito ao $\operatorname{par}(H, H)$.

Teorema 4 (Rödl e Ruciński, 1993). Para todo grafo $F$, se $m(F) \leqslant m_{2}(H)$ então $F \nrightarrow$ $(H, H)$.

O Teorema 3 diz que $n^{2-1 / m_{2}(H)}$ é o limiar para o surgimento da propriedade " $\rightarrow(H, H)$ ". Quando as componentes conexas de $H$ são estrelas, o número relevante para o limiar é o grau máximo de $H$. Quando pelo menos uma componente de $H$ é um caminho com três arestas, a situação é um pouco diferente: o limite acima é um, quando $M \geqslant C n$ e zero, quando $M \ll n$. 
Vamos deduzir desses dois teoremas acima que para todo $t \in \mathbb{N}$ existe um grafo $(H, H)$ ramsey-minimal com mais que $t$ vértices. Denotamos por $\mathcal{H}(t, H)$ a família dos grafos $J$ tais que $v(J) \leqslant t$ e $m(J)>m_{2}(H)$. Calculando o valor esperado do número de elementos de $\mathcal{H}(t, H)$ contidos em $G_{n, M}$, onde $M=C n^{2-1 / m_{2}(H)}$ e $C$ é a constante do Teorema 3 , temos pela desigualdade de Markov que, com probabilidade tendendo a um, vale o seguinte: para todo subgrafo $J$ de $G_{n, M}$ com $\leqslant t$ vértices vale que $m(J) \leqslant m_{2}(H)$, ou seja, $J \notin \mathcal{H}(t, H)$. Pelo Teorema 4 esses subgrafos não são ramsey com relação ao par $(H, H)$.

No Capítulo 3 estudamos famílias de grafos ramsey-minimais com relação ao par $\left(C^{\ell}, H\right)$, onde $H$ é um grafo 2-conexo tal que todo circuito de comprimento pelo menos $\ell$ contém uma corda. O resultado que demonstramos (Bollobás et al., 2002) no Capítulo 3, com o auxílio do Lema de Regularidade e usando a técnica de Füredi, é o seguinte:

Dados inteiros $\ell \geqslant 4$ e $t \geqslant 1$, e dado um grafo $H \in \mathcal{C}(\ell)$, para todo $n$ suficientemente grande, existe um grafo $\Gamma^{n}$ tal que $\Gamma^{n} \rightarrow\left(C^{\ell}, H\right)$ e $\Gamma^{n}[U] \nrightarrow \rightarrow\left(C^{\ell}, H\right)$, para todo $U \subseteq V\left(\Gamma^{n}\right)$ com no máximo $t$ vértices.

Acima, $\mathcal{C}(\ell)$ é a classe dos grafos 2-conexos tais que todo circuito de comprimento pelo menos $\ell$ contém uma corda. Em outras palavras, temos que para todo $t \in \mathbb{N}$, existe um grafo $\Gamma$ tal que $\Gamma \rightarrow\left(C^{\ell}, H\right)$ e todo subgrafo de $\Gamma$ que é minimal com respeito à propriedade " $\rightarrow\left(C^{\ell}, H\right)$ " deve ter mais que $t$ vértices.

No Capítulo 4, o último deste trabalho, estamos interessados em estimar o número de ramsey para arestas: dados os grafos $H_{1}$ e $H_{2}$, definimos $\hat{r}\left(H_{1}, H_{2}\right)$ como o menor número de $\operatorname{arestas} M$ tal que existe um grafo $\Gamma$ com $M$ arestas e ramsey para o par $\left(H_{1}, H_{2}\right)$, isto é, em símbolos,

$$
\hat{r}\left(H_{1}, H_{2}\right)=\min \left\{e(\Gamma): \Gamma \rightarrow\left(H_{1}, H_{2}\right)\right\} .
$$

Vamos denotar por $\Delta(H)$ o grau máximo de um vértice do grafo $H$ e denotar por $\lambda(H)$ o menor número de vértices cobrindo todas as arestas do grafo $H$. O número de ramsey de $H$ na versão para arestas, $\hat{r}(H, H)$, satisfaz

$$
\Delta(H) \frac{\lambda(H)}{2} \leqslant \hat{r}(H, H) \leqslant\left(\begin{array}{c}
r(H, H) \\
2
\end{array}\right) .
$$


O limitante superior é óbvio e a igualdade vale para $H=K^{n}$ (segundo Erdős et al., 1978, foi mostrado por Chvátal). Como $2^{n / 2}<r\left(K^{n}, K^{n}\right)<4^{n}$ temos que $\hat{r}\left(K^{n}, K^{n}\right)$ é exponencial em $n$.

Para estrelas, esse número de ramsey é linear; de fato $\hat{r}\left(K^{1, n}, K^{1, n}\right)=2 n-1$, enquanto que $r\left(K^{1, n}, K^{1, n}\right) \in\{2 n-1,2 n\}$, dependendo da paridade de $n$.

O limitante inferior para $\hat{r}(H, H)$ dado acima tem o seguinte argumento (Beck, 1983): seja $\Gamma$ tal que $\Gamma \rightarrow(H, H)$. Definimos a seguinte 2-coloração das arestas de $\Gamma$ : as arestas que têm algum extremo de grau $\Delta(H)$ recebem a cor azul, caso contrário, vermelha. Logo, pela escolha de $\Gamma$, devemos ter uma cópia de $H \subseteq \Gamma$ com todas as arestas azuis. Como toda aresta dessa cópia de $H$ tem pelo menos um extremo com grau $\Delta(H)$ em $\Gamma$, temos que $e(\Gamma) \geqslant \Delta(H) \lambda(H) / 2$.

Erdős et al. (1978) introduziram a versão para arestas do número de ramsey e provaram vários resultados nessa direção, por exemplo,

$$
\hat{r}\left(K^{1, n}, K^{1, m}\right)=m+n-1 .
$$

Para mostrar que $\hat{r}\left(K^{1, n}, K^{1, m}\right) \leqslant m+n-1$ basta tomar uma 2-coloração qualquer das arestas da estrela $K^{1, m+n-1}$. Para provar que $\hat{r}\left(K^{1, n}, K^{1, m}\right) \geqslant m+n-1$, vamos mostrar o seguinte: se $H$ é um grafo com no máximo $m+n-2$ arestas, então $E(H)$ pode ser colorido de vermelho e azul sem que ocorra nenhuma cópia vermelha do $K^{1, n}$ e nem cópia do azul $K^{1, m}$.

De fato, vamos supor que $n \geqslant m$. Se o grau máximo em $H$ é menor que $n$, então basta colorir todas as arestas de vermelho. Assim, podemos supor que existe um vértice $v$ em $H$ de grau pelo menos $n$. Mas, $e(H) \leqslant m+n-2$ implica que esse vértice é único e que o grau máximo em $H-v$ é menor que $m$. Assim, qualquer 2-coloração de $E(H-v)$ não cria cópias monocromáticas de nenhuma das estrelas. Escolhemos, arbitrariamente, uma dessas 2-colorações e a estendemos para todo $H$ colorindo as arestas que incidem em $v$ de maneira a não criar estrelas monocromáticas.

Dado um grafo $H$, denotamos por $H^{(s)}$ o grafo obtido a partir de $H$ substituindo cada aresta $x y \in E(H)$ por um $x-y$ caminho de comprimento $s \in \mathbb{N}$, sendo que arestas distintas 
dão origem a caminhos disjuntos nos vértices internos. Chamamos $H^{(s)}$ de uma subdivisão de H. O nosso resultado (Donadelli e Kohayakawa, 2002) no Capítulo 4 é o seguinte:

Seja $H$ um grafo dado. Existem constantes $C_{1}$ e $C_{2}$ e existe um grafo $\Gamma^{n}$, para todo $n$ suficientemente grande, com $O(n)$ arestas tal que $G^{n} \rightarrow\left(H^{(s)}, H^{(s)}\right)$ para todo $C_{1} \log n \leqslant s \leqslant$ $C_{2} n$.

Para demonstrar o resultado enunciado acima, também fazemos uso do Lema de Regularidade, mas dessa vez sem a técnica de Füredi. Uma outra ferramenta que usamos é um resultado de Pósa (1976) que diz que grafos que são bons expansores contêm caminhos longos. Dito isso, a nossa técnica é mostrar que pares regulares são bons expansores e usar os pares dados pelo Lema de Regularidade para encontrar uma subdivisão do grafo $H$ contida num grafo pseudo-aleatório $G^{n} \operatorname{com} O(n)$ arestas.

Finalmente, apresentamos de forma sistematizada as notações empregadas na presente tese. Esperamos que, com essa introdução às notações, tenhamos coberto tudo o que está sendo usado neste trabalho. Caso o leitor encontre situações não definidas, sugerimos que consulte Diestel (1997) e/ou Janson et al. (2000).

\section{Teoria dos Grafos}

Como já é usual em combinatória, denotamos por $[n]$ o subconjunto

$$
[n]=\{1,2, \ldots, n\} \subseteq \mathbb{N},
$$

onde $\mathbb{N}$ é o conjunto dos números naturais. Também é usual a seguinte notação: se $V$ é um conjunto finito qualquer e $0 \leqslant k \leqslant|V|$, então usamos $\left(\begin{array}{l}V \\ k\end{array}\right)$ para denotar a família de todos os subconjuntos de $V$ com cardinalidade $k$, ou seja,

$$
\left(\begin{array}{l}
V \\
k
\end{array}\right)=\{U: U \subseteq V \text { e }|U|=k\}
$$


Usamos $2^{V}$ para denotar o conjunto de todos os subconjuntos de $V$, logo

$$
2^{V}=\bigcup_{k=0}^{|V|}\left(\begin{array}{l}
V \\
k
\end{array}\right) \text {. }
$$

Um hipergrafo $G$ é um par ordenado $G=(V, E)$, onde $V=V(G)$, é um conjunto finito cujos elementos são chamados vértices e $E=E(G) \subseteq 2^{V}$ é o conjunto das arestas de $G$. Quando todas as arestas de $G$ têm a mesma cardinalidade, digamos que $E \subseteq\left(\begin{array}{l}V \\ k\end{array}\right)$, então chamamos $G$ de hipergrafo k-uniforme. Quando quaisquer duas arestas de $G$ têm no máximo um elemento comum chamamos $G$ de hipergrafo linear.

Num hipergrafo $G$, um hipercircuito, ou simplesmente circuito, é definido por uma seqüência $v_{1}, E_{1}, v_{2}, E_{2} \ldots, v_{r}, E_{r}$, para $r \geqslant 2$, onde os vértices são dois-a-dois distintos, as arestas $E_{i}$ são duas-a-duas distintas e $\left\{v_{i}, v_{i+1}\right\} \subseteq E_{i}$, para todo $i \in[r-1]$, e $\left\{v_{1}, v_{r}\right\} \subseteq E_{r}$. Um hipercircuito com $r$ vértices e $r$ arestas é denotado por $\mathcal{C}^{r}$.

Um grafo é um hipergrafo 2-uniforme. Note que essa definição de grafo exclui os grafos com laços e com arestas múltiplas. Nas próximas definições estamos nos referindo a grafos; não é difícil estender estas definições para hipergrafos.

Usamos $v(G)$ para denotar $|V(G)|$, o número de vértices em $G$, e usamos $e(G)$ para denotar $|E(G)|$, o número de arestas em $G$. Dado um vértice $v \in V(G)$, chamamos de vizinhança de $v$ o conjunto

$$
\Gamma_{G}(v)=\{w \in V(G):\{v, w\} \in E(G)\},
$$

cuja cardinalidade denotamos por $d_{G}(v)$ e chamamos de grau de $v$ em $G$.

Dizemos que $H$ é um subgrafo de $G$, e escrevemos $H \subseteq G$, se $H$ é um grafo tal que $V(H) \subseteq$ $V(G)$ e $E(H) \subseteq E(G)$. Um subgrafo $H$ é dito subgrafo induzido se $E(H)=E(G) \cap\left(\begin{array}{c}V(H) \\ 2\end{array}\right)$. Ademais, se $U \subseteq V(G)$, então $U$ define naturalmente um subgrafo induzido, que denotamos por $G[U]$; também, se $F \subseteq E(G)$ então $G[F]=G[U]$, onde $U=\bigcup_{e \in F} e$.

Dois grafos $G$ e $H$ são isomorfos quando existe uma bijeção $\iota: V(G) \rightarrow V(H)$, tal que $\{v, w\} \in E(G)$ se, e somente se, $\{\iota(v), \iota(w)\} \in E(H)$. Por uma cópia de $H$ em $G$ entendemos um subgrafo $\bar{H} \subseteq G$ que é isomorfo a $H$.

Um caminho em $G$ é uma seqüência $v_{1}, v_{2}, \ldots, v_{r-1}, v_{r}$ de vértices $v_{i} \in V(G)$ dois-a-dois distintos tais que $\left\{v_{i}, v_{i+1}\right\} \in E(G)$, para todo $i \in[r-1]$. O grafo definido pelo caminho com $r$ 
vértices e $r-1$ arestas é denotado por $P^{r}$. Um circuito em $G$ é um caminho $v_{1}, v_{2}, \ldots, v_{r}$ com a condição adicional que $\left\{v_{1}, v_{r}\right\} \in E(G)$. O circuito com $r$ vértices e $r$ arestas é denotado por $C^{r}$. Uma aresta que liga dois vértices não consecutivos de um circuito $C^{r}(r \geqslant 4)$ é chamada de corda. A cintura de um grafo é o mínimo entre os comprimentos dos circuitos desse grafo.

Dizemos que um grafo $G$ é conexo se quaisquer dois vértices do grafo pertencem a um mesmo caminho em $G$. Uma árvore é um grafo conexo sem circuitos. Uma floresta é uma união disjunta de árvores e um emparelhamento é uma floresta onde as arestas são duas-a-duas disjuntas. Um grafo conexo $G$ é 2-conexo se ele tem pelo menos três vértices e a remoção de quaisquer dois vértices não desconecta o grafo.

O grafo com $r$ vértices, $r \in \mathbb{N}$, e todas as $\left(\begin{array}{c}r \\ 2\end{array}\right)$ arestas é denotado por $K^{r}$ e chamado de grafo completo. Por $K^{r, s}$ denotamos o grafo bipartido completo com $r$ vértices numa partição e $s$ vértices noutra sendo as únicas arestas as $r s$ arestas que ligam vértices de partições distintas. Quando $r=1$, o grafo bipartido completo é chamado estrela.

Seja $r \geqslant 1$ um inteiro qualquer e consideremos duas funções quaisquer $\phi: V(G) \rightarrow[r]$ e $\varphi: E(G) \rightarrow[r]$. Chamamos essas funções de $r$-coloração dos vértices e das arestas, respectivamente, de $G$. Também, chamamos $\phi$ de coloração própria dos vértices de $G$, se para toda aresta $e=\{u, v\} \in E(G)$ temos $|\phi(e)|>1$, ou seja, se sob a coloração $\phi$ não existem arestas monocromáticas. O menor $r \in \mathbb{N}$ para o qual existe uma $r$-coloração própria dos vértices de $G$ é chamado o número cromático de $G$, sendo denotado por $\chi(G)$.

De um modo geral, escrevemos $G=G^{n}$ quando queremos indicar que o grafo $G$ tem $n$ vértices.

\section{Teorias de Ramsey e Extremal}

Dados os grafos $\Gamma, G_{1}, \ldots, G_{q}(q \geqslant 1)$, escrevemos $\Gamma \rightarrow\left(G_{1}, \ldots, G_{q}\right)$ se, para qualquer $q$-coloração $\varphi: E(\Gamma) \rightarrow[q]$ das arestas de $\Gamma$, existe $i \in[q]$ tal que

$$
\Gamma\left[\varphi^{-1}(i)\right] \text { contém uma cópia de } G_{i}
$$


ou seja, existe $i \in[q]$ tal que o subgrafo de $\Gamma$ induzido pelas arestas da cor $i$ contém um subgrafo $H$ isomorfo ao grafo $G_{i}$. Neste caso, dizemos que $\Gamma$ é ramsey com relação à $q$-upla $\left(G_{1}, \ldots, G_{q}\right)$. Definimos o número de ramsey

$$
r\left(G_{1}, \ldots, G_{q}\right)=\min \left\{n: K^{n} \rightarrow\left(G_{1}, \ldots, G_{q}\right)\right\}
$$

O Teorema de Ramsey garante que existe $n \in \mathbb{N}$ tal que $K^{n} \rightarrow\left(G_{1}, \ldots, G_{q}\right)$. O resultado abaixo generaliza esse, e diz que se $n$ é suficientemente grande, então o número de cópias monocromáticas é polinomial em $n$.

Lema 5. Seja $q \geqslant 1$ um inteiro. Dados os grafos $G_{1}, \ldots, G_{q}$, existem constantes positivas $c=c\left(G_{1}, \ldots, G_{q}\right)>0$ e $k_{0}=k_{0}\left(G_{1}, \ldots, G_{q}\right)$ tais que, para todo $k \geqslant k_{0}$, se as arestas do $K^{k}$ são $q$-coloridas arbitrariamente, então para algum $i \in[q]$ ocorrem pelo menos ck $^{v\left(G_{i}\right)}$ cópias monocromáticas de $G_{i}$ da cor $i$.

De fato, basta tomar $k_{0}=r\left(G_{1}, \ldots, G_{q}\right)$ e $c=(1 / q) k_{0}-\max _{j} v\left(G_{j}\right)$. Consideramos o grafo completo $K^{k}$ com $k \geqslant k_{0}$ vértices e com uma $q$ coloração arbitrária das arestas. Agora, pela definição de $k_{0}$, cada subconjunto de $V\left(K^{k}\right)$ com cardinalidade $k_{0}$ contém uma cópia de $G_{i}$ monocromática da cor $i$, para algum $i \in[q]$.

Dentre as $q$ cores temos que $\frac{1}{q}\left(\begin{array}{c}k \\ k_{0}\end{array}\right)$ daqueles $k_{0}$-subconjuntos de vértices apresentam cópias de $G_{i}$ monocromáticas da cor $i$, para algum $i \in[q]$. Cada uma dessas cópias é contada no máximo $\left(\begin{array}{c}k-v\left(G_{i}\right) \\ k_{0}-v\left(G_{i}\right)\end{array}\right)$ vezes, portanto, o número de tais cópias é pelo menos

$$
\frac{1}{q} \frac{\left(\begin{array}{c}
k \\
k_{0}
\end{array}\right)}{\left(\begin{array}{c}
k-v\left(G_{i}\right) \\
k_{0}-v\left(G_{i}\right)
\end{array}\right)}=\frac{1}{q} \frac{k(k-1) \cdots\left(k-v\left(G_{i}\right)+1\right)}{k_{0}\left(k_{0}-1\right) \cdots\left(k_{0}-v\left(G_{i}\right)+1\right)} \geqslant \frac{1}{q}\left(\frac{k}{k_{0}}\right)^{v\left(G_{i}\right)} \geqslant c k^{v\left(G_{i}\right)} .
$$

Definimos por $\operatorname{ex}(G, H)$ o máximo de arestas que um subgrafo de $G$ pode ter para não conter uma cópia de $H$, ou seja

$$
\operatorname{ex}(G, H)=\max \{e(F): H \nsubseteq F \subseteq G\}
$$

e, em particular, $\operatorname{ex}(n, H)=\operatorname{ex}\left(K^{n}, H\right)$. Por exemplo, para toda floresta $F$ com $t$ vértices, vale que

$$
\operatorname{ex}(G, F)<(t-1) v(G)
$$


Em 1941, Turán (veja Diestel, 1997, Teorema 7.1.1) provou que o grafo $(t-1)$-partido com $n$ vértices distribuídos o mais igualmente possível dentre as $t-1$ classes, o qual denotamos por $T^{t-1}(n)$, é extremal com relação a conter $K^{t}$, ou seja, é um grafo com $n$ vértices onde a adição de qualquer nova aresta cria uma cópia do $K^{t}$, quaisquer que sejam $n, t \geqslant 1$. Mais que isso, esse grafo é único com essa propriedade.

Teorema 6. Para todos $n, t>1$ vale que ex $\left(n, K^{t}\right)=e\left(T^{t-1}(n)\right)$. Ainda, todo grafo $G$ de ordem $n$ que não contém $K^{t}$ e com $\operatorname{ex}\left(n, K^{t}\right)$ arestas é isomorfo ao grafo $T^{t-1}(n)$.

Logo, todo grafo $G^{n}$ com

$$
e\left(G^{n}\right)>\left(1-\frac{1}{t-1}\right) \frac{n^{2}}{2}
$$

contém uma cópia do $K^{t}$.

Um resultado interessantíssimo de Erdös e Stone (1946) diz que

$$
\lim _{n \rightarrow \infty} \frac{\operatorname{ex}(n, H)}{\left(\begin{array}{l}
n \\
2
\end{array}\right)}=1-\frac{1}{\chi(H)-1}
$$

Em particular, no caso de circuitos, para todo $\varepsilon$ positivo e $n$ suficientemente grande

$$
\operatorname{ex}\left(n, C^{\ell}\right)< \begin{cases}\varepsilon n^{2} & \text { se } \ell \text { é par } \\
(1 / 2+\varepsilon)\left(\begin{array}{l}
n \\
2
\end{array}\right) & \text { se } \ell \text { é ímpar. }\end{cases}
$$

\section{Notação assintótica}

Sejam $f_{n}$ e $g_{n}$ seqüências de números reais, onde $f_{n}>0$ para todo $n$ suficientemente grande. Usaremos as seguintes notações para o comportamento assintótico dessas seqüências:

- $g_{n}=O\left(f_{n}\right)$, quando $n \rightarrow \infty$, se existem constantes positivas $c \in \mathbb{R}$ e $n_{0} \in \mathbb{N}$ tais que $\left|g_{n}\right| \leqslant c f_{n}$, para todo $n \geqslant n_{0}$

- $g_{n}=\Omega\left(f_{n}\right)$, quando $n \rightarrow \infty$, se existem constantes positivas $C \in \mathbb{R}$ e $n_{0} \in \mathbb{N}$ tais que $g_{n} \geqslant C f_{n}$, para todo $n \geqslant n_{0}$

- $g_{n}=\Theta\left(f_{n}\right)$, quando $n \rightarrow \infty$, se existem constantes positivas $c, C \in \mathbb{R}$ e $n_{0} \in \mathbb{N}$ tais que $C f_{n} \leqslant g_{n} \leqslant c f_{n}$, para todo $n \geqslant n_{0}$; 
- $g_{n} \asymp f_{n}$, quando $n \rightarrow \infty$, se existem constantes positivas $C \in \mathbb{R}$ e $n_{0} \in \mathbb{N}$ tais que $(1 / C) f_{n} \leqslant g_{n} \leqslant C f_{n}$, para todo $n \geqslant n_{0}$;

- $g_{n}=o\left(f_{n}\right)$ se $g_{n} / f_{n} \rightarrow 0$, quando $n \rightarrow \infty$.

\section{Probabilidade discreta}

Sejam $\omega_{1}, \omega_{2}, \ldots, \omega_{N}$ todos os possíveis resultados de um experimento idealizado. Chamamos $\omega_{1}, \omega_{2}, \ldots, \omega_{N}$ de eventos elementares e o conjunto finito $\Omega=\left\{\omega_{1}, \omega_{2}, \ldots, \omega_{N}\right\}$ é dito espaço amostral, ou seja, o conjunto de todos os possíveis resultados do experimento. Um subconjunto $A \subseteq \Omega$ para o qual, sob as condições do experimento, é possivel dizer ou que "o resultado $\omega$ pertence a $A$ ", ou que "o resultado $\omega$ não pertence a $A$ " é chamado de evento.

Agora, definimos um "peso" para cada evento elementar. Tal peso é uma função qualquer definida em $\Omega$ e que assume valores no intervalo fechado $[0,1] \subset \mathbb{R}$, isto é $\mathbb{P}: \Omega \rightarrow[0,1]$, e tal que $\mathbb{P}\left(\omega_{1}\right)+\cdots+\mathbb{P}\left(\omega_{N}\right)=1$.

O par $(\Omega, \mathbb{P})$ é chamado de de espaço de probabilidade ${ }^{1}$, onde $\Omega$ é o espaço amostral e $\mathbb{P}$ é dita função de probabilidade. Ademais, definimos $\mathbb{P}(A)=\sum_{a \in A} \mathbb{P}(a)$ para todo evento $A \subseteq \Omega$.

Para nós, uma variável aleatória $X$, num espaço de probabilidade $(\Omega, \mathbb{P})$, é uma função $X: \Omega \rightarrow \mathbb{R}$. Escrevemos $\mathbb{P}\{X=t\}$ para $\mathbb{P}\left(A_{t}\right)$, onde $A_{t} \subseteq \Omega$ é o conjunto de todos os pontos $\omega \in \Omega$ tais que $X(\omega)=t$, ou seja,

$$
\mathbb{P}\{X=t\}=\mathbb{P}(\{\omega \in \Omega: X(\omega)=t\})
$$

Definimos $\mathbb{P}\{X \geqslant t\}$ analogamente.

\footnotetext{
${ }^{1}$ Estritamente falando, um espaço de probabilidades é uma tripla $(\Omega, \mathcal{A}, \mathbb{P})$, onde $\Omega$ é um conjunto qualquer e $\mathbb{P}$ é uma medida aditiva sobre $\mathcal{A}$ tal que $\mathbb{P}(\mathcal{A})=1$. Ademais, $\mathcal{A} \subseteq 2^{\Omega}$ é uma coleção de subconjuntos de $\Omega$ contendo $\Omega$ e fechada sob complementação, união enumerável e intersecção enumerável; esses são os eventos. Para nós, sempre $\mathcal{A}=2^{\Omega}$ nos modelos probabilísticos. Além disso, $\Omega$ é finito, portanto $\mathbb{P}$ é determinada pelos seus valores nos elementos de $\Omega$.
} 
O valor esperado da variável aleatória $X$, que denotamos por $\mathbb{E} X$, é dado pela média

$$
\mathbb{E} X=\sum_{t \in X(\Omega)} t \mathbb{P}\{X=t\} .
$$

Daqui por diante, será importante termos em mente que estaremos tratando com resultados assintóticos. Sendo mais preciso, estaremos diante de situações como a que segue. Seja $\left\{\left(\Omega_{n}, \mathbb{P}_{n}\right)\right\}_{n \in \mathbb{N}}$ uma seqüência de espaços de probabilidade. Suponha que temos uma seqüência de eventos $A_{n} \subseteq \Omega_{n}$. Então, estaremos interessados em saber resultados como,

$$
\mathbb{P}_{n}\left(A_{n}\right)>1 / 2 \text {, para todo } n \text { suficientemente grande, }
$$

ou

$$
\lim _{n \rightarrow \infty} \mathbb{P}_{n}\left(A_{n}\right)=1 .
$$

Também, se $\left\{A_{n}\right\}_{n \in \mathbb{N}}$ é uma seqüência de eventos $A_{n} \subseteq \Omega_{n}$ de modo que $\mathbb{P}_{n}\left(A_{n}\right) \rightarrow 1$ quando $n \rightarrow \infty$, dizemos que ocorre $A_{n}$ quase certamente.

Desigualdades, desvios e momentos. Para todo inteiro positivo $k$, o $k$-ésimo momento de uma variável aleatória $X$ é o valor esperado de sua $k$-ésima potência $\mathbb{E} X^{k}$.

O método do primeiro momento é a ferramenta mais simples no uso da esperança e segue facilmente da sua definição:

$$
\mathbb{E} X \leqslant t \Rightarrow \mathbb{P}\{X \leqslant t\}>0 .
$$

Note que se $X \geqslant 0$ e $t_{0}>0$, então

$$
\mathbb{E} X=\sum_{t \in X(\Omega)} t \mathbb{P}\{X=t\} \geqslant t_{0} \sum_{\substack{t \in X(\Omega) \\ t \geqslant t_{0}}} \mathbb{P}\{X=t\}=t_{0} \mathbb{P}\left\{X \geqslant t_{0}\right\},
$$

donde concluímos a seguinte desigualdade.

Desigualdade De Markov. Se $X$ é uma variável aleatória positiva e $t>0$ então

$$
\mathbb{P}\{X \geqslant t\} \leqslant \frac{\mathbb{E} X}{t} .
$$

Note que, se $\left\{X_{n}\right\}_{n \in \mathbb{N}}$ é uma seqüência de variáveis aleatórias definidas sobre $\left\{\left(\Omega_{n}, \mathbb{P}_{n}\right)\right\}_{n \in \mathbb{N}}$ de modo que $\mathbb{E} X_{n} \rightarrow 0$, quando $n \rightarrow \infty$, então nós podemos deduzir de (5) que $\mathbb{P}\left\{X_{n}>0\right\} \rightarrow$ 0 quando $n \rightarrow \infty$ e, nesse caso, temos $X_{n}=0$ quase certamente. 
Agora, se queremos mostrar que a probabilidade $\mathbb{P}\{X \geqslant t\}$ é alta, não é suficiente limitar inferiormente $\mathbb{E} X$. Podemos ter que o valor esperado de $X$ pode ser grande, simplesmente porque $X$ assume valores muito grandes em poucos elementos de $\Omega$, e pode até ser que $X<t$ para a maior parte dos pontos de $\Omega$. Assim, para provar que $\mathbb{P}\{X \geqslant t\}$ é grande, temos que mostrar que $X$ não desvia freqüentemente do seu valor esperado. O modo mais elementar de fazermos isso é o seguinte. A variância de $X$ é dada por

$$
\operatorname{Var}(X)=\mathbb{E}(X-\mathbb{E} X)^{2}=\mathbb{E} X^{2}-(\mathbb{E} X)^{2}
$$

Dessa forma, se $\lambda$ é um real positivo, então

$$
\mathbb{P}\{|X-\mathbb{E} X| \geqslant \lambda \sqrt{\operatorname{Var}(X)}\}=\mathbb{P}\left\{(X-\mathbb{E} X)^{2} \geqslant \lambda^{2} \operatorname{Var}(X)\right\}
$$

Usando a desigualdade de Markov e fazendo a escolha apropriada para $\lambda$, temos um método de segundo momento.

Desigualdade De Chebyshev. Para todo real positivo $\varepsilon$ e variável aleatória $X$ temos

$$
\mathbb{P}\{|X-\mathbb{E} X| \geqslant \varepsilon \mathbb{E} X\} \leqslant \frac{\operatorname{Var}(X)}{\varepsilon^{2}(\mathbb{E} X)^{2}} .
$$

Da desigualdade acima, podemos facilmente concluir que se $\operatorname{Var}(X)=o\left((\mathbb{E} X)^{2}\right)$, então $X>0$ quase certamente, ou ainda, $X \asymp \mathbb{E} X$ quase certamente.

Voltemos à nossa seqüência de espaços de probabilidade $\left\{\left(\Omega_{n}, \mathbb{P}_{n}\right)\right\}_{n \in \mathbb{N}}$. Muitas vezes quando usamos a Desigualdade de Chebyshev conseguimos provar que a probabilidade de que um evento falhe tende a zero polinomialmente. Ocorre que, quando queremos usar essa probabilidade um número exponencial de vezes, a Desigualdade de Chebyshev não nos dá informação suficiente e temos que recorrer às desigualdades exponenciais.

Usamos $\operatorname{Bi}(n, p)$ para denotar a distribuição binomial. 
Desigualdade de Chernoff (Janson et al., 2000). Se $X \in \operatorname{Bi}(n, p), \lambda \geqslant 0$ e $\varphi(x)=$ $(1+x) \ln (1+x)-x$, para todo $x \geqslant-1$, então

$$
\mathbb{P}\{X-\mathbb{E} X \geqslant \lambda\} \leqslant \exp \left\{-\mathbb{E} X \varphi\left(\frac{\lambda}{\mathbb{E} X}\right)\right\} \leqslant \exp \left\{-\frac{\lambda^{2}}{2(\mathbb{E} X+\lambda / 3)}\right\}
$$

$$
\mathbb{P}\{X-\mathbb{E} X \leqslant-\lambda\} \leqslant \exp \left\{-\mathbb{E} X \varphi\left(\frac{\lambda}{\mathbb{E} X}\right)\right\} \leqslant \exp \left\{-\frac{\lambda^{2}}{2 \mathbb{E} X}\right\}
$$

Da equação acima deduzimos que para todo $\lambda>0$

$$
\mathbb{P}\{|X-\mathbb{E} X| \geqslant \lambda \mathbb{E} X\} \leqslant 2 \exp \{-\varphi(\lambda) \mathbb{E} X\} \leqslant 2 \exp \left\{-c_{\lambda} \mathbb{E} X\right\},
$$

onde $c_{\lambda}=3 \lambda^{2} /(6+2 \lambda)$.

\section{Modelos de grafos aleatórios}

Vamos denotar por $\mathcal{G}(n)$ o conjunto de todos os grafos sobre o conjunto de vértices $V=$ $[n]=\{1,2, \ldots, n\}$.

Vejamos os dois modelos mais conhecidos para grafos aleatórios.

Grafo aleatório binomial. O primeiro modelo é denotado por $\mathcal{G}(n, p)$, onde $0 \leqslant p \leqslant 1$. Nesse modelo o espaço amostral é o conjunto de todos os resultados possíveis do seguinte experimento. Dado $p$, com $0 \leqslant p \leqslant 1$, considere uma moeda com probabilidade $p$ de dar cara. Começamos com o grafo sem arestas, isto é, o grafo $([n], \emptyset)$ e, para cada $\{i, j\} \in\left(\begin{array}{c}{[n]} \\ 2\end{array}\right)$, lançamos a nossa moeda independentemente para cada par de vértices. Se o resultado for cara colocamos a aresta $\{i, j\}$ no grafo, caso contrário, não colocamos a aresta. Um grafo genérico definido desta forma é denotado por $G_{n, p}$ e o chamamos de grafo aleatório com $n$ vértices e probabilidade de arestas $p$.

Equivalentemente, temos $\mathcal{G}(n, p)$ considerando o conjunto $\mathcal{G}(n)$ com a função de probabilidade

$$
\mathbb{P}_{p}(J)=p^{e}(1-p)^{\left(\begin{array}{l}
n \\
2
\end{array}\right)-e},
$$

para um grafo $J \in \mathcal{G}(n)$ com $e$ arestas. 
Grafo aleatório uniforme. O outro modelo para grafos aleatórios, denotado por $\mathcal{G}(n, M)$, tem como espaço amostral o conjunto de todos os $\left(\begin{array}{l}N \\ M\end{array}\right)$ grafos sobre $V=[n]$ que têm exatamente $M$ arestas, onde $0 \leqslant M \leqslant N=\left(\begin{array}{c}n \\ 2\end{array}\right)$. Tomamos sobre esse espaço a distribuição uniforme, ou seja, se $J \in \mathcal{G}(n)$ então

$$
\mathbb{P}_{M}(J)=\left(\begin{array}{l}
N \\
M
\end{array}\right)^{-1}
$$

Escrevemos $G_{n, M}$ para um grafo genérico sorteado uniformemente dentre todos os grafos com $n$ vértices e $M$ arestas.

Advertência: A próxima seção contém informações que não serão usadas. Resolvemos colocá-las aqui porque podem ser úteis ao entendimento de alguns resultados deste trabalho.

Equivalências dos modelos de grafos aleatórios. Seja $\mathcal{Q}$ uma família de grafos. Pela Lei das Probabilidades Totais temos

$$
\mathbb{P}\left\{G_{n, p} \in \mathcal{Q}\right\}=\sum_{M=0}^{\left(\begin{array}{c}
n \\
2
\end{array}\right)} \mathbb{P}\left\{G_{n, M} \in \mathcal{Q}\right\}\left(\begin{array}{c}
\left(\begin{array}{c}
n \\
2
\end{array}\right) \\
M
\end{array}\right) p^{M}(1-p)^{\left(\begin{array}{c}
n \\
2
\end{array}\right)-M} .
$$

Dizemos que $\mathcal{Q}$ é uma propriedade crescente se todo grafo que contém algum grafo de $\mathcal{Q}$ também pertence a $\mathcal{Q}$, isto é, $H \subseteq G$ e $H \in \mathcal{Q} \Rightarrow G \in \mathcal{Q}$.

Teorema 7. Seja $\mathcal{Q}$ crescente, $M=M(n) \rightarrow \infty$ e $\delta>0$ fixo tal que $0 \leqslant(1-\delta) p<$ $(1+\delta) p \leqslant 1$, onde $p=M /\left(\begin{array}{l}n \\ 2\end{array}\right)$.

1. Se $\mathbb{P}\left\{G_{n, p} \in \mathcal{Q}\right\} \rightarrow 1$, então $\mathbb{P}\left\{G_{n, M} \in \mathcal{Q}\right\} \rightarrow 1$.

2. Se $\mathbb{P}\left\{G_{n, p} \in \mathcal{Q}\right\} \rightarrow 0$, então $\mathbb{P}\left\{G_{n, M} \in \mathcal{Q}\right\} \rightarrow 0$.

3. Se $\mathbb{P}\left\{G_{n, M} \in \mathcal{Q}\right\} \rightarrow 1$, então $\mathbb{P}\left\{G_{n,(1+\delta) p} \in \mathcal{Q}\right\} \rightarrow 1$.

4. Se $\mathbb{P}\left\{G_{n, M} \in \mathcal{Q}\right\} \rightarrow 0$, então $\mathbb{P}\left\{G_{n,(1-\delta) p} \in \mathcal{Q}\right\} \rightarrow 0$. 


\section{CAPíTULO 0}

\section{O LEMA DE REGULARIDADE DE SZEMERÉDI}

O Lema de Regularidade foi usado por Szemerédi como resultado auxiliar na prova do Teorema 2, página 17, e tornou-se uma ferramenta de grande importância na Teoria dos Grafos contemporânea.

Ingenuamente, o Lema de Szemerédi diz que o conjunto dos vértices de todo grafo pode ser particionado em um pequeno número de subconjuntos com cardinalidades que diferem de no máximo um, de forma que muitos dos subgrafos bipartidos induzidos têm suas arestas distribuídas de maneira regular, quase uniforme. Esses subgrafos satisfazem várias propriedades locais de grafos bipartidos aleatórios. Como exemplo, veja a Afirmação 1 na página 39 que afirma que muitos vértices desses grafos bipartidos pseudo-aleatórios têm graus "próximos". Conquanto grafos aleatórios de uma certa densidade de arestas são mais fáceis de serem manipulados que a classe de todos os grafos da mesma densidade, usamos o Lema de Regularidade para transportar resultados que são "fáceis" em grafos aleatórios para a classe dos grafos daquela densidade.

\section{Introdução}

Sejam $G=(V, E)$ um grafo e $U, W \subseteq V$ subconjuntos disjuntos de $V$. Então, denotamos por $e_{G}(U, W)$ o número de arestas, em $G$, com um extremo em $U$ e outro em $W$, sendo

$$
d_{G}(U, W)=\frac{e_{G}(U, W)}{|U||W|}
$$

a densidade do par $(U, W)$ no grafo $G$.

Dado $0<\varepsilon<1$, dizemos que o par $(U, W)$ é $(\varepsilon, G)$-regular, se para quaisquer $X \subseteq U$ e $Y \subseteq W \operatorname{com}|X| \geqslant \varepsilon|U|$ e $|Y| \geqslant \varepsilon|W|$ vale que

$$
\left|d_{G}(X, Y)-d_{G}(U, W)\right|<\varepsilon .
$$


Observe que, mais uniforme é a distribuição das arestas no par $(U, W)$, quanto menor for o valor de $\varepsilon$.

Quando o grafo $G$ estiver subentendido e não houver perigo de confusão, usamos $e(U, W)$ para denotar $e_{G}(U, W)$, escrevemos $d(U, W)$ para a densidade $d_{G}(U, W)$ e chamamos um par $(\varepsilon, G)$-regular de $\varepsilon$-regular.

Exemplo 1. O grafo bipartido aleatório definido sobre $[n] \times[n]$ cujas arestas são escolhidas independentemente com probabilidade $p \in[0,1]$ é $\varepsilon$-regular com probabilidade tendendo a 1 , como mostra uma aplicação direta da desigualdade de Chernoff, equação (8) na página 33.

Observação 2. Se $d(U, W) \leqslant \varepsilon^{3}$, então $U$ e $W$ formam um par $\varepsilon$-regular, ou seja, pares muito esparsos são necessariamente regulares. De fato, se $X \subseteq U$ com $|X| \geqslant \varepsilon|U|$ e $Y \subseteq W$ com $|Y| \geqslant \varepsilon|W|$, então

$$
d(X, Y)=\frac{e(X, Y)}{|X||Y|}<\frac{e(U, W)}{|X||Y|} \leqslant \varepsilon^{3} \frac{|U||W|}{|X||Y|} \leqslant \varepsilon .
$$

Agora, se $U$ e $W$ formam um par $\varepsilon$-regular em $G$, então eles também formam um par $\varepsilon$-regular no complemento de $G$, logo, podemos concluir que pares densos também são necessariamente regulares.

Uma $(\varepsilon, k, G)$-eqüipartição é uma partição do conjunto de vértices de $G=(V, E)$ em $k+1$ partes $V_{0}, V_{1}, \ldots, V_{k}$ tal que $\left|V_{0}\right| \leqslant \varepsilon|V|$ e $V_{0}$ é chamado conjunto excepcional, e $\left|V_{i}\right|=\left|V_{j}\right|$ para todos $1 \leq i<j \leq k$. Chamamos uma partição de $(\varepsilon, k, G)$-regular, se essa for uma $(\varepsilon, k, G)$ eqüipartição onde o número de pares $\left(V_{i}, V_{j}\right)$ que não são $\varepsilon$-regulares é no máximo $\varepsilon\left(\begin{array}{l}k \\ 2\end{array}\right)$, para $1 \leq i<j \leq k$.

O Lema de Regularidade de Szemerédi é o seguinte resultado de 1978.

TeOrema 8 (Szemerédi 1978). Dados um real $0<\varepsilon<1$ e um inteiro $k_{0} \geq 1$, existem inteiros positivos $n_{0}=n_{0}\left(\varepsilon, k_{0}\right)$ e $K_{0}=K_{0}\left(\varepsilon, k_{0}\right) \geqslant k_{0}$ tais que qualquer grafo $G$ com pelo menos $n_{0}$ vértices admite uma partição $(\varepsilon, k, G)$-regular para algum $k_{0} \leq k \leq K_{0}$.

O papel do conjunto excepcional no Lema de Regularidade é garantir que as outras classes tenham exatamente a mesma cardinalidade. Não é difícil ver que, nas condições do lema acima, existe uma partição $\Pi=\left(V_{1}, \ldots, V_{k}\right)$ de $V$ tal que ||$V_{i}|-| V_{j}|| \leqslant 1$ e o número de pares $\left(V_{i}, V_{j}\right)$ que não são $\varepsilon$-regulares é no máximo $\varepsilon\left(\begin{array}{c}k \\ 2\end{array}\right)$, para todos $1 \leqslant i<j \leqslant k$ e algum $k_{0} \leq k \leq K_{0}$. 
De fato, podemos distribuir igualmente os vértices de $V_{0}$ entre as outras classes, de modo que a $\varepsilon$-regularidade é preservada para um $\varepsilon$ ligeiramente maior. Mais especificamente, dados $\varepsilon$ e $k_{0}$, aplicamos o Teorema 8 para $\varepsilon^{2} / 8$ e $k_{0}$. Tomamos $\Pi^{\prime}$ a partição que é dada pelo lema e definimos uma nova partição $\Pi$, a partir de $\Pi^{\prime}$, dividindo o mais igualmente possível os, no máximo $\varepsilon^{2} n / 8$, vértices de $V_{0}^{\prime} \in \Pi^{\prime}$ entre as classes não-excepcionais de $\Pi^{\prime}$. Tomamos $n_{0}$ e $K_{0}$ e mostramos que se $\left(V_{l}^{\prime}, V_{j}^{\prime}\right)$ é um par $\varepsilon^{2} / 8$-regular de $\Pi^{\prime}$, então o par $\left(V_{l}, V_{j}\right)$, na nova partição $\Pi$, é $\varepsilon$-regular, onde $V_{l}$ é obtido de $V_{l}^{\prime}$ quando distribuímos os vértices da classe excepcional de $\Pi^{\prime}$. Como não usaremos essa versão do lema, não vamos detalhar esse esboço de prova que acabamos de descrever.

Vejamos como podemos aplicar o lema. Dados $0<\varepsilon<1$ real e $k_{0} \geqslant 1$ inteiro, apliquemos o Lema de Regularidade de Szemerédi para um grafo $G$ de ordem $n$ suficientemente grande. Observe que

$$
(1-\varepsilon) \frac{n}{k} \leqslant\left|V_{i}\right| \leqslant \frac{n}{k}
$$

para todo $i \in[k]$. Ainda, dado um real positivo $\rho<1$, considere os pares $\left(V_{i}, V_{j}\right)$, para $1 \leqslant$ $i<j \leqslant k$, que são $\varepsilon$-regulares com densidade pelo menos $\rho$. Então, pondo $\left|V_{i}\right|=m$ para todo $i \in[k]$, temos que

(i) $V_{0}$ contém no máximo $1 / 2(\varepsilon n)^{2}$ arestas e existem no máximo $\left|V_{0}\right| m k \leqslant \varepsilon n m k$ arestas que ligam vértices de $V_{0}$ aos vértices das outras classes;

(ii) cada um dos no máximo $\varepsilon\left(\begin{array}{c}k \\ 2\end{array}\right)$ pares irregulares contém no máximo $m^{2}$ arestas;

(iii) entre os pares $\varepsilon$-regulares com densidade menor que $\rho$ há menos que $\rho m^{2}$ arestas;

(iv) cada $V_{i}$, para $i \in[k]$, contém no máximo $\left(\begin{array}{c}m \\ 2\end{array}\right)$ arestas.

Seja $G^{\prime \prime}=G^{\prime \prime}(\Pi, \rho, \varepsilon)$ o grafo obtido a partir de $G$ removendo as arestas descritas nos itens (i) a (iv), ou seja, aquelas arestas sobre as quais não temos controle da distribuição. Então,

$$
e(G)<\frac{1}{2}(\varepsilon n)^{2}+\varepsilon n m k+\frac{\varepsilon}{2} m^{2} k^{2}+\frac{k^{2}}{2} \rho m^{2}+\frac{1}{2} m^{2} k+e\left(G^{\prime \prime}\right),
$$

portanto, usando o limitante superior dado em (9) para $m$, temos

$$
e\left(G^{\prime \prime}\right)>e(G)-\left(\rho+4 \varepsilon+\frac{1}{k_{0}}\right) \frac{n^{2}}{2} .
$$


Esse grafo $G^{\prime \prime}$ obtido é muito útil nas aplicações, contanto que escolhamos $\rho$, $\varepsilon$ e $k_{0}$ de modo que $\left(\rho+4 \varepsilon+1 / k_{0}\right) n^{2} / 2$ seja uma fração irrelevante das arestas de $G$.

Exemplo 3. Defina $r_{k}(n)$ como a cardinalidade máxima de um subconjunto de $[n]$ que não contém uma progressão aritmética de $k$ termos, que abreviamos $k$-PA. Pelo Teorema 2 , temos que $r_{k}(n)=o(n)$. O caso $k=3$ foi provado analiticamente por Roth (1953) usando uma adaptação de uma técnica conhecida como método de Hardy-Littlewood. Vejamos uma demonstração combinatória de $r_{3}(n)=o(n)$ (cf. Graham e Rödl, 1987).

Sejam $X, Y$ e $Z$ cópias disjuntas de $[3 n]$ e $U \subseteq[n]$. Seja $S$ para o conjunto das triplas $(x, y, z) \in X \times Y \times Z$ tais que

$$
y-x=z-y=\frac{z-x}{2} \in U
$$

Seja $G=(V, E)$ o grafo com $9 n$ vértices obtido tomando-se $V=X \cup Y \cup Z$, e as arestas são os pares de $V$ contidos em alguma tripla de $S$. Então, o número de arestas em $G$ é $e(G) \geqslant 3|U| n$ e o conjunto de arestas $E$ pode ser decomposto em $e(G) / 3$ triângulos disjuntos nas arestas, que chamamos de triângulos não-espontâneos.

A primeira observação que fazemos é que, se $G$ contém um triângulo que é espontâneo, então $U$ contém uma 3-PA. De fato, se $x^{\prime}, y^{\prime}, z^{\prime}$ formam um tal triângulo, onde $y^{\prime}-x^{\prime} \neq z^{\prime}-y^{\prime}$, então para $a=y^{\prime}-x^{\prime}$ e $b=z^{\prime}-y^{\prime}$ temos $a, b \in U$ por definição, e também $\frac{a+b}{2}=\frac{z^{\prime}-x^{\prime}}{2} \in U$. Logo temos uma 3-PA em $U$.

Agora, vamos supor que $|U|=\alpha n$, para alguma constante $\alpha>0$, e vamos provar que $U$ deve conter uma progressão aritmética. Logo, é suficiente mostrar que o grafo $G$ deve conter um triângulo espontâneo.

Escreva $\nu=9 n$. Temos $e(G) \geqslant 3|U| n=3 \alpha n^{2}$, então podemos escrever $e(G)=\beta\left(\begin{array}{c}\nu \\ 2\end{array}\right)$, onde $\beta$ é uma constante fixa e independente de $n$. Aplicamos o Lema de Szemerédi em $G$ com $\varepsilon=\frac{\beta}{60}$ e $k_{0}=\left\lceil\varepsilon^{-1}\right\rceil$.

A segunda observação é que o número de arestas não contidas em pares com densidade pelo menos $\rho=\beta / 6$ é, por (10), no máximo

$$
\frac{\nu^{2}}{2}(\rho+5 \varepsilon)=\frac{\nu^{2}}{2} \frac{\beta}{4}<\frac{\beta}{3}\left(\begin{array}{l}
\nu \\
2
\end{array}\right)
$$


Removendo essas arestas obtemos $G^{\prime \prime}$ que ainda contém um triângulo $T$, pois existiam $e(G) / 3=\beta\left(\begin{array}{c}\nu \\ 2\end{array}\right) / 3$ triângulos disjuntos nas arestas (aqueles não-espontâneos) em $G$. Ainda, as três arestas do triângulo $T$ estão contidas em pares $\varepsilon$-regulares com densidade pelo menos $\beta / 6$. Vamos supor, sem perda de generalidade, que esses pares são os dados por $V_{1}, V_{2}$ e $V_{3}$.

Agora, usamos o fato que muitos vértices em pares regulares têm graus próximos.

AfIRMAÇÃo 1. Se $(U, W)$ é um par $\varepsilon$-regular com densidade $\rho_{0}=d(U, W)$, então para qualquer $Y \subseteq W$ com $|Y| \geqslant \varepsilon|W|$,

$$
\left|\left\{x \in U:|\Gamma(x) \cap Y| \leqslant\left(\rho_{0}-\varepsilon\right)|Y|\right\}\right|<\varepsilon|U| .
$$

De fato, seja $X$ o conjunto dos pontos $x \in U$ tais que $|\Gamma(x) \cap Y| \leqslant\left(\rho_{0}-\varepsilon\right)|Y|$. Então, $e(X, Y) \leqslant|X|\left(\rho_{0}-\varepsilon\right)|Y|$, portanto, $d(X, Y) \leqslant \rho_{0}-\varepsilon$ e pela $\varepsilon$-regularidade do par, concluímos que $|X|<\varepsilon|U|$.

Portanto, se $\left(V_{1}, V_{3}\right)$ e $\left(V_{2}, V_{3}\right)$ são $\varepsilon$-regulares, então existem pelo menos $(1-2 \varepsilon)\left|V_{3}\right|$ vértices em $V_{3}$, cada um ligado a pelo menos $(\beta / 6-\varepsilon)\left|V_{i}\right|$ vértices de $V_{i}$, para $i=1,2$. Fixe um deles, digamos $v$, e ponha $\Gamma_{i}(v)$ para o conjunto de vizinhos de $v$ em $V_{i}$.

De $\beta / 6-\varepsilon=9 \beta / 60>\varepsilon$ temos, pela $\varepsilon$-regularidade, que existem pelo menos $(\beta / 6-$ $\varepsilon)\left|\Gamma_{1}\right|\left|\Gamma_{2}\right|>(9 \beta / 60)^{3}\left|V_{1}\right|\left|V_{2}\right|$ arestas ligando vértices de $\Gamma_{1}(v)$ com vértices de $\Gamma_{2}(v)$. Cada aresta corresponde a um triângulo contendo $v$. Desde que, dois triângulos não-espontâneos não têm dois vértices comum, então existem no máximo $\left|V_{1}\right|=\left|V_{2}\right|$ triângulos não-espontâneos contendo o vértice $v$.

Portanto, para $n$ suficientemente grande, o grafo $G$ contém um triângulo espontâneo, ou seja, $U$ contém uma 3-PA.

Observação 4. Seguindo o argumento acima, se $V_{1}, V_{2}$ e $V_{3}$ são conjuntos disjuntos de cardinalidade $m$ que formam pares $\varepsilon$-regulares de densidade $\rho_{0}$, então nós temos o seguinte. Pela Afirmação 1 , pelo menos $(1-2 \varepsilon) m$ vértices $v_{3}$ de $V_{3}$ têm pelo menos $\left(\rho_{0}-\varepsilon\right) m$ vizinhos em $V_{1}$ e em $V_{2}$. Dado que $\rho_{0}>2 \varepsilon$ temos, pela $\varepsilon$-regularidade do par, pelo menos $\left(\rho_{0}-\varepsilon\right)^{3} m^{2}$ arestas ligando os vizinhos de $v_{3}$ em $V_{1}$ aos vizinhos desse $v_{3} \mathrm{em} V_{2}$. 
Dessa forma, o número de triângulos nessa tripla é pelo menos $t_{3}=(1-2 \varepsilon)\left(\rho_{0}-\varepsilon\right)^{3} m^{3}$. Note que $t_{3} \rightarrow \rho_{0}^{3} \mathrm{~m}^{3}$, quando $\varepsilon \rightarrow 0$. Agora, se essa tripla é formada por grafos bipartidos aleatórios com probabilidade de arestas $\rho_{0}$, então o número de triângulos tende a $\rho_{0}^{3} \mathrm{~m}^{3}$, conforme $m \rightarrow \infty$.

1.1. A idéia de uma demonstração do lema. Vejamos uma idéia da prova do Lema de Szemerédi. Seja $G$ um grafo de ordem $n$ e suponha um par $(U, W)$ de subconjuntos disjuntos de vértices que não é $\varepsilon$-regular. Tomamos os subconjuntos $U_{1}$ e $W_{1}$, de $U$ e $W$ respectivamente, que atestam a $\varepsilon$-irregularidade desse par, isto é, $\left|U_{1}\right| \geqslant \varepsilon|U|,\left|W_{1}\right| \geqslant \varepsilon|W|$ e $\left|d\left(U_{1}, W_{1}\right)-d(U, W)\right| \geqslant \varepsilon$. Sejam $U_{2}$ e $W_{2}$ os complementos de $U_{1}$ e $W_{1}$ em $U$ e $W$, respectivamente. Definimos o indice dessa partição do par $(U, W)$, pondo

$$
d=\operatorname{ind}\left(\left\{U_{1}, U_{2}\right\},\left\{W_{1}, W_{2}\right\}\right)=\frac{1}{n^{2}} \sum_{i, j}\left|U_{i}\right|\left|W_{j}\right| d\left(U_{i}, W_{j}\right)^{2} .
$$

No que segue, como ilustração, mostramos que $d \geqslant \operatorname{ind}(U, W)+\varepsilon^{4} n^{-2}|U||W|$. Essa é a idéia fundamental na prova do Lema de Szemerédi: quando há muitos pares irregulares, refinamos a partição. Como $d$ é limitado superiormente, $d<1$, e o índice cresce substancialmente no refinamento, após um número finito de refinamentos teremos uma partição $\varepsilon$-regular de $(U, W)$. Agora,

$$
d=\frac{1}{n^{2}} \sum_{i, j} \frac{e\left(U_{i}, W_{j}\right)^{2}}{\left|U_{i}\right|\left|W_{j}\right|}=\frac{1}{n^{2}}\left(\frac{e\left(U_{1}, W_{1}\right)^{2}}{\left|U_{1}\right|\left|W_{1}\right|}+\sum_{i+j>2} \frac{e\left(U_{i}, W_{j}\right)^{2}}{\left|U_{i}\right|\left|W_{j}\right|}\right) .
$$

Usando a desigualdade de Cauchy-Schwarz ${ }^{1}$

$$
\begin{aligned}
d & \geqslant \frac{1}{n^{2}}\left(\frac{e\left(U_{1}, W_{1}\right)^{2}}{\left|U_{1}\right|\left|W_{1}\right|}+\frac{\left(\sum_{i+j>2} e\left(U_{i}, W_{j}\right)\right)^{2}}{\sum_{i+j>2}\left|U_{i}\right|\left|W_{j}\right|}\right) \\
& =\frac{1}{n^{2}}\left(\frac{e\left(U_{1}, W_{1}\right)^{2}}{\left|U_{1}\right|\left|W_{1}\right|}+\frac{\left(e(U, W)-e\left(U_{1}, W_{1}\right)\right)^{2}}{|U||W|-\left|U_{1}\right|\left|W_{1}\right|}\right) .
\end{aligned}
$$

Pondo $\delta=d\left(U_{1}, W_{1}\right)-d(U, W)=e\left(U_{1}, W_{1}\right) /\left|U_{1}\right|\left|W_{1}\right|-e(U, W) /|U||W|$, temos que, $e\left(U_{1}, W_{1}\right)=\delta\left|U_{1}\right|\left|W_{1}\right|+e(U, W)\left|U_{1} \| W_{1}\right| /|U||W|$. Assim, substituindo essa igualdade na

${ }^{1} \sum \frac{\alpha_{i}^{2}}{\mu_{i}} \geqslant \frac{\left(\sum \alpha_{i}\right)^{2}}{\sum \mu_{i}}$, que segue da usual $\sum a_{i}{ }^{2} \sum b_{i}{ }^{2} \geqslant\left(\sum a_{i} b_{i}\right)^{2}$. 
equação acima e usando que $\left|U_{1}\right| \geqslant \varepsilon|U|,\left|W_{1}\right| \geqslant \varepsilon|W|$ e $|\delta| \geqslant \varepsilon$ temos (essas contas serão detalhadas adiante)

$$
n^{2} d \geqslant \frac{e(U, W)^{2}}{|U||W|}+\varepsilon^{4}|U||W|
$$

portanto $d \geqslant \operatorname{ind}(U, W)+\varepsilon^{4} n^{-2}|U||W|$.

A prova do Lema de Szemerédi (veja Seção 3.1) segue por repetidas aplicações do argumento exposto acima. Começamos com qualquer $\left(\varepsilon, k_{0}, G\right)$-eqüipartição $\Pi^{(0)}$ do conjunto de vértices $V(G)$. Se $\Pi^{(0)}$ contém mais que $\varepsilon\left(\begin{array}{c}k_{0} \\ 2\end{array}\right)$ pares irregulares nós construímos a partir de $\Pi^{(0)}$ uma $\left(\varepsilon, k_{1}, G\right)$-eqüipartição $\Pi^{(1)}$, onde $k_{1}>k_{0}$, tal que, redefinindo o índice de uma partição, ficamos com

$$
\operatorname{ind}\left(\Pi^{(1)}\right) \geqslant \operatorname{ind}\left(\Pi^{(0)}\right)+c(\varepsilon)
$$

onde $c(\varepsilon)$ é uma constante positiva que depende só de $\varepsilon$. E continuamos, dessa maneira, até que uma partição $\varepsilon$-regular seja encontrada. Como $c(\varepsilon)>0$ depende somente de $\varepsilon$ (e não depende do número de partes da partição) e o índice da partição é menor que um, deduzimos que esse procedimento termina após um número finito de passos, o qual depende de $\varepsilon$ e de $k_{0}$.

1.2. Limitações. As constantes envolvidas no Lema de Szemerédi são enormes. Por exemplo, Chvatál et al. (1983) provaram o seguinte resultado usando o lema. Dado $\Delta \in \mathbb{N}$, todo grafo $G^{n}$ de grau máximo $\leqslant \Delta$ admite número de ramsey $\mathrm{r}\left(G^{n}, G^{n}\right)$ linear, ou seja, $\mathrm{r}\left(G^{n}, G^{n}\right) \leqslant c n$, onde $c=c(\Delta)$ é uma constante positiva que depende só de $\Delta$. Por conseqüência do uso do Lema de Szemerédi, a constante $c$ é limitada superiormente por uma torre de 2's de altura $\Delta$. Recentemente, Graham et al. (2000) conseguiram uma demonstração desse resultado que evita o uso do Lema de Regularidade de Szemerédi e atinge o limitante superior $c \leqslant 2^{a \Delta(\log \Delta)^{2}}$, onde $a>0$ é uma constante.

Essas constantes tão grandes tornam proibitivo o uso do lema em problemas quantitativos. A prova original de Szemerédi (1978) fornece $K_{0}$ limitado superiormente por uma torre de 2's de altura $\varepsilon^{-5}$, portanto, as estimativas em muitas das aplicações podem ser quantitavamente ruins. Ainda, Gowers (1997) mostrou que existem grafos $G$ nos quais, para qualquer partição $(\varepsilon, k, G)$-regular dos vértices, tem-se que $k$ é maior ou igual a uma torre de 2's de altura proporcional a $\log (1 / \varepsilon)$. 
Outra limitação é a seguinte. Para um grafo $G^{n} \operatorname{com} e\left(G^{n}\right)=c n^{2-\alpha}$ arestas, onde $c, \alpha>0$ são constantes, o Lema de Szemerédi não fornece informação alguma (veja a Observação 2 na página 36 e a equação (10) na página 37 ). Ele aproxima $G^{n}$ pelo grafo vazio pois o número de arestas que desprezamos é quadrático no número de vértices. Se o grau máximo de um vértice de $G^{n}$ é $O(\sqrt{n})$ então toda $\left(\varepsilon, k, G^{n}\right)$-eqüipartição é regular porque a densidade de um subgrafo bipartido induzido sobre $\Omega(n)$ vértices é $o(1)$ e, portanto, não conseguimos medir a regularidade do par de modo eficiente.

Na próxima seção, mostramos técnicas para contornar o segundo tipo de limitação descrito acima, ou seja, vamos ver uma versão do Lema de Regularidade para tratar de grafos esparsos.

\section{Uma versão para grafos esparsos do Lema de Szemerédi}

Uma versão para grafos esparsos foi demonstrada independentemente por Rödl e Kohayakawa (veja Kohayakawa, 1997). A observação chave foi escalonar a densidade dos pares, $d_{\rho}(U, W)=(1 / \rho) d(U, W)$. Dessa forma, se existe uma constante $D$ tal que $d_{\rho}(U, W) \leqslant D$ então após um número limitado de refinamentos sucessivos de uma partição do conjunto de vértices de um grafo, chegamos numa partição com muitos pares que são regulares com respeito a densidade escalonada.

Sejam $G=(V, E)$ um grafo, constantes $D \geqslant 1,0<\eta \leqslant 1$ e $0<p \leqslant 1$ e um par de subconjuntos disjuntos $U, W \subseteq V$. Definimos a $p$-densidade entre $U, W \subseteq V$ em $G$ por

$$
d_{G, p}(U, W)=\frac{e_{G}(U, W)}{p|U||W|}
$$

e escrevemos $d_{p}(U, W)$ se $G$ é subentendido. Dizemos que $G$ é $(\eta, D, p)$-esparso, com respeito a densidade $p$, se para todos $U, W \subseteq V$ disjuntos com $|U|,|W| \geqslant \eta|V|$ temos

$$
d_{G, p}(U, W) \leqslant D
$$

Para $0<\varepsilon<1$, dizemos que $(U, W)$ é $(\varepsilon, G ; p)$-regular, ou somente $(\varepsilon ; p)$-regular quando não há perigo de confusão, se para todos $X \subseteq U$ e $Y \subseteq W$ com $|X| \geqslant \varepsilon|U|$ e $|Y| \geqslant \varepsilon|W|$ temos

$$
\left|d_{G, p}(X, Y)-d_{G, p}(U, W)\right|<\varepsilon
$$


Uma $(\varepsilon, k, G)$-eqüipartição $\Pi=\left(V_{0}, V_{1}, \ldots, V_{k}\right)$ é dita $(\varepsilon, k, G ; p)$-regular se no máximo $\varepsilon\left(\begin{array}{l}k \\ 2\end{array}\right)$ pares $\left(V_{i}, V_{j}\right)$, para $1 \leqslant i<j \leqslant k$, não são $(\varepsilon, G ; p)$-regulares.

Temos então a seguinte versão esparsa do Lema de Szemerédi.

Lema 9 (Lema de Szemerédi - versão esparsa). Dados $0<\varepsilon<1$ e $D \geqslant 1$ reais e $k_{0} \geqslant 1$ inteiro, existem constantes positivas $n_{0}=n_{0}\left(\varepsilon, D, k_{0}\right), \eta=\eta\left(\varepsilon, D, k_{0}\right)$ e $K_{0}=K_{0}\left(\varepsilon, D, k_{0}\right) \geqslant$ $k_{0}$ tais que para todo $0<p \leqslant 1$ vale o seguinte. Todo grafo $(\eta, D, p)$-esparso $G$ com pelo menos $n_{0}$ vértices admite uma partição $(\varepsilon, k, G ; p)$-regular, para algum $k_{0} \leqslant k \leqslant K_{0}$.

Observação 5. Na partição $\Pi=\left(V_{0}, V_{1}, \ldots, V_{k}\right)$ dada pelo lema acima, conseguimos garantir que $\left|V_{i}\right| \geqslant\lceil 2 \eta|V(G)|\rceil$, para todo $i \in[k]$.

Observação 6. O número de arestas num grafo $(\eta, D, p)$-esparso $G=(V, E)$ é, somando-se sobre todos os pares disjuntos $U, W \subseteq V \operatorname{com}\lceil\eta|V|\rceil$ vértices

$$
e(G)=\frac{\sum_{U, W} e(U, W)}{\frac{|U||W|}{\left(\begin{array}{c}
|W| \\
2
\end{array}\right)}\left(\begin{array}{c}
|V| \\
|U|
\end{array}\right)\left(\begin{array}{c}
|V|-|U| \\
|W|
\end{array}\right)} \leqslant \frac{\left(\begin{array}{c}
|V| \\
|U|
\end{array}\right)\left(\begin{array}{c}
|V|-|U| \\
|W|
\end{array}\right) D p|U||W|}{\frac{|U||W|}{\left(\begin{array}{c}
|V| \\
2
\end{array}\right)}\left(\begin{array}{c}
|V| \\
|U|
\end{array}\right)\left(\begin{array}{c}
|V|-|U| \\
|W|
\end{array}\right)}=D p\left(\begin{array}{c}
|V| \\
2
\end{array}\right) .
$$

Observação 7. Pelo mesmo argumento da observação anterior, podemos ver que para verificarmos que $G$ é $(\eta, D, p)$-esparso basta que tenhamos $d_{G, p}(U, W) \leqslant D$ para todos $U, W \subseteq V$ disjuntos com $|U|,|W|=\lceil\eta|V|\rceil$.

Uma versão do lema acima afirma que se colorimos as arestas de um grafo, então podemos particioná-lo em um número limitado de classes de forma que quase todos os pares são regulares em cada cor simultaneamente.

Lema 10. Dados reais $0<\varepsilon<1$ e $D \geqslant 1$ e inteiros positivos $r, k_{0} \geqslant 1$, existem constantes positivas $n_{0}=n_{0}\left(\varepsilon, D, k_{0}, r\right), \eta=\eta\left(\varepsilon, D, k_{0}, r\right)$ e $K_{0}=K_{0}\left(\varepsilon, D, k_{0}, r\right)$ tal que para todos $0<p_{1}, p_{2}, \ldots, p_{r} \leqslant 1$ vale o seguinte. Sejam $G_{1}, G_{2}, \ldots, G_{r}$ grafos $\left(\eta, D, p_{i}\right)$-esparsos, para todo $i \in[r]$, definidos sobre o mesmo conjunto de vértices $V$. Então, para algum $k_{0} \leqslant k \leqslant K_{0}$, existe uma partição de $V$ que é $\left(\varepsilon, k, G_{i} ; p_{i}\right)$-regular para todo $i \in[r]$.

Para demonstrar esse resultado, adaptamos a prova original redefinindo o índice da partição por ind $(\Pi)=\frac{1}{r} \sum_{c=1}^{r} \operatorname{ind}(\Pi)^{(c)}$, onde ind $(\Pi)^{(c)}$ é o índice da partição $\Pi$ quando levamos 
em conta somente as arestas da cor $c$, isto é, o índice para o subgrafo induzido pelas arestas da $\operatorname{cor} c \in[r]$.

\section{Uma demonstração do Lema de Szemerédi}

Nesta seção nós vamos ver uma demonstração do Lema 9. Cabe ressaltar que Kohayakawa (1997) apresenta uma demonstração para uma versão ligeiramente diferente da que enunciamos acima, embora essa versão que enunciamos (Lema 9) seja bastante usada a sua demonstração não aparece explicitamente na literatura.

São dadas as constantes $\varepsilon<1 / 2,0<\eta \leqslant 1,0<p \leqslant 1$ e $D \geqslant 1$ e $G=(V, E)$ um grafo $(\eta, D, p)$-esparso. Seja $k>0$ um inteiro e suponha que $n=|V|>k 4^{2 k+1}$. Tomamos o índice de uma partição $\Pi$ qualquer do conjunto de vértices de um grafo como

$$
\operatorname{ind}(\Pi)=\sum_{X, Y \in \Pi} \frac{|X||Y|}{n^{2}} d_{p}(X, Y)^{2},
$$

e lembramos a desigualdade de Cauchy-Schwarz:

$$
\sum \frac{\alpha_{i}^{2}}{\mu_{i}} \geqslant \frac{\left(\sum \alpha_{i}\right)^{2}}{\sum \mu_{i}}
$$

Proposição 11. Se $U, W \subseteq V$ são conjuntos disjuntos e $\Pi^{(U)}$ e $\Pi^{(W)}$ são partições de $U$ e $W$, respectivamente, então

$$
\sum_{\substack{X \in \Pi^{(U)} \\ Y \in \Pi^{(W)}}} \frac{|X||Y|}{n^{2}} d_{p}(X, Y)^{2} \geqslant \frac{|U||W|}{n^{2}} d_{p}(U, W)^{2} .
$$

Demonstração. Usando a desigualdade de Cauchy-Schwarz temos:

$$
\begin{aligned}
& \sum_{\substack{X \in \Pi^{(U)} \\
Y \in \Pi^{(W)}}} \frac{|X||Y|}{n^{2}} d_{p}(X, Y)^{2}=\frac{1}{p^{2} n^{2}} \sum_{\substack{X \in \Pi^{(U)} \\
Y \in \Pi^{(W)}}} \frac{e(X, Y)^{2}}{|X||Y|} \geqslant \frac{1}{p^{2} n^{2}} \frac{\left(\sum_{X, Y} e(X, Y)\right)^{2}}{\left(\sum_{X}|X|\right)\left(\sum_{Y}|Y|\right)}= \\
= & \frac{1}{p^{2} n^{2}} \frac{e(U, W)^{2}}{|U||W|}=\frac{|U||W|}{n^{2}} d_{p}(U, W)^{2} .
\end{aligned}
$$

Proposição 12. Se $\Pi^{(1)}$ é uma partição de $V$ que refina $\Pi^{(0)}$, então ind( $\left.\Pi^{(1)}\right) \geqslant \operatorname{ind}\left(\Pi^{(0)}\right)$. 
DEMONSTRAÇÃo. Usando a proposição anterior temos:

$$
\begin{aligned}
& \operatorname{ind}\left(\Pi^{(0)}\right)=\sum_{U, W \in \Pi^{(0)}} \frac{|U||W|}{n^{2}} d_{p}(U, W)^{2} \leqslant \sum_{U, W \in \Pi^{(0)}} \sum_{\substack{X \in \Pi^{(U)} \\
Y \in \Pi^{(W)}}} \frac{|X||Y|}{n^{2}} d_{p}(X, Y)^{2} \leqslant \\
& \sum_{U, W \in \Pi^{(0)}} \sum_{\substack{X \in \Pi^{(U)} \\
Y \in \Pi^{(W)}}} \frac{|X||Y|}{n^{2}} d_{p}(X, Y)^{2}+\sum_{U \in \Pi^{(0)}} \sum_{X, Y \in \Pi^{(U)}} \frac{|X||Y|}{n^{2}} d_{p}(X, Y)^{2}=\operatorname{ind}\left(\Pi^{(1)}\right) .
\end{aligned}
$$

Proposição 13. Se $U, W \subseteq V$ são conjuntos disjuntos e formam um par não- $(\varepsilon, G ; p)$ regular, então existem $\Pi^{(U)}=\left\{U_{1}, U \backslash U_{1}\right\}$ e $\Pi^{(W)}=\left\{W_{1}, W \backslash W_{1}\right\}$ partições de $U$ e $W$, respectivamente, tais que

$$
\sum_{\substack{X \in \Pi^{(U)} \\ Y \in \Pi^{(W)}}} \frac{|X||Y|}{n^{2}} d_{p}(X, Y)^{2} \geqslant \frac{|U||W|}{n^{2}} d_{p}(U, W)^{2}+\varepsilon^{4} \frac{|U||W|}{n^{2}}
$$

Demonstração. Ponha $U_{1}$ e $W_{1}$ subconjuntos de $U$ e $W$, respectivamente, que atestam a $(\varepsilon, p)$-irregularidade desse par, isto é, $\left|U_{1}\right| \geqslant \varepsilon|U|,\left|W_{1}\right| \geqslant \varepsilon|W|$ e $\left|d_{p}\left(U_{1}, W_{1}\right)-d_{p}(U, W)\right| \geqslant$ $\varepsilon$. Sejam $U_{2}$ e $W_{2}$ os complementos de $U_{1}$ e $W_{1}$ em $U$ e $W$, respectivamente. Definimos $\Pi^{(U)}=\left\{U_{1}, U_{2}\right\}$ e $\Pi^{(W)}=\left\{W_{1}, W_{2}\right\}$.

Agora,

$$
\begin{aligned}
\sum_{\substack{X \in \Pi^{(U)} \\
Y \in \Pi^{(W)}}} \frac{|X||Y|}{n^{2}} d_{p}(X, Y)^{2} & =\frac{1}{p^{2} n^{2}} \sum_{i, j} \frac{e\left(U_{i}, W_{j}\right)^{2}}{\left|U_{i}\right|\left|W_{j}\right|} \\
& =\frac{1}{p^{2} n^{2}}\left(\frac{e\left(U_{1}, W_{1}\right)^{2}}{\left|U_{1}\right|\left|W_{1}\right|}+\sum_{i+j>2} \frac{e\left(U_{i}, W_{j}\right)^{2}}{\left|U_{i}\right|\left|W_{j}\right|}\right) \\
& \geqslant \frac{1}{p^{2} n^{2}}\left(\frac{e\left(U_{1}, W_{1}\right)^{2}}{\left|U_{1}\right|\left|W_{1}\right|}+\frac{\left(\sum_{i+j>2} e\left(U_{i}, W_{j}\right)\right)^{2}}{\sum_{i+j>2}\left|U_{i}\right|\left|W_{j}\right|}\right) \\
& =\frac{1}{p^{2} n^{2}}\left(\frac{e\left(U_{1}, W_{1}\right)^{2}}{\left|U_{1}\right|\left|W_{1}\right|}+\frac{\left(e(U, W)-e\left(U_{1}, W_{1}\right)\right)^{2}}{|U||W|-\left|U_{1}\right|\left|W_{1}\right|}\right),
\end{aligned}
$$

onde a desigualdade acima segue de Cauchy-Schwarz. 
Pondo $\delta=d\left(U_{1}, W_{1}\right)-d(U, W)=e\left(U_{1}, W_{1}\right) /\left|U_{1}\right|\left|W_{1}\right|-e(U, W) /|U||W|$, temos que, $e\left(U_{1}, W_{1}\right)=\delta\left|U_{1}\right|\left|W_{1}\right|+e(U, W)\left|U_{1}\right|\left|W_{1}\right| /|U||W|$, portanto,

$$
\begin{aligned}
& p^{2} n^{2} \sum_{\substack{X \in \Pi^{(U)} \\
Y \in \Pi^{(W)}}} \frac{|X||Y|}{n^{2}} d_{p}(X, Y)^{2} \geqslant \\
& \frac{\left(\delta\left|U_{1}\right|\left|W_{1}\right|+\frac{e(U, W)}{|U||W|}\left|U_{1}\right|\left|W_{1}\right|\right)^{2}}{\left|U_{1}\right|\left|W_{1}\right|}+\frac{\left(e(U, W)-\frac{e(U, W)}{|U||W|}\left|U_{1}\right|\left|W_{1}\right|-\delta\left|U_{1}\right|\left|W_{1}\right|\right)^{2}}{|U||W|-\left|U_{1}\right|\left|W_{1}\right|} \\
& \geqslant \delta^{2}\left|U_{1}\right|\left|W_{1}\right|+\frac{\left|U_{1}\right|\left|W_{1}\right| e(U, W)^{2}}{|U|^{2}|W|^{2}}+2 \delta \frac{e(U, W)}{|U||W|}\left|U_{1}\right|\left|W_{1}\right|+ \\
&+ \frac{|U||W|-\left|U_{1}\right|\left|W_{1}\right|}{|U|^{2}|W|^{2}} e(U, W)^{2}+\frac{\delta^{2}\left|U_{1}\right|^{2}\left|W_{1}\right|^{2}}{|U||W|-\left|U_{1}\right|\left|W_{1}\right|}-2 \delta \frac{e(U, W)}{|U||W|}\left|U_{1}\right|\left|W_{1}\right| \\
& \geqslant \delta^{2}\left|U_{1}\right|\left|W_{1}\right|+\frac{e(U, W)^{2}}{|U||W|} \\
& \geqslant \frac{e(U, W)^{2}}{|U||W|}+\varepsilon^{4} p^{2}|U||W|,
\end{aligned}
$$

pois $\left|U_{1}\right| \geqslant \varepsilon|U|,\left|W_{1}\right| \geqslant \varepsilon|W|$ e $|\delta| \geqslant \varepsilon p$. Portanto

$$
\sum_{\substack{X \in \Pi^{(U)} \\ Y \in \Pi^{(W)}}} \frac{|X||Y|}{n^{2}} d_{p}(X, Y)^{2} \geqslant \frac{|U||W|}{n^{2}} d_{p}(U, W)^{2}+\frac{\varepsilon^{4}|U||W|}{n^{2}}
$$

como queríamos demonstrar.

Para uma $(\varepsilon, k, G)$-eqüipartição $\Pi=\left(V_{0}, V_{1}, \ldots, V_{k}\right)$ definimos o índice considerando cada elemento do conjunto excepcional $V_{0}$ como um conjunto unitário da partição, ou seja,

$$
\operatorname{ind}(\Pi)=\sum_{i=1}^{k} \sum_{j=i+1}^{k} \frac{\left|V_{i}\right|\left|V_{j}\right|}{n^{2}} d_{p}\left(V_{i}, V_{j}\right)^{2}+\sum_{i=1}^{k} \sum_{v \in V_{0}} \frac{\left|V_{i}\right|}{n^{2}} d_{p}\left(V_{i},\{v\}\right)^{2}+\operatorname{ind}\left(V_{0}\right),
$$

onde ind $\left(V_{0}\right)$ é o índice de $V_{0}$ visto como uma partição de si mesmo em conjuntos unitários.

Proposição 14. Se $\Pi^{(0)}=\left(V_{0}^{(0)}, V_{1}^{(0)}, \ldots, V_{k}^{(0)}\right)$ é uma $(\varepsilon, k, G)$-eqüipartição com mais que $\varepsilon\left(\begin{array}{l}k \\ 2\end{array}\right)$ pares de partes que não são $\varepsilon$-regulares então existe uma $\left(\varepsilon, k^{\prime}, G\right)$-eqüipartição $\Pi^{(1)}=$ $\left(V_{0}^{(1)}, V_{1}^{(1)}, \ldots, V_{k^{\prime}}^{(1)}\right)$ de $V$ definida pelos pares irregulares de $\Pi^{(0)}$ satisfazendo o seguinte.

(i) $k^{\prime}=k 4^{k}$,

(ii) $\left|V_{0}^{(1)}\right| \leqslant\left|V_{0}^{(0)}\right|+n / 4^{k}$,

(iii) todas as classes não-excepcionais de $\Pi^{(1)}$ têm a mesma cardinalidade e refinam as partes não-excepcionais de $\Pi^{(0)}$, 
(iv) $\operatorname{ind}\left(\Pi^{(1)}\right) \geqslant \operatorname{ind}\left(\Pi^{(0)}\right)+\varepsilon^{5} / 16$.

DemonstraÇÃo. Seja $\Pi^{(0)}$ uma $(\varepsilon, k, G)$-eqüipartição como enunciado. Definimos uma partição $\mathcal{P}$ de $V$ refinando $\Pi^{(0)}$ da seguinte forma. Para cada par $\left(V_{i}^{(0)}, V_{j}^{(0)}\right)$ de classes de $\Pi^{(0)}$, com $1 \leq i<j \leq k$, se o par é irregular então pomos $X_{i}(j) \subset V_{i}^{(0)}$ e $X_{j}(i) \subset V_{j}^{(0)}$ que atestam a $\varepsilon$-irregularidade do par e tomamos

$$
\Pi_{i j}=\left\{X_{i}(j), V_{i}^{(0)} \backslash X_{i}(j)\right\} \quad \text { e } \quad \Pi_{j i}=\left\{X_{j}(i), V_{j}^{(0)} \backslash X_{j}(i)\right\}
$$

as partições de $V_{i}^{(0)}$ e $V_{j}^{(0)}$ onde, pela Proposição 13,

$$
\sum_{\substack{X \in \Pi_{i j} \\ Y \in \Pi_{j i}}} \frac{|X||Y|}{n^{2}} d_{p}(X, Y)^{2} \geqslant \frac{\left|V_{i}^{(0)}\right|\left|V_{j}^{(0)}\right|}{n^{2}} d_{p}\left(V_{i}^{(0)}, V_{j}^{(0)}\right)^{2}+\varepsilon^{4} \frac{\left|V_{i}^{(0)}\right|\left|V_{j}^{(0)}\right|}{n^{2}}
$$

se o par é regular então definimos $\Pi_{i j}=\left\{V_{i}^{(0)}\right\}$ e $\Pi_{j i}=\left\{V_{j}^{(0)}\right\}$.

Assim, para cada $i \in[k]$ temos $k-1$ subconjuntos de $V_{i}^{(0)}$ onde o Diagrama de Venn nos dá uma partição de $V_{i}^{(0)}$ em $2^{k-1}$ partes, algumas delas possivelmente vazias, as quais enumeramos como $A_{i}(\lambda)$, com $\lambda \in\{0,1\}^{k-1}$, e são definidas pelos vértices $v \in V$ tais que $v \in X_{i}(j)$ se $\lambda_{j}=1$ e $v \notin X_{i}(j)$ se $\lambda_{j}=0$.

Se $\mathcal{P}=\left(V_{0}^{(0)},\left\{A_{1}(\lambda)\right\}_{\lambda}, \ldots,\left\{A_{k}(\lambda)\right\}_{\lambda}\right)$, então o índice de $\mathcal{P}$ é

$$
\begin{aligned}
& \sum_{i<j} \sum_{\lambda, \lambda^{\prime}} \frac{\left|A_{i}(\lambda)\right|\left|A_{j}\left(\lambda^{\prime}\right)\right|}{n^{2}} d_{p}\left(A_{i}(\lambda), A_{j}\left(\lambda^{\prime}\right)\right)^{2}+\sum_{i=1}^{k} \sum_{\lambda} \sum_{v \in V_{0}^{(0)}} \frac{\left|A_{i}(\lambda)\right|}{n^{2}} d_{p}\left(A_{i}(\lambda),\{v\}\right)^{2}+ \\
& +\sum_{i} \operatorname{ind}\left(\left\{A_{i}(\lambda)\right\}_{\lambda}\right)+\operatorname{ind}\left(V_{0}^{(0)}\right) .
\end{aligned}
$$

Como $\sum_{i} \operatorname{ind}\left(\left\{A_{i}(\lambda)\right\}_{\lambda}\right)>0$, e aplicando a Proposição 11, temos que o índice de $\mathcal{P}$ é pelo menos

$$
\sum_{i<j} \sum_{\substack{X \in \Pi_{i j} \\ Y \in \Pi_{j i}}} \frac{|X||Y|}{n^{2}} d_{p}(X, Y)^{2}+\sum_{i=1}^{k} \sum_{v \in V_{0}^{(0)}} \frac{\left|V_{i}^{(0)}\right|}{n^{2}} d_{p}\left(V_{i}^{(0)},\{v\}\right)^{2}+\operatorname{ind}\left(V_{0}^{(0)}\right),
$$

e pela Proposição 13, isso é pelo menos

$$
\begin{aligned}
& \sum_{i<j} \frac{\left|V_{i}^{(0)}\right|\left|V_{j}^{(0)}\right|}{n^{2}} d_{p}\left(V_{i}^{(0)}, V_{j}^{(0)}\right)^{2}+\varepsilon\left(\begin{array}{l}
k \\
2
\end{array}\right) \frac{\varepsilon^{4}\left|V_{i}^{(0)}\right|\left|V_{j}^{(0)}\right|}{n^{2}}+\sum_{i=1}^{k} \sum_{v \in V_{0}^{(0)}} \frac{\left|V_{i}^{(0)}\right|}{n^{2}} d_{p}\left(V_{i}^{(0)},\{v\}\right)^{2}+ \\
& +\operatorname{ind}\left(V_{0}^{(0)}\right) .
\end{aligned}
$$


Portanto,

$$
\operatorname{ind}(\mathcal{P}) \geqslant \operatorname{ind}\left(\Pi^{(0)}\right)+\varepsilon^{5} \frac{\left(\begin{array}{l}
k \\
2
\end{array}\right)\left|V_{i}^{(0)}\right|^{2}}{n^{2}} \geqslant \operatorname{ind}\left(\Pi^{(0)}\right)+\frac{\varepsilon^{5}}{16} .
$$

Falta construir $\Pi^{(1)}$ com todas as classes não-excepcionais de mesma cardinalidade. Ponha

$$
q=4^{k} \quad \text { e } \quad m=\left\lfloor\frac{\left|V_{i}^{(0)}\right|}{q}\right\rfloor
$$

para qualquer $i \in[k]$. Observe que $\left|V_{i}^{(0)}\right| \geqslant(1-\varepsilon)|V| / k>|V| /(2 k) \geqslant 2 \cdot 4^{2 k}$, logo $q^{2}=4^{2 k} \leqslant$ $\left|V_{i}^{(0)}\right|=m q+r<(m+1) q$, onde $0 \leqslant r<q \leqslant m$, portanto

$$
q=\left\lfloor\frac{\left|V_{i}^{(0)}\right|}{m}\right\rfloor \text {. }
$$

Agora, em cada $A_{i}(\lambda)$ escolhemos $q_{\lambda}=\left\lfloor\left|A_{i}(\lambda)\right| / m\right\rfloor$ subconjuntos dois-a-dois disjuntos, cada um com exatamente $m$ vértices, e cuja união denotamos por $\bar{A}_{i}(\lambda)$. Logo $\left|A_{i}(\lambda) \backslash \bar{A}_{i}(\lambda)\right| \leqslant$ $m$ e sobram

$$
\left|V_{i}^{(0)} \backslash \bigcup_{\lambda} \bar{A}_{i}(\lambda)\right|=m q+r-\sum_{\lambda} q_{\lambda} m=m\left(q-\sum_{\lambda} q_{\lambda}\right)+r
$$

vértices. Portanto, temos um adicional de $q-\sum_{\lambda} q_{\lambda}$ subconjuntos dois-a-dois disjuntos de $V_{i}^{(0)}$ com $m$ vértices cada um. Dessa forma, particionamos $V_{i}^{(0)}$ em $q=4^{k}$ subconjuntos $V_{i}(1), \ldots, V_{i}(q)$. Esses subconjuntos formam as partes não-excepcionais de $\Pi^{(1)}$. Os no máximo $m-1$ vértices que podem ter sobrado são colocados no conjunto excepcional,

$$
V_{0}^{(1)}=V \backslash \bigcup_{i=1}^{k} \bigcup_{s=1}^{q} V_{i}(s)
$$

portanto,

$$
\left|V_{0}^{(1)}\right| \leqslant\left|V_{0}^{(0)}\right|+k m \leqslant\left|V_{0}^{(0)}\right|+\sum_{i=1}^{k} \frac{\left|V_{i}^{(0)}\right|}{q} \leqslant\left|V_{0}^{(0)}\right|+\frac{\left|V \backslash V_{0}^{(0)}\right|}{q} \leqslant\left|V_{0}^{(0)}\right|+\frac{|V|}{4^{k}} .
$$

Dessa maneira, $V_{0}^{(1)}$ e $\left(\left(V_{i}(s)\right)_{s=1}^{q}\right)_{i=1}^{k}$ definem a partição $\Pi^{(1)}$ de $V$. Como $\Pi^{(1)}$ refina $\mathcal{P}$ temos

$$
\operatorname{ind}\left(\Pi^{(1)}\right) \geqslant \operatorname{ind}(\mathcal{P}) \geqslant \operatorname{ind}\left(\Pi^{(0)}\right)+\frac{\varepsilon^{5}}{16}
$$


3.1. A demonstração do Lema de Szemerédi. Dados $\varepsilon<1 / 2, D \geqslant 1$ e $k_{0} \geqslant 1$ ponha $T=\left\lfloor 16 D^{2} \varepsilon^{-5}\right\rfloor$, e $t_{0}$ o menor inteiro tal que $4^{t_{0}} \geqslant 2^{T+2} \varepsilon^{-1}$. Defina $\pi(0)=t_{0}$ e, indutivamente, $\pi(t)=\pi(t-1) 4^{\pi(t-1)}$.

Tome

$$
\eta=\min \left\{\eta_{0}(\pi(t)): 0 \leqslant t \leqslant T\right\} \text { e } K_{0}=\max \left\{2 \varepsilon^{-1} t_{0}, \max \left\{n_{0}(\pi(t)): 0 \leqslant t \leqslant T\right\}\right\},
$$

onde $\eta_{0}(k)=\left(k 4^{k+1}\right)^{-1}$ e $n_{0}(k)=k 4^{2 k+1}$.

Fixamos $0<p<1$ e seja $G=G^{n}$ um grafo $(\eta, D, p)$-esparso com $n \geqslant K_{0}$ vértices. Também, seja $\mathcal{T}$ o conjunto dos inteiros $t \geqslant 0$ tais que existe uma $(\varepsilon, \pi(t), G)$-eqüipartição $\Pi^{(t)}$ tal que

(i) $\operatorname{ind}\left(\Pi^{(t)}\right) \geqslant t \varepsilon^{5} / 16$, e

(ii) $\left|V_{0}^{(t)}\right| \leqslant \varepsilon n\left(1-2^{-t-1}\right)$.

Tal partição existe para $t=0$ : tome $m=\left\lfloor n / t_{0}\right\rfloor$ e $\Pi^{(0)}$ a partição de $V(G)$ em partes de cardinalidade $m$, exceto possivelmente uma que terá cardinalidade $<t_{0} \leqslant \varepsilon K_{0} / 2 \leqslant \varepsilon n / 2=$ $\varepsilon n(1-1 / 2)$. Como ind $\left(\Pi^{(0)}\right) \geqslant 0$ os itens (i) e (ii) valem, ou seja $0 \in \mathcal{T}$.

Da escolha de $\eta$ temos que $\left|V_{i}^{(t)}\right|>\eta n$. De fato,

$$
\left|V_{i}^{(t)}\right| \geqslant(1-\varepsilon) \frac{n}{\pi(t)}=(1-\varepsilon) \frac{n}{\pi(t-1) 4^{\pi(t-1)}}=(1-\varepsilon) 4 n \eta_{0}(\pi(t-1))
$$

e, como $\varepsilon<1 / 2$, logo $\left|V_{i}^{(t)}\right|>2 n \eta_{0}(\pi(t-1)) \geqslant 2 \eta n$, logo a $p$-densidade entre os pares de conjuntos da partição é limitada superiormente por $D$, portanto, ind( $\left.\Pi^{(t)}\right)<D^{2}$ e nós temos que $\mathcal{T}$ é limitado superiormente. Considere $t$ o maior inteiro em $\mathcal{T}$ e escreva $\Pi^{(t)}=$ $\left(V_{0}^{(t)}, V_{1}^{(t)}, \ldots, V_{\pi(t)}^{(t)}\right)$.

Se uma fração maior que $\varepsilon$ dos pares de partes de $\Pi^{(t)}$ são $\varepsilon$-irregulares então existe um refinamento $\Pi^{(t+1)}$ de $\Pi^{(t)}$ com

$$
\operatorname{ind}\left(\Pi^{(t+1)}\right) \geqslant \operatorname{ind}\left(\Pi^{(t)}\right)+\frac{\varepsilon^{5}}{16} \geqslant(t+1) \frac{\varepsilon^{5}}{16}
$$

e, usando que $4^{\pi\left(t_{0}\right)} \geqslant 4^{\pi(0)}=4^{t_{0}} \geqslant \varepsilon^{-1} 2^{t+2}$, temos

$$
\left|V_{0}^{(t+1)}\right| \leqslant\left|V_{0}^{(t)}\right|+n 4^{-\pi(t)} \leqslant \varepsilon n\left(1-\frac{1}{2^{t+1}}+\frac{1}{\varepsilon 4^{\pi(t)}}\right) \leqslant \varepsilon n\left(1-\frac{1}{2^{t+2}}\right),
$$

portanto $t+1 \in \mathcal{T}$, contrariando a maximalidade de $t$. 


\section{Considerações finais}

Na demonstração da versão para grafos esparsos do Lema de Szemerédi o fato do grafo ser $(\eta, D, p)$-esparso, sendo, portanto, a $p$-densidade de um par limitada, isto é, $d_{p}(U, W) \leqslant D$, foi usado para garantir que o índice de uma partição é limitado superiormente, ou seja, ind(ח) $<D^{2}$. Então poderíamos chamar de esparso um grafo no qual toda partição fosse limitada.

Essa alternativa foi tomada recentemente por Euczak (2000) que provou a seguinte variante do Lema de Szemerédi para grafos esparsos. Seja $G$ um grafo, $f:[0, \infty) \rightarrow[0, \infty)$ uma função e $\Pi=\left(V_{1}, \ldots, V_{k}\right)$ uma partição balanceada de $V(G)$. Defina o índice da partição $\Pi$ por

$$
\operatorname{ind}_{f}(\Pi)=\sum_{1 \leqslant i<j \leqslant k} \frac{\left|V_{i}\right|\left|V_{j}\right|}{|V|^{2}} f\left(d_{p}\left(V_{i}, V_{j}\right)\right),
$$

e chame o grafo $G$ de $(f, L, b)$-esparso, para reais $L$ e $b>1$, se para toda $k$-partição balanceada $\Pi$ de $V$ com $2 \leqslant k \leqslant L$, temos $\operatorname{ind}_{f}(\Pi) \leqslant b$. Aqui, a $p$-densidade de um par de subconjuntos disjuntos é tomada para o fator de escala $p=e(G) /|V(G)|^{2}$.

Lema 15. Dados uma função positiva e estritamente convexa $f$ e reais $\varepsilon>0$ e $b>1$, existe um $L$ tal que todo grafo $G$ de ordem pelo menos $L$ e $(f, L, b)$-esparso admite uma partição balanceada $(\varepsilon, k)$-regular para algum $1 / \varepsilon \leqslant k \leqslant L$.

Para o leitor interessado em conhecer mais sobre o Lema de Regularidade sugerimos Komlós e Simonovits (1996), onde encontramos vários exemplos de aplicações além de versões algorítmicas e para hipergrafos do Lema de Regularidade. Aliás, um problema que ainda resta com relação ao Lema de Regularidade é o de descobrir uma versão tão poderosa quanto a versão original para grafos esparsos e para hipergrafos. 


\section{CAPíTULO 1}

\section{CONTAGEM DE $C^{\ell}$ 's EM GRAFOS ESPARSOS}

Lembramos que na demonstração de $r_{3}(n)=o(n)$, Exemplo 3 na página 38 (veja também a observação que segue o exemplo), a existência de uma tripla de subconjuntos de vértices $V_{1}, V_{2}, V_{3} \subseteq V(G)$ dois-a-dois $\varepsilon$-regulares implicou na existência de muitos triângulos em $G$. Entretanto, quando o grafo $G$ é esparso esse "esquema de imersão", que está descrito precisamente no Lema 16 abaixo, não funciona.

Neste capítulo veremos um resultado que, como caso particular, mostra que o número de grafos tripartidos esparsos, com as partes $\varepsilon$-regulares, com $M$ arestas e que não contêm triângulos é superexponencialmente pequeno em $M$.

Kohayakawa e Kreuter (1997) mostraram que numa dada família de grafos $\mathcal{F}$, que será descrita em breve, a fração de grafos sem circuitos de um comprimento fixo é superexponencialmente pequena no número de arestas $M$. Neste capítulo, provaremos o Teorema 20, o qual chamamos de Lema de Contagem, que é um refinamento desse resultado e diz que a fração de grafos de $\mathcal{F}$ com $o(M)$ circuitos de um comprimento fixo é superexponencialmente pequena no número de arestas $M$.

\section{Introdução}

Quando temos um grafo $G$ denso, o Lema de Regularidade nos garante que se $|V(G)|$ é suficientemente grande, então conseguimos uma cópia de um grafo "pequeno" $H$ em $G$.

Vamos fazer o parágrafo anterior mais preciso. Dados um grafo $R$, reais $\rho>\varepsilon>0$ e um inteiro positivo $m$, construímos o grafo $G=G(R ; m, \rho, \varepsilon)$ da seguinte forma. Para cada vértice de $R$ tomamos um conjunto independente com $m$ pontos. Agora, substituímos cada aresta de $R$ por arestas ligando os respectivos conjuntos independentes de modo que esse par de conjuntos independentes formem um par $\varepsilon$-regular de densidade pelo menos $\rho$. Dado um inteiro $t>0$, denotamos por $R(t)$ o grafo obtido pelo procedimento acima quando os pares 
de conjuntos independentes cujos respectivos vértices são arestas em $R$ induzem subgrafos bipartidos completos.

Agora, para todo grafo $H$ com $h$ vértices e grau máximo $\Delta$, se $H \subseteq R(t)$, então $H \subseteq$ $G(R ; m, \rho, \varepsilon)$ desde que $\varepsilon$ seja suficientemente pequeno e $m$ seja suficientemente grande. Mais precisamente, se $\varepsilon_{0}=(\rho-\varepsilon)^{\Delta} /(2+\Delta)$, então vale o seguinte resultado de imersão.

Lema 16 (Komlós e Simonovits, 1996). Se $\varepsilon \leqslant \varepsilon_{0}$ e $m \geqslant(t-1) \varepsilon_{0}{ }^{-1}$, então $H \subseteq G e$, mais que isso, o número de cópias rotuladas de $H$ em $G$ é maior que

$$
\left(\left((\rho-\varepsilon)^{\Delta}-\Delta \varepsilon\right) m-(t-1)\right)^{h} \geqslant\left(\varepsilon_{0} m\right)^{h} .
$$

Ingenuamente, estamos na seguinte situação. Seja $G=G^{n}$ um grafo denso, digamos que $\operatorname{com} \beta n^{2}$ arestas. Aplicamos o Lema de Regularidade para obtermos uma $(\varepsilon, k)$-eqüipartição $\Pi$ de $V(G)$. Seja $R$ o grafo definido pondo um vértice para cada classe de $\Pi$ e uma aresta ligando cada dois vértices que representam pares $(\varepsilon, G)$-regulares com densidade pelo menos $\rho$. Pela equação (10), página 37 , pelo menos $\beta n^{2}-\left(\rho+4 \varepsilon+1 / k_{0}\right) n^{2} / 2$ arestas de $G$ ligam vértices em pares regulares densos. Há no máximo $(n / k)^{2}$ arestas em cada par, logo existem pelo menos $\left(2 \beta-\rho-4 \varepsilon-1 / k_{0}\right) k^{2} / 2$ pares densos em $\Pi$, ou seja, existe $\delta=\delta\left(\beta, \rho, \varepsilon, k_{0}\right)>0$ tal que $e(R)>(1-\delta)\left(\begin{array}{c}k \\ 2\end{array}\right)$. Se escolhemos $\rho, \varepsilon$ e $k_{0}$ de modo que $\delta$ é suficientemente pequeno, temos que $H \subseteq R$ (veja na página 28 a definição do número de Turán) e pelo Lema 16, temos $H \subseteq G$.

Para grafos esparsos, digamos com $\Theta\left(p\left(\begin{array}{l}n \\ 2\end{array}\right)\right)$ arestas, onde $0<p=p(n)<1$, não conhecemos um equivalente ao Lema 16 e uma generalização óbvia não funciona, como mostra o seguinte resultado.

Lema 17 (Kohayakawa e Rödl, 2001). Para todo $0<\varepsilon<1$ existe $0<p<1$ e um inteiro $m_{0} \geqslant 1$ tais que o seguinte vale. Para todo $m \geqslant m_{0}$ existe um grafo tripartido $G$ sobre as classes de vértices $V_{1}, V_{2}$ e $V_{3}$, todas de cardinalidade $m$, tal que para todo $1 \leqslant i<j \leqslant 3$ temos

(i) $e\left(V_{i}, V_{j}\right)=M=\left\lfloor p m^{2}\right\rfloor$,

(ii) $\left(V_{i}, V_{j}\right)$ é $(\varepsilon ; p)$-regular, mas 
(iii) $G$ não contém triângulo como subgrafo.

Na próxima seção, enunciamos um resultado que contorna essa situação no caso de circuitos de tamanho fixo.

O modo como usamos o Lema de Contagem encerra uma técnica desenvolvida por Füredi (1994) que foi resumida por Kohayakawa e Rödl (2001) da seguinte forma. Seja $P$ uma propriedade de grafos e suponha que queremos mostrar que todo subgrafo suficientemente grande $H \subseteq G_{n, p}$ satisfaz $P$ quase certamente, onde suficientemente grande significa

$$
m=v(H) \geqslant m_{0}=m_{0}(n) \quad \text { e } \quad M=e(H) \geqslant \beta p\left(\begin{array}{c}
m \\
2
\end{array}\right)
$$

para uma constante $\beta$ que não depende de $n$. Assim, estamos falando de uma quantidade de subgrafos de $G_{n, p}$ que é exponencial em $\Omega\left(p m^{2}\right)$. A técnica de Füredi consiste em mostrar que a família de contra-exemplos para $P$ é assintoticamente pequena, de fato superexponencialmente pequena, e então mostrar que esses raros contra-exemplos quase certamente não aparecem em $G_{n, p}$.

Para sermos mais precisos, consideramos uma família $\mathcal{B}$ de grafos e escrevemos $\mathcal{B}(m, M)$ para os grafos de $\mathcal{B}$ sobre $[m]$ com $M$ arestas. Dada uma constante positiva $\alpha$, dizemos que $\mathcal{B}$ é uma família $\alpha$-magra se existe $m_{1}$ tal que, para todo $0 \leqslant M \leqslant\left(\begin{array}{c}m \\ 2\end{array}\right)$,

$$
\text { se } m \geqslant m_{1} \text {, então }|\mathcal{B}(m, M)| \leqslant \alpha^{M}\left(\begin{array}{c}
m \\
2 \\
M
\end{array}\right) \text {. }
$$

Lema 18 (Kohayakawa e Rödl, 2001). Sejam $0<p=p(n)<1$ e $m_{0}=m_{0}(n)$ funções tais que $p m_{0} \gg \ln n$. Então, para qualquer $\beta>0$ constante, existe $\alpha>0$ tal que se $\mathcal{B}$ é uma familia $\alpha$-magra vale o seguinte: quase certamente o grafo aleatório $G_{n, p}$ não contém cópias de qualquer membro de $\mathcal{B}$ que satisfaz (13).

A demonstração é obtida estimando-se o número esperado de cópias de elementos de $\mathcal{B}$ em $G_{n, p}$, que é $o(1)$, e concluída pela desigualdade de Markov.

$\mathrm{Na}$ próxima seção vamos mostrar que uma determinada família de grafos é magra com respeito a propriedade "conter circuito de comprimento $\ell$ ". 


\section{Lema de Contagem}

Suponhamos que sejam dados inteiros $m>0$ e $\ell \geqslant 3$, e um vetor de conjuntos dois-a-dois disjuntos $\mathrm{V}^{(m)}=\left(V_{i}\right)_{i=1}^{\ell}$, cada um de cardinalidade $m$. No que segue, os índices dos $V_{i}$ 's serão considerados módulo $\ell$. Sejam $B>0, C \geqslant 1, D \geqslant 1, \varepsilon \leqslant 1, \rho_{0} \leqslant 1$ reais positivos e $M \geqslant 1$ inteiro.

Chamamos um grafo $F$ de

$$
\left(\varepsilon, \rho_{0}, B, C, D ; \mathbf{V}^{(m)}, M\right)-\text { grafo }
$$

se

(i) $E(F)=\bigcup_{i=1}^{\ell} E\left(V_{i}, V_{i+1}\right)$ e $|E(F)|=M$.

(ii) Para todo $1 \leqslant i \leqslant \ell$ os pares $\left(V_{i}, V_{i+1}\right)$ são $(\varepsilon, F ; \bar{p})$-regulares, com $\bar{p}$-densidade

$$
\rho_{0} \leqslant d_{F, \bar{p}}\left(V_{i}, V_{i+1}\right) \leqslant D
$$

onde $\bar{p}=B m^{-1+1 /(\ell-1)}$.

(iii) Para todo $U \subseteq V_{i}$ e todo $W \subseteq V_{i+1}$, onde $1 \leqslant i<\ell-1$, tais que

$$
|U| \leqslant|W| \leqslant \bar{p} m|U| \leqslant(\bar{p} m)^{\ell-2},
$$

temos

$$
e(U, W) \leqslant C|W| \text { : }
$$

Kohayakawa e Kreuter (1997) mostram que a família dos $\left(\varepsilon, \rho_{0}, B, C, D ; \mathrm{V}^{(m)}, M\right)$-grafos que não contêm circuitos de comprimento $\ell$ é $\alpha$-magra:

LEMA 19. Dado um inteiro $\ell \geqslant 3$ e dados reais $0<\alpha \leqslant 1,0<\rho_{0} \leqslant 1, C \geqslant 1 e$ $D \geqslant 1$ existem constantes positivas $\bar{\varepsilon}=\bar{\varepsilon}\left(\ell, \alpha, \rho_{0}, C, D\right) \leqslant 1, \bar{B}_{0}=\bar{B}_{0}\left(\ell, \alpha, \rho_{0}, C, D\right)>0$, e $\bar{m}_{0}=\bar{m}_{0}\left(\ell, \alpha, \rho_{0}, C, D\right)$ tais que para todos inteiros $m \geqslant \bar{m}_{0}$ e $M \geqslant 1$, e todo real $B \geqslant \bar{B}_{0}$, o seguinte vale. O número de $\left(\bar{\varepsilon}, \overline{\rho_{0}}, B, C, D ; \mathrm{V}^{(m)}, M\right)$-grafos sem circuitos de comprimento $\ell$ é no máximo

$$
\alpha^{M}\left(\begin{array}{c}
(\ell+2) m^{2} \\
M
\end{array}\right) \text {. }
$$


Neste capítulo mostraremos o seguinte resultado.

Teorema 20 (Lema de Contagem). Dado um inteiro $\ell \geqslant 3$ e dados reais $\sigma>0,0<$ $\alpha \leqslant 1,0<\rho_{0} \leqslant 1, C \geqslant 1$ e $D \geqslant 1$ existem constantes positivas $\varepsilon=\varepsilon\left(\ell, \sigma, \alpha, \rho_{0}, C, D\right) \leqslant 1$, $B_{0}=B_{0}\left(\ell, \sigma, \alpha, \rho_{0}, C, D\right)>0$, e $m_{0}=m_{0}\left(\ell, \sigma, \alpha, \rho_{0}, C, D\right)$ tais que para todos inteiros $m \geqslant m_{0}$ e $M \geqslant 1$, e todo real $B \geqslant B_{0}$, o seguinte vale. O número de $\left(\varepsilon, \rho_{0}, B, C, D ; \mathrm{V}^{(m)}, M\right)$-grafos contendo menos que $\sigma m^{\ell /(\ell-1)}$ circuitos de comprimento $\ell$ é no máximo

$$
\alpha^{M}\left(\begin{array}{c}
(\ell+2) m^{2} \\
M
\end{array}\right)
$$

Demonstração. Para demonstrar esse resultado, vamos nos basear no seguinte fato. Se $(U, W)$ é $(\varepsilon ; p)$-regular com $T$ arestas, densidade pelo menos $\rho_{0}$ e no máximo $D$, então removendo $\delta T$ arestas de $E(U, W)$ ficamos com um par $\left(\varepsilon^{\prime} ; p\right)$-regular com $(1-\delta) T$ arestas e densidade pelo menos $(1-\delta) \rho_{0}$ e no máximo $D$, para $\varepsilon^{\prime}=\varepsilon+\delta D$.

Dados $\ell \geqslant 3, \sigma>0,0<\alpha \leqslant 1,0<\rho_{0} \leqslant 1, C \geqslant 1$ e $D \geqslant 1$ como no enunciado do Lema de Contagem, definimos

$$
\begin{aligned}
\bar{\alpha} & =\left(\frac{\alpha}{2 \mathrm{e}}\right)^{1-\sigma}, \\
\overline{\rho_{0}} & =(1-\sigma) \rho_{0},
\end{aligned}
$$

e tomamos as constantes dadas pelo Lema 19

$$
\begin{aligned}
\bar{\varepsilon} & =\bar{\varepsilon}\left(\ell, \bar{\alpha}, \overline{\rho_{0}}, C, D\right) \leqslant 1, \\
\overline{B_{0}} & =\bar{B}_{0}\left(\ell, \bar{\alpha}, \overline{\rho_{0}}, C, D\right)>0, \\
\bar{m}_{0} & =\bar{m}_{0}\left(\ell, \bar{\alpha}, \overline{\rho_{0}}, C, D\right) .
\end{aligned}
$$

Observamos que $M \geqslant \rho_{0} \bar{p} m^{2} \ell=\rho_{0} B \ell m^{\ell /(\ell-1)}$. Seja $\delta=\delta(\sigma)$ uma constante tal que $\sigma m^{\ell /(\ell-1)} \leqslant \delta M$ e defina $\varepsilon=\bar{\varepsilon}-\delta D, B_{0}=\bar{B}_{0}$ e $m_{0}=\bar{m}_{0}$.

Todo $\left(\varepsilon, \rho_{0}, B, C, D ; \mathrm{V}^{(m)}, M\right)$-grafo $G$ contendo menos que $\delta M$ circuitos de comprimento $\ell$ pode ser visto como um par $(H, J)$, onde $H$ é um $\left(\bar{\varepsilon}, \overline{\rho_{0}}, B, C, D ; \mathrm{V}^{(m)}, M^{\prime}\right)$-grafo sem circuitos $C^{\ell}$, para $M^{\prime}=(1-\delta) M$, e $J$ é um subgrafo de $G$ com no máximo $\delta M$ arestas cuja remoção destrói todos os circuitos de comprimento $\ell$ de $G$. 
Assim, pelo Lema 19, o número de tais grafos $G$ é menor que

$$
\bar{\alpha}^{(1-\delta) M}\left(\begin{array}{c}
(\ell+2) m^{2} \\
(1-\delta) M
\end{array}\right)\left(\begin{array}{c}
(\ell+2) m^{2} \\
\delta M
\end{array}\right) \leqslant\left(\frac{\alpha}{2 \mathrm{e}}\right)^{M}\left(\begin{array}{c}
2(\ell+2) m^{2} \\
M
\end{array}\right) \leqslant \alpha^{M}\left(\begin{array}{c}
(\ell+2) m^{2} \\
M
\end{array}\right),
$$

onde as desigualdades seguem da identidade de Vandermonde

$$
\sum_{x+y=z}\left(\begin{array}{l}
a \\
x
\end{array}\right)\left(\begin{array}{l}
b \\
y
\end{array}\right)=\left(\begin{array}{c}
a+b \\
z
\end{array}\right),
$$

e de $\left(\begin{array}{l}a \\ x\end{array}\right) \leqslant(\mathrm{e} a / x)^{x} \leqslant \mathrm{e}^{x}\left(\begin{array}{l}a \\ x\end{array}\right)$

\section{Considerações finais}

Kohayakawa et al. (1997) conjecturaram que vale o seguinte resultado.

Conjectura 21 (Kohayakawa et al., 1997). Para todo grafo não-vazio $H$ de ordem pelo menos 3 e para todo $0<p=p(n) \leqslant 1$ tal que $p n^{1 / m_{2}(H)} \rightarrow \infty$ quando $n \rightarrow \infty$, onde

$$
m_{2}(H)=\max \left\{\frac{e(J)-1}{v(J)-2}: J \subseteq H, v(J)>2\right\}
$$

temos que

$$
\operatorname{ex}\left(G_{n, p}, H\right)=\left(1-\frac{1}{\chi(H)-1}+o(1)\right) e\left(G_{n, p}\right)
$$

quase certamente.

Além disso, quase certamente vale a seguinte afirmação. Se $\chi(H) \geqslant 3$, então para todo real $\varepsilon>0$ existe $\delta=\delta(\varepsilon)$ tal que todo subgrafo $J \subseteq G_{n, p}$ que não contém $H$ e com e $(J) \geqslant$ $(1-\delta) \operatorname{ex}\left(G_{n, p}, H\right)$ pode ser feito $(\chi(H)-1)$-partido removendo-se no máximo ee $(J)$ arestas de $J$.

Para a primeira parte da conjectura acima, os casos $H=K^{3}$ e $H=C^{4}$ foram provados por Frankl e Rödl (1986) e Füredi (1994), respectivamente. Kohayakawa et al. (1997) provaram o caso $H=K^{4}$ e observaram que uma aplicação simples do Lema 9 prova a conjectura quando $H$ é uma floresta. O caso $H=C^{\ell}$ foi provado por Haxell et al. $(1995 \mathrm{~b}, 1996)$.

Vamos descrever uma conjectura de Kohayakawa et al. (1997) sobre contagem de grafos a qual, se verdadeira, implica a equação (18). Sejam $m$ um inteiro positivo e $H$ um grafo com $V(H)=\left\{v_{1}, \ldots, v_{h}\right\}$, para $h \geqslant 3,0<p=p(m) \leqslant 1$ e seja $\mathrm{V}^{(m)}=\left(V_{i}\right)_{i=1}^{h}$ uma família 
de conjuntos dois-a-dois disjuntos, cada um com cardinalidade $m$. Dados reais $0<\varepsilon \leqslant 1$, $D \geqslant 1$ e $0<\rho_{0} \leqslant 1$ e um inteiro positivo $M$, dizemos que um grafo $h$-partido $F$ com partição $V(F)=V_{1} \cup \cdots \cup V_{h}$ e com $M$ arestas é um

$$
\left(\varepsilon, \rho_{0}, H ; \mathrm{V}^{(m)}, M\right) \text {-grafo }
$$

se $v_{i} v_{j} \in E(H)$, então $\left(V_{i}, V_{j}\right)$ é $(\varepsilon, F, p)$-regular com $\rho_{0} \leqslant d_{F, p}\left(V_{i}, V_{j}\right) \leqslant D$, para todo $1 \leqslant i<$ $j \leqslant h$.

Conjectura 22 (Kohayakawa et al., 1997). Dados $0<\alpha \leqslant 1$ e $0<\rho_{0} \leqslant 1$ existem constantes $\varepsilon=\varepsilon\left(\alpha, \rho_{0}\right)>0$ e $C=C\left(\alpha, \rho_{0}\right)$ tais que se $p=p(m) \geqslant C m^{-1 / m_{2}(H)}$, então o número de $\left(\varepsilon, \rho_{0}, H ; \mathrm{V}^{(m)}, M\right)$-grafos livres de $H$ é no máximo

$$
\alpha^{M}\left(\begin{array}{c}
\left(\begin{array}{c}
h \\
2
\end{array}\right) m^{2} \\
M
\end{array}\right)
$$

para todo $m$ suficientemente grande.

A dedução da primeira parte da Conjectura 21 a partir da Conjectura 22 pode ser feita adaptando-se alguns cálculos do próximo capítulo (veja Proposição 28). 


\section{CAPíTULO 2}

\section{A DENSIDADE DE $\vec{C}^{\ell}$ EM GRAFOS ORIENTADOS ESPARSOS E UMA CONJECTURA DE WOODALL}

Chamamos o par $\vec{G}=(V, E)$ um grafo orientado se o conjunto de vértices $V$ é finito e o conjunto de arestas orientadas $E \subseteq V \times V$, que nós chamamos de arcos, é tal que $(v, v) \notin E$ qualquer que seja $v \in V$ e se $(u, v) \in E$, então $(v, u) \notin E$.

Ainda, temos o seguinte modelo probabilístico para um grafo orientado. Lembramos que, dado $0<p \leqslant 1$, escrevemos $G_{n, p}$ para um grafo aleatório no modelo binomial, onde $n$ é o número de vértices e $p$ é a probabilidade de arestas. A partir de $G_{n, p}$ nós obtemos um grafo orientado aleatório $\vec{G}_{n, p}$ pondo, para cada aresta $\{u, v\} \in E\left(G_{n, p}\right)$ em $G_{n, p}$,

$$
\mathbb{P}\left\{(u, v) \in E\left(\vec{G}_{n, p}\right)\right\}=1-\mathbb{P}\left\{(v, u) \in E\left(\vec{G}_{n, p}\right)\right\}=\frac{1}{2},
$$

com todas as orientações independentes.

Neste capítulo, mostraremos que existe um grafo orientado $\vec{G}$ sobre $n$ vértices tal que qualquer subconjunto de $E(\vec{G})$ com pelo menos $(1 / 2+\beta) e(\vec{G})$ arcos induz um circuito orientado de comprimento $\ell$, para todo $\ell \geqslant 3$, para qualquer $\beta>0$ e para todo $n$ suficientemente grande. Também, veremos como esse resultado gera uma família infinita de contra-exemplos para uma generalização de uma conjectura de Woodall.

No que segue sempre assumimos que $n$ é grande o suficiente para todas as desigualdades valerem.

\section{Introdução}

O teorema principal desse capítulo, Teorema 24, está relacionado com uma conjectura de Woodall a qual passamos a descrever agora. Dado um grafo orientado $\vec{G}=(V, E)$, dizemos que um subconjunto de $\operatorname{arcos} B \subseteq E$ é um corte orientado em $\vec{G}$ quando existe um subconjunto de vértices $W \subseteq V$ tal que $B=E(\vec{G}) \cap(W \times \bar{W})$ e $E(\vec{G}) \cap(\bar{W} \times W)=\emptyset$, onde $\bar{W}=V \backslash W$. 
Um subconjunto de $\operatorname{arcos} F \subseteq E$ é uma transversal de cortes se for uma transversal da família de cortes orientados de $G$, ou seja, se $F \cap B \neq \emptyset$ para todo corte orientado $B$ em $\vec{G}$.

Woodall (1978) conjecturou que para qualquer grafo orientado $\vec{G}$, a cardinalidade de um corte orientado de tamanho mínimo em $\vec{G}$ é igual a cardinalidade máxima de uma família de transversais duas-a-duas disjuntas de cortes orientados. Para alguns casos particulares, a resposta afirmativa para tal conjectura é conhecida. Feofiloff e Younger (1987), e independentemente Schrijver (1982), provaram a conjectura para grafos orientados fonte-sorvedouro conexos. Um grafo orientado é chamado fonte-sorvedouro conexo se é acíclico e cada fonte é ligada a todo sorvedouro por um caminho orientado. Lee e Wakabayashi (veja Lee, 1999) recentemente verificaram a conjectura para grafos orientados série-paralelos. Um grafo orientado é chamado série-paralelo se o grafo subjacente não contém uma subdivisão do $K^{4}$.

Vejamos como o Teorema 24 abaixo está relacionado à conjectura de Woodall. Chamamos de cintura orientada do grafo orientado $\vec{G}$ o mínimo dos comprimentos dos circuitos orientados de $\vec{G}$. Chamamos um subconjunto de $\operatorname{arcos} D \subseteq E$ de transversal de circuitos se for uma transversal da família dos circuitos orientados de $\vec{G}$, ou seja, para todo circuito orientado $\vec{C}$, temos $\vec{C} \cap \vec{G} \neq \emptyset$. Daqui em diante, chamaremos resumidamente de transversal qualquer transversal da família dos circuitos orientados.

O dual dessa conjectura para grafos planares pode ser dita como: para todo grafo orientado planar $\vec{G}$ a cintura orientada de $\vec{G}$ é igual a cardinalidade máxima de uma família de transversais duas-a-duas disjuntas. Em outras palavras:

Conjectura 23 (Woodall, 1978). Seja $\vec{G}$ um grafo orientado planar. A cintura de $\vec{G} e ́$ igual ao número máximo de subconjuntos dois-a-dois disjuntos de arcos de $\vec{G}$ tal que a remoção de qualquer um desses subconjuntos destrói todos os circuitos orientados de $\vec{G}$.

Sabemos através de Younger (1998) que nós não podemos remover a hipótese de planaridade dessa versão dual da conjectura de Woodall, pois Thomassen construiu um torneio $T$ com 15 vértices, cintura orientada três e o menor número de arcos que temos de remover para destruir todos os circuitos orientados é maior que um terço do número total de arcos. 
Dessa forma, a cintura orientada de $T$ é maior que a cardinalidade máxima de uma família de transversais duas-a-duas disjuntas, portanto $T$ é um contra-exemplo para tal conjectura.

O torneio de Thomassen é construído da seguinte forma. Ponha $V(T)$ como a união disjunta dos conjuntos

$$
X=\left\{x_{1}, x_{2}, x_{3}, x_{4}, x_{5}\right\}, Y=\left\{y_{1}, y_{2}, y_{3}, y_{4}, y_{5}\right\} \text { e } Z=\left\{z_{1}, z_{2}, z_{3}, z_{4}, z_{5}\right\}
$$

Agora, definimos $E(T)$ primeiro pondo os arcos em cada um dos conjuntos acima $X, Y$ e $Z$ de modo que, a fim de remover todos os circuitos orientados desses subgrafos, devemos ser obrigados a remover pelo menos três arcos de cada subgrafo induzido $T[X], T[Y]$ e $T[Z]$. Isso pode ser feito, por exemplo, tomando os arcos em $X$ sendo a união dos seguintes circuitos orientados: $\left(x_{1}, x_{2}\right)\left(x_{2}, x_{3}\right)\left(x_{3}, x_{1}\right),\left(x_{1}, x_{4}\right)\left(x_{4}, x_{5}\right)\left(x_{5}, x_{1}\right)$ e $\left(x_{2}, x_{5}\right)\left(x_{5}, x_{3}\right)\left(x_{3}, x_{4}\right)\left(x_{4}, x_{2}\right)$.

A esses arcos, acrescentamos os arcos $\left(y_{i}, x_{i}\right),\left(x_{i}, z_{i}\right)$ e $\left(z_{i}, y_{i}\right)$, para cada $i \in\{1,2,3,4,5\}$. Completamos a descrição do conjunto de arcos pondo em $E(T)$ os $\operatorname{arcos}\left(x_{i}, y_{j}\right)$, para cada par $i \neq j \in\{1,2,3,4,5\}$, os $\operatorname{arcos}\left(y_{i}, z_{j}\right)$, para cada par $i \neq j \in\{1,2,3,4,5\}$ e, finalmente, os $\operatorname{arcos}\left(z_{i}, x_{j}\right)$, para cada par $i \neq j \in\{1,2,3,4,5\}$.

Uma tediosa análise de casos mostra que para destruir todos os circuitos orientados de $T$ teremos que remover pelo menos $20+5+5+3+3+3=39>35=105 / 3$ arcos, de um total de 105 arcos. Note que no torneio de Thomassen qualquer subconjunto de $E(T)$ com pelo menos $2 / 3$ dos arcos de $T$ contém um circuito orientado de $T$.

Escrevemos $\vec{G}^{n}$ para um grafo orientado de ordem $n$. O nosso principal resultado é o seguinte.

TeOrema 24. Dados um inteiro $\ell \geqslant 3$ e um real $\beta>0$, para todo $n$ suficientemente grande, existe um grafo orientado $\vec{G}^{n}$ com $O\left(n^{1+1 /(\ell-1)}\right)$ arcos e cintura orientada $\ell$ tal que qualquer $(1 / 2+\beta)$-proporção dos arcos de $\vec{G}^{n}$ contém um circuito orientado de comprimento $\ell$.

Esse resultado é o melhor possível no seguinte sentido: qualquer grafo orientado $\vec{G}$ contém um subgrafo com pelo menos metade de seus arcos e sem circuitos orientados, como pode ser visto tomando-se um ordem total qualquer no conjunto de vértices $V(\vec{G})$. 
O Teorema 24 além de nos dizer que existe uma família infinita de contra-exemplos para o dual da conjectura de Woodall sem a hipótese de planaridade, diz também que os grafos são esparsos, com densidade arbitrariamente pequena.

CoRolÁRIO 25. Para todo $n$ suficientemente grande existe um grafo orientado $\vec{G}^{n}$ com densidade $O\left(n^{-1+1 /(\ell-1)}\right)$ cuja cintura orientada é maior que a cardinalidade máxima de uma familia de transversais duas-a-duas disjuntas.

\section{Um Lema de Regularidade para grafos orientados esparsos}

Nesta seção vamos descrever uma versão do lema de regularidade para grafos orientados esparsos. Antes, precisamos definir alguns conceitos para grafos orientados análogos àqueles que definimos no caso de não haver orientações nas arestas.

Seja $\vec{G}=(V, E)$ um grafo orientado. Para qualquer par de conjuntos disjuntos $U, W \subseteq V$, denotamos o conjunto de arcos e o número de $\operatorname{arcos}$ de $U$ para $W$ por $E_{\vec{G}}(U, W)$ e por $e_{\vec{G}}(U, W)$, isto é

$$
e_{\vec{G}}(U, W)=\left|E_{\vec{G}}(U, W)\right|=\mid\{(a, b) \in E: a \in U \text { e } b \in W\} \mid
$$

respectivamente. Escrevemos $e(U, W)$ e $E(U, W)$ quando o grafo orientado $\vec{G}$ estiver subentendido.

Suponha que sejam dados reais $0<\eta \leqslant 1, D \geqslant 1$ e $0<\cdot p \leqslant 1$. Dizemos que o grafo $\vec{G}$ é $(\eta, D, p)$-esparso se para qualquer par de conjuntos disjuntos $U, W \subseteq V$ com $|U|,|W| \geqslant \eta|V|$, temos que

$$
e_{\vec{G}}(U, W) \leqslant \frac{1}{2} D p|U \| W| .
$$

Definimos a $p$-densidade orientada de $U$ para $W$ em $\vec{G}$ por

$$
d_{\vec{G}, p}(U, W)=\frac{e_{\vec{G}}(U, W)}{(p / 2)|U||W|} .
$$

Dado $\varepsilon>0$, o par $(U, W)$ de conjunto disjuntos não-vazios, com $U, W \subseteq V$, é chamado de $(\varepsilon, \vec{G} ; p)$-regular se para todo $U^{\prime} \subseteq U$, com $\left|U^{\prime}\right| \geqslant \varepsilon|U|$, e todo $W^{\prime} \subseteq W$, com $\left|W^{\prime}\right| \geqslant \varepsilon|W|$, vale que

$$
\left|d_{\vec{G}, p}(U, W)-d_{\vec{G}, p}\left(U^{\prime}, W^{\prime}\right)\right|<\varepsilon .
$$


Dizemos que uma $(\varepsilon, k, \vec{G})$-eqüipartição $\Pi=\left(V_{0}, V_{1}, \ldots, V_{k}\right)$ do conjunto de vértices $V$ é $(\varepsilon, k, \vec{G} ; p)$-regular se $\left|V_{0}\right| \leqslant \varepsilon|V|$ e $\left|V_{i}\right|=\left|V_{j}\right|$ para todo $i, j \in[k]$, e para mais que $(1-\varepsilon)\left(\begin{array}{l}k \\ 2\end{array}\right)$ pares $\{i, j\} \subseteq[k]$, para $i \neq j$, temos que $\left(V_{i}, V_{j}\right)$ e $\left(V_{j}, V_{i}\right)$ são ambos $(\varepsilon, \vec{G} ; p)$-regular.

$\mathrm{Na}$ demonstração do Teorema 24 usamos o seguinte resultado, que é uma versão natural da versão esparsa do Lema de Szemerédi para grafos orientados esparsos.

LEMA 26. Dados os número reais $\varepsilon>0$ e $D \geqslant 1$, e um inteiro $k_{0} \geq 1$, existem constantes $\eta=\eta\left(\varepsilon, k_{0}, D\right)>0$ e $K_{0}=K_{0}\left(\varepsilon, k_{0}, D\right) \geq k_{0}$ tais que para qualquer $0<p=p(n) \leq 1$ vale o seguinte. Todo grafo orientado $(\eta, D, p)$-esparso $\vec{G}$ de ordem suficientemente grande admite uma partição $(\varepsilon, k, \vec{G} ; p)$-regular para algum $k_{0} \leq k \leq K_{0}$.

DemonstraÇÃo. Tomamos uma ordem total qualquer $\prec$ sobre $V(\vec{G})$. Colorimos os arcos de $\vec{G}$ de seguinte forma, se $(v, w) \in E(\vec{G})$ então a cor de $(v, w)$ é azul se $v \prec w$ e vermelha caso contrário. Agora, aplicamos Lema 10, página 43, e temos o resultado acima.

\section{A versão do Lema de Contagem para grafos orientados}

Suponhamos que sejam dados inteiros $m>0$ e $\ell \geqslant 3$, e um vetor de conjuntos dois-a-dois disjuntos $\mathrm{V}^{(m)}=\left(V_{i}\right)_{i=1}^{\ell}$, cada um de cardinalidade $m$. No que segue, os índices dos $V_{i}$ 's serão considerados módulo $\ell$. Sejam $B>0, C \geqslant 1, D \geqslant 1, \varepsilon \leqslant 1, \rho_{0} \leqslant 1$ reais positivos e $M \geqslant 1$ inteiro. Chamamos um grafo orientado $\vec{F}$ de

$$
\left(\varepsilon, \rho_{0}, B, C, D ; \mathbf{V}^{(m)}, M\right)-\text { grafo }
$$

se

(i) $E(\vec{F})=\bigcup_{i=1}^{\ell} E\left(V_{i}, V_{i+1}\right)$ e $|E(\vec{F})|=M$;

(ii) para todo $1 \leqslant i \leqslant \ell$ os pares $\left(V_{i}, V_{i+1}\right)$ são $(\varepsilon, \vec{F} ; \bar{p})$-regulares, para $\bar{p}=B m^{-1+1 /(\ell-1)}$, e a $\bar{p}$-densidade orientada satisfaz

$$
\rho_{0} \leqslant d_{\vec{F}, \bar{p}}\left(V_{i}, V_{i+1}\right) \leqslant D
$$

(iii) para todo $U \subseteq V_{i}$ e todo $W \subseteq V_{i+1}$, para $1 \leqslant i<\ell-1$, tais que

$$
|U| \leqslant|W| \leqslant \frac{1}{2} \bar{p} m|U| \leqslant\left(\frac{1}{2} \bar{p} m\right)^{\ell-2},
$$


temos

$$
e(U, W) \leqslant C|W|
$$

O principal resultado técnico que precisamos é a seguinte versão do lema de contagem do Capítulo 1.

Lema 27. Dado um inteiro $\ell \geq 3$ e dados reais $\sigma>0,0<\alpha \leq 1,0<\rho_{0} \leq 1, C \geq 1 e$ $D \geq 1$ existem constantes positivas $\varepsilon=\varepsilon\left(\ell, \sigma, \alpha, \rho_{0}, C, D\right) \leq 1, B_{0}=B_{0}\left(\ell, \sigma, \alpha, \rho_{0}, C, D\right)>0$, e $m_{0}=m_{0}\left(\ell, \sigma, \alpha, \rho_{0}, C, D\right)$ tais que para qualquer inteiro $m \geq m_{0}$ e $M \geq 1$, e qualquer real $B \geq B_{0}$, o seguinte vale. O número de $\left(\varepsilon, \rho_{0}, B, C, D ; \mathrm{V}^{(m)}, M\right)$-grafos contendo menos que $\sigma m^{\ell /(\ell-1)}$ circuitos de comprimento $\ell$ é no máximo

$$
\alpha^{M}\left(\begin{array}{c}
(\ell+2) m^{2} \\
M
\end{array}\right)
$$

Demonstração. Desconsideramos as orientações dos arcos e aplicamos o Teorema 20, página 55. Retomando as orientações nas arestas, temos que o número de circuitos orientados gerados é igual ao número de circuitos não-orientados.

\section{A demonstração do teorema principal}

Sejam $\ell \geq 3$ e $\beta>0$ dados como nas hipóteses do Teorema 24. Pomos $\delta=\beta / 2$ e definimos as constantes

$$
\rho_{0}=\frac{\delta}{5}, \alpha=\frac{\rho_{0} \ell}{\mathrm{e}^{2}(\ell+2)}, C=4(\ell-1), D=2, \sigma=\frac{1}{2}, \text { e } \gamma=\frac{\delta}{4} .
$$

Sejam $\varepsilon, B_{0}$, e $m_{0}$ as constantes dadas pelo Lema 27 para as quais vale a cota superior (20). Podemos supor que $\varepsilon<\delta / 16$.

Para podermos aplicar o Lema 26, fixamos

$$
k_{0}=\max \left\{\varepsilon^{-1}, s\right\}
$$

onde $s$ é tal que para qualquer inteiro $k \geqslant s$ temos $\operatorname{ex}\left(k, C^{\ell}\right)<(1 / 2+\gamma)\left(\begin{array}{l}k \\ 2\end{array}\right)$. Ressaltamos que quando $\ell$ é par, podemos até omitir o " $1 / 2$ " na definição de $s$, pois $\operatorname{ex}\left(k, C^{\ell}\right)=o\left(k^{2}\right)$ (veja página 29 para a definição de ex $(\cdot, \cdot))$. 
Sejam $\eta=\eta\left(\varepsilon, k_{0}, D\right)$ e $K_{0}=\left(\varepsilon, k_{0}, D\right)$ as constantes dadas pelo Lema 26 para as constantes $\varepsilon, k_{0}$, e $D$ definidas acima. Note que podemos assumir $\eta<\delta / 20$. Fixamos

$$
A=B_{0} K_{0}^{1-1 /(\ell-1)} \quad \text { e } \quad p=A n^{-1+1 /(\ell-1)} .
$$

O Teorema 24 seguirá das duas proposições abaixo que demonstraremos nas subseções finais deste capítulo.

Vamos definir $\mathcal{O G}=\mathcal{O G}(n)$, para todo $n \in \mathbb{N}$, como o conjunto de todos os grafos orienta$\operatorname{dos} \vec{G}^{n}$ sobre o conjunto de vértices $V=V\left(\vec{G}^{n}\right)=[n]$ satisfazendo as seguintes propriedades:

(i) $\vec{G}^{n}$ é $(\eta, 1+\eta, p)$-esparso;

(ii) $e\left(\vec{G}^{n}\right)=(1+o(1))\left(\begin{array}{l}n \\ 2\end{array}\right) p$;

(iii) para todo par de conjuntos disjuntos e não-vazios $U, W \subseteq V$ satisfazendo

$$
|U| \leqslant|W| \leqslant \frac{1}{2} p n|U| \leqslant\left(\frac{1}{2} p n\right)^{\ell-2},
$$

vale o limitante superior no número de $\operatorname{arcos} e(U, W) \leqslant C|W|$ (equação (19)).

Usando o Lema 26 provamos que os grafos em $\mathcal{O G}$ contêm $\left(\varepsilon, \rho_{0}, B, C, D ; \mathrm{V}^{(m)}, M\right)$ subgrafos de um modo muito robusto.

ProposiÇÃo 28. Para qualquer $\vec{G}^{n} \in \mathcal{O G}$, com n suficientemente grande, temos que todo subgrafo $\vec{J} \subseteq \vec{G}^{n}$ com

$$
e(\vec{J}) \geqslant\left(\frac{1}{2}+\delta\right) \frac{n^{2} p}{2}
$$

arcos contém um subgrafo isomorfo a algum

$$
\left(\varepsilon, \rho_{0}, p m^{1-1 /(\ell-1)}, C, D ; \mathrm{V}^{(m)}, M\right) \text {-grafo, }
$$

para algum inteiro $m$, com $n / 2 K_{0} \leqslant m \leqslant n / k_{0}$, e algum inteiro $M \geqslant 1$.

Defina $\mathcal{O} \mathcal{G}^{\prime}$ como o conjunto dos grafos orientados $\vec{G}^{n} \in \mathcal{O G}$ tais que

(iv) todo subgrafo de $\vec{G}^{n}$ isomorfo a algum $\left(\varepsilon, \rho_{0}, B, C, D ; \mathrm{V}^{(m)}, M\right)$-grafo, para $n / 2 K_{0} \leqslant$ $m \leqslant n / k_{0}, B=p m^{1-1 /(\ell-1)}$ e $M \geqslant 1$, contém pelo menos $\sigma_{0} n^{\ell /(\ell-1)}$ circuitos orientados $\vec{C}^{\ell}$, para $\sigma_{0}=\sigma\left(2 K_{0}\right)^{-\ell /(\ell-1)}$; 
(v) o número de circuitos orientados de comprimento no máximo $\ell-1$ em $\vec{G}^{n}$ é no máximo $\bar{A} n^{\ell /(\ell-1)} / \log \log n$, para $\bar{A}=(1 / 16) \max \left\{\ell-1,(\ell-1) A^{\ell-1}\right\}$.

A família $\mathcal{O G}^{\prime}$ não é vazia. De fato, a maioria dos grafos $\vec{G}_{n, p}$ estão em $\mathcal{O G}^{\prime}$.

Proposição 29. Temos $\vec{G}_{n, p} \in \mathcal{O G}^{\prime}$ quase certamente.

Agora, vamos completar a prova do Teorema 24 usando as Proposições 28 e 29. Primeiro, fixamos um grafo $\vec{F}^{n} \in \mathcal{O G}^{\prime}$.

Seja $\vec{G}^{n}$ um grafo obtido de $\vec{F}^{n}$ removendo no máximo $\bar{A} n^{\ell /(\ell-1)} / \log \log n \operatorname{arcos}$, um arco de cada um dos circuitos orientados de comprimento menor que $\ell$ em $\vec{F}^{n}$.

Como pode ser facilmente visto $\vec{G}^{n} \in \mathcal{O G}$. Pela Proposição 28, todo subgrafo $\vec{J} \subseteq \vec{G}^{n}$ com $e(\vec{J}) \geqslant(1 / 2+\beta) e\left(\vec{G}^{n}\right) \geqslant(1 / 2+\delta) n^{2} p / 2$ arcos contém algum $\left(\varepsilon, \rho_{0}, B, C, D ; \mathrm{V}^{(m)}, M\right)$-grafo, para $B=p m^{1-1 /(\ell-1)}$. Como $\vec{G}^{n} \subseteq \vec{F}^{n} \in \mathcal{O G}^{\prime}$ nós temos, pelo item (iv) da definição de $\mathcal{O G}^{\prime}$, que cada $\vec{J}$ como acima contém pelo menos $\left(\sigma_{0}-1 / \log \log n\right) n^{\ell /(\ell-1)}>0$ circuitos orientados de comprimento $\ell$.

Assim, todo subgrafo de $\vec{G}^{n}$ com pelo menos $(1 / 2+\beta) e\left(\vec{G}^{n}\right)$ arcos tem cintura orientada $\ell$; concluindo a prova do Teorema 24.

4.1. Demonstração da Proposição 28. Seja $\vec{J}$ um grafo orientado como dito na Proposição 28. Facilmente, podemos ver que $\vec{J}$ é $(\eta, 1+\eta, p)$-esparso (portanto, $(\eta, D, p)$-esparso).

Seja $\Pi=\left(V_{i}\right)_{i=0}^{k}$ uma partição $(\varepsilon, k, \vec{J} ; p)$-regular dada pelo Lema 26 com as escolhas acima para as constantes $\varepsilon, k_{0}$ e $D$. Ponha $m=\left|V_{i}\right| \leqslant n / k$, para qualquer $i \in[k]$.

Defina o grafo (não-orientado) $R$ cujo conjunto de vértices é $\left\{V_{1}, \ldots, V_{k}\right\}$, e $\left\{V_{i}, V_{j}\right\}$ é uma aresta de $R$ se os pares $\left(V_{i}, V_{j}\right)$ e $\left(V_{j}, V_{i}\right)$ são $(\varepsilon, \vec{J} ; p)$-regulares com as densidades maiores ou iguais a $\rho_{0}$.

Suponha que $e(R)<(1 / 2+\gamma)\left(\begin{array}{c}k \\ 2\end{array}\right)$. Então, como o grafo é $(\eta, 1+\eta, p)$-esparso, o número de arcos em $\vec{J}$ pode ser estimado da seguinte forma

- cada um dos no máximo $\varepsilon\left(\begin{array}{l}k \\ 2\end{array}\right)$ pares irregulares contém no máximo $(1+\eta) p m^{2} \operatorname{arcos}$ e temos, no total,

$$
\leqslant \varepsilon\left(\begin{array}{l}
k \\
2
\end{array}\right)(1+\eta) p m^{2}<\varepsilon(1+\eta) p \frac{n^{2}}{2}
$$


$\operatorname{arcos}$;

- entre os pares $\varepsilon$-regulares com densidade menor que $\rho_{0}$ há menos que $\rho_{0} p m^{2}$ arcos e temos

$$
\leqslant \rho_{0}\left(\begin{array}{l}
k \\
2
\end{array}\right) p m^{2}<\rho_{0}(1+\eta) p \frac{n^{2}}{2}
$$

tais arcos;

- cada $V_{i}$, para $i \in[k]$, contém no máximo (veja as Observações 5 e 6 na página 43)

$$
(1+\eta) p\left(\begin{array}{c}
m \\
2
\end{array}\right)<(1+\eta) p \frac{n^{2}}{k^{2}}
$$

$\operatorname{arcos;}$

- os arcos de $R$ correspondem a $<(1 / 2+\gamma)\left(\begin{array}{c}k \\ 2\end{array}\right)(1+\eta) p m^{2}$ arcos de $\vec{J}$;

- para calcularmos o número de arcos com pelo menos um extremo em $V_{0}$ usamos o seguinte truque. Tomamos $U$ como a união de $V_{1}$ com a classe excepcional $V_{0}$. Assim,

$$
e\left(V_{0}\right)+e\left(V_{0}, V_{1}\right)<e(U) \leqslant(1+\eta) p\left(\begin{array}{c}
m+\varepsilon n \\
2
\end{array}\right),
$$

e o número de arcos que ligam vértices de $V_{0}$ aos vértices das outras classes é

$$
<\sum_{i=2}^{k}(1+\eta) p(m+\varepsilon n)\left|V_{i}\right| .
$$

Portanto, temos no máximo

$$
(1+\eta) p\left(\varepsilon+\frac{1}{k}\right)^{2} \frac{n^{2}}{2}+(1+\eta) p\left(\varepsilon+\frac{1}{k}\right) n^{2}<3\left(\varepsilon+\frac{1}{k}\right)(1+\eta) p \frac{n^{2}}{2}
$$

arcos com um extremo na classe excepcional.

Logo, o número de arcos em $\vec{J}$ é

$$
\begin{aligned}
& e(\vec{J}) \leqslant\left(3 \varepsilon+\frac{3}{k}+\frac{1}{k}+\rho_{0}+\frac{1}{2}+\gamma\right)(1+\eta) \frac{p n^{2}}{2}<\left(4 \varepsilon+\frac{4}{k}+\rho_{0}+\frac{1}{2}+\gamma+\eta\right) \frac{p n^{2}}{2}< \\
& <\left(\frac{1}{2}+\frac{19}{20} \delta\right) \frac{p n^{2}}{2}
\end{aligned}
$$

contradizendo (23).

Portanto, $R$ contém pelo menos $(1 / 2+\gamma)\left(\begin{array}{c}k \\ 2\end{array}\right)$ arcos e, pela escolha de $k_{0}$ em (21), podemos concluir que $R$ contém um circuito $V_{i_{1}}, \ldots, V_{i_{\ell}}$. 
Assim, temos um $\left(\varepsilon, \rho_{0}, p m^{1-1 /(\ell-1)}, C, D ; \mathrm{V}^{(m)}, M\right)$-grafo dado por: o conjunto de vértices $\mathrm{V}^{(m)}$ é o vetor $\left(V_{i_{1}}, \ldots, V_{i_{\ell}}\right)$ de $m$-subconjuntos de $V$ dois-a-dois disjuntos, o conjunto de arcos é $\bigcup_{j=1}^{\ell} E_{\vec{J}}\left(V_{i_{j}}, V_{i_{j+1}}\right)$ (os índices são tomados módulo $\ell$ ) e $M$ é a cardinalidade de tal união. Para essas escolhas de $\mathbf{V}^{(m)}$ e $M$, e as escolhas acima para $\varepsilon, \rho_{0}, B, C$ e $D$ podemos, facilmente, verificar os itens (i)-(iii) da definição de um $\left(\varepsilon, \rho_{0}, B, C, D ; \mathrm{V}^{(m)}, M\right)$-grafo. Isso completa a prova da Proposição 28.

4.2. Demonstração da Proposição 29. Agora, vamos provar que $\vec{G}_{n, p}$, com a escolha acima para $p$, satisfaz os itens (i)-(iii) da definição de $\mathcal{O G}$ e satisfaz os itens (iv) e (v) de definição de $\mathcal{O G}^{\prime}$ quase certamente.

Para verificarmos que o item (i) vale com alta probabilidade, observamos que, pela desigualdade de Chernoff (equação 8 na página 33) para qualquer par de conjuntos disjuntos $U$, $W \subseteq V \operatorname{com}|U|,|W| \geqslant \eta n$, temos

$$
\begin{aligned}
\mathbb{P}\left\{e(U, W) \geqslant(1+\eta) \frac{p}{2}|U||W|\right\} & \leqslant \exp \left\{-\frac{\eta^{2}((p / 2)|U||W|)^{2}}{2(1+(\eta / 3))((p / 2)|U||W|)}\right\} \\
& <\exp \left\{-\frac{1}{3} \eta^{2} \frac{p}{2}|U||W|\right\} \\
& \leqslant \exp \left\{\frac{A}{6} \eta^{4} n^{1+1 /(\ell-1)}\right\} .
\end{aligned}
$$

Então, o número esperado de pares $U, W \subseteq V$ com $|U|,|W| \geqslant \eta n$ e que violam a condição de $(\eta, 1+\eta, p)$-esparsidade é no máximo $4^{n} \exp \left\{-A 6^{-1} \eta^{4} n^{1+1 /(\ell-1)}\right\}=o(1)$. Assim, se $X$ é o número de pares de conjuntos $U, W \subseteq V$ com pelo menos $\eta n$ vértices e que violam a condição de $(\eta, 1+\eta, p)$-esparsidade, temos, pela desigualdade de Markov, que

$$
\mathbb{P}\{X>0\}<4^{n} \exp \left\{-A 6^{-1} \eta^{4} n^{1+1 /(\ell-1)}\right\}=o(1),
$$

ou seja, quase certamente $X=0$.

Obtemos o item (ii) com alta probabilidade também a partir da desigualdade de Chernoff. Ou seja, para todo real $\xi>0$, a probabilidade que $\left|e\left(\vec{G}_{n, p}\right)-p\left(\begin{array}{c}n \\ 2\end{array}\right)\right|>\xi p\left(\begin{array}{c}n \\ 2\end{array}\right)$ é exponencialmente pequena em $p n^{2}$.

Para provar que as propriedades (iii) e (iv) valem para $\vec{G}_{n, p}$ quase certamente, novamente aplicamos a desigualdade de Markov a uma variável aleatória apropriada. Vamos considerar 
primeiro o item (iii). Seja $U, W \subseteq V$ subconjuntos disjuntos satisfazendo (22). Para verificarmos que (iii) vale com alta probabilidade, observemos que a probabilidade com que (19) falha é

$$
\mathbb{P}\{e(U, W)>C|W|\} \leqslant\left(\begin{array}{c}
|U||W| \\
C|W|
\end{array}\right)\left(\frac{p}{2}\right)^{C|W|} \leqslant\left(\frac{\mathrm{e}}{C} \cdot \frac{p|U|}{2}\right)^{C|W|} .
$$

Note que $(1 / 2) p|U| \leqslant((1 / 2) p n)^{\ell-2} / n \leqslant(A / 2)^{\ell-2} n^{-1 /(\ell-1)}$. De $|U| \leqslant|W|<\lambda n$, para todo $\lambda>0$, concluímos que $\left(\begin{array}{c}n \\ |U|\end{array}\right) \leqslant\left(\begin{array}{c}n \\ |W|\end{array}\right)$ e, portanto, $\left(\begin{array}{c}n \\ |U|\end{array}\right)\left(\begin{array}{c}n \\ |W|\end{array}\right) \leqslant\left(\begin{array}{c}n \\ |W|\end{array}\right)^{2}$. Essas desigualdades implicam que o número esperado de subconjuntos $U$ e $W$, com cardinalidades $u$ e $w$, respectivamente, para os quais (22) vale e (19) falha, é no máximo

$$
\begin{aligned}
\sum_{1 \leqslant w<\lambda n} \sum_{u=1}^{w}\left(\begin{array}{l}
n \\
u
\end{array}\right)\left(\begin{array}{l}
n \\
w
\end{array}\right)\left(\frac{\mathrm{e}}{C} \cdot \frac{p u}{2}\right)^{C w} \leqslant \sum_{1 \leqslant w<\lambda n} w\left(\begin{array}{c}
n \\
w
\end{array}\right)^{2}\left(\frac{\mathrm{e}}{C}\left(\frac{A}{2}\right)^{\ell-2} n^{-1 /(\ell-1)}\right)^{C w} \\
\leqslant \sum_{1 \leqslant w<\lambda n} w\left(\frac{\mathrm{e} n}{w}\right)^{2 w}\left(\frac{\mathrm{e}}{C}\left(\frac{A}{2}\right)^{\ell-2} n^{-1 /(\ell-1)}\right)^{C w} \\
=\sum_{1 \leqslant w<\lambda n} \frac{w}{w^{2 w}}\left(\frac{\mathrm{e}^{1+2 / C}}{C}\left(\frac{A}{2}\right)^{\ell-2} n^{(2 / C)-(1 /(\ell-1))}\right)^{C w} \\
=\sum_{1 \leqslant w<\lambda n} \frac{w}{w^{2 w}}\left(\frac{\mathrm{e}^{1+2 / C}}{C}\left(\frac{A}{2}\right)^{\ell-2} n^{-2 / C}\right)^{C w} \\
=\sum_{1 \leqslant w<\lambda n} o(1)^{C w} \\
=o(1)
\end{aligned}
$$

para $C=4(\ell-1)$ e qualquer $\lambda>0$ fixo. Assim, temos (iii) com probabilidade $1-o(1)$.

Para verificarmos o item (iv), notamos que $K_{0} \geqslant n / m$, e então

$$
B=p m^{1-1 /(\ell-1)} \geqslant B_{0}\left(K_{0} m / n\right)^{1-1 /(\ell-1)} \geqslant B_{0}
$$

$\mathrm{O}$ número de $\operatorname{arcos} M$ é pelo menos $\rho_{0}(p / 2) m^{2} \ell$. Fixados inteiros positivos $m$ e $M \geqslant$ $\rho_{0}(p / 2) m^{2} \ell$, o número esperado de $\left(\varepsilon, \rho_{0}, B, C, D ; \mathrm{V}^{(m)}, M\right)$-subgrafos de $\vec{G}_{n, p}$ contendo no 
máximo $\sigma_{0} n^{\ell /(\ell-1)} \leqslant \sigma m^{\ell /(\ell-1)}$ circuitos $\vec{C}^{\ell}$ é, pelo Lema 27 , no máximo

$$
\begin{aligned}
(n)_{\ell m} \alpha^{M}\left(\begin{array}{c}
(\ell+2) m^{2} \\
M
\end{array}\right)\left(\frac{p}{2}\right)^{M} & \leqslant n^{\ell m}\left(\alpha \cdot \frac{\mathrm{e}(\ell+2) m^{2}}{M} \cdot \frac{p}{2}\right)^{M} \\
& \leqslant n^{\ell m}\left(\frac{\mathrm{e} \alpha(\ell+2)}{\ell \rho_{0}}\right)^{M} \\
& =n^{\ell m}\left(\frac{1}{\mathrm{e}}\right)^{M} \\
& \leqslant \exp \left\{(\log n) m \ell-\rho_{0}(p / 2) m^{2} \ell\right\} \\
& =o\left(n^{-3}\right) .
\end{aligned}
$$

Somando sobre todas as possíveis escolhas para $m \in[n]$ e $M \in\left[\left(\begin{array}{c}n \\ 2\end{array}\right)\right]$, temos um fator adicional de no máximo $n^{3}$. Portanto, pela desigualdade de Markov, a propriedade (iv) vale com probabilidade $1-o(1)$.

Finalmente, o número esperado de circuitos orientados curtos é

$$
\sum_{i=3}^{\ell-1} \frac{(n)_{i}}{2 i}\left(\frac{p}{2}\right)^{i} \leqslant \frac{1}{16} \sum_{i=3}^{\ell-1} n^{i} p^{i} \leqslant \bar{A} n,
$$

e pela desigualdade de Markov, vemos que a probabilidade de o número de circuitos curtos ser maior que $\bar{A} n^{\ell /(\ell-1)} / \log \log n$ é menor que $\log \log n / n^{1 /(\ell-1)}=o(1)$.

4.3. Demonstração do Corolário 25. Seja $\ell \geqslant 3$ um inteiro. Ponha $\beta=1 / 2-1 / \ell$ e considere $\vec{G}^{n}$ um grafo orientado de cintura orientada $\ell$ dado pelo Teorema 24 .

Suponha que temos em $\vec{G}^{n}$ uma família $\mathcal{T}$ de transversais duas-a-duas disjuntas de cardinalidade $|\mathcal{T}|=\ell$. Seja $\vec{H}$ o grafo obtido de $\vec{G}^{n}$ removendo uma transversal $T \in \mathcal{T}$ desta família, onde $|T| \leqslant e\left(\vec{G}^{n}\right) / \ell$. Temos que $e(\vec{H}) \geqslant(1-1 / \ell) e\left(\vec{G}^{n}\right)=(1 / 2+\beta) e\left(\vec{G}^{n}\right)$ e, pelo Teorema 24, o grafo orientado $\vec{H}$ deve conter um circuito orientado, contradizendo o fato de $T$ ser uma transversal.

\section{Considerações finais}

Ambos resultados deste capítulo, Teorema 24 e Corolário 25, afirmam a existência de grafos orientados esparsos de cintura orientada $\ell$, que contêm circuitos orientados de comprimento $\ell$ de um modo bastante robusto. Nossa técnica de demonstração é não-construtiva. É interessante saber se seríamos capazes de provar esses resultados construtivamente. 
Aparentemente (Younger, 1998) não é conhecido um método para verificar o contraexemplo de Thomassen que não seja a força-bruta.

Finalmente, e mais importante, o caso geral da conjectura de Woodall e o dual para grafos planares não foram resolvidos até o momento. 


\section{CAPíTULO 3}

\section{GRAFOS $\left(C^{\ell}, H\right)$-RAMSEY MINIMAIS}

Lembramos que $\Gamma \rightarrow(G, H)$ significa que, não importa como colorimos as arestas do grafo $\Gamma$ com duas cores, digamos vermelha e azul, sempre ocorre em $\Gamma$, ou uma cópia vermelha do grafo $G$, ou uma cópia azul do grafo $H$. Quando $\Gamma \rightarrow(G, H)$ nós dizemos que $\Gamma$ é ramsey com relação ao par $(G, H)$.

Dizemos que o grafo $\Gamma$ é ramsey-crítico com relação ao par de grafos $(G, H)$ se ele for ramsey com relação a esse par, isto é $\Gamma \rightarrow(G, H)$, mas nenhum subgrafo próprio o é, ou seja $\Gamma^{\prime} \nrightarrow(G, H)$ para todo subgrafo próprio $\Gamma^{\prime} \subset \Gamma$. Além disso, dizemos que o par $(G, H)$ é ramsey-finito se o conjunto dos grafos ramsey-críticos com relação ao par $(G, H)$ for finito. Caso contrário, dizemos que $(G, H)$ é ramsey-infinito.

O nosso objetivo é demonstrar o seguinte resultado.

Teorema 30. Dado um inteiro $\ell \geqslant 4$, se $H$ é 2-conexo e não contém circuito induzido de comprimento pelo menos $\ell$, então o par $\left(C^{k}, H\right)$ é ramsey-infinito para todo $k \geqslant \ell$.

\section{Introdução}

O problema de caracterizar os pares de grafos para os quais existem infinitos grafos ramseycríticos foi proposto por Nešetřil e Rödl (1976) e tem sido respondido para vários pares de grafos.

Entre outros resultados, Nešetřil e Rödl (1978) provaram que o par $(G, H)$ é ramseyinfinito nos seguintes três casos: (i) $G$ e $H$ são florestas que contêm um caminho de comprimento pelo menos três, (ii) $G$ e $H$ têm número cromático pelo menos três e (iii) $G$ e $H$ são 3-conexos. Ainda, Burr (1986) provou que se $G$ é 2-conexo e tal que a remoção de quaisquer dois vértices não-adjacentes não desconecta o grafo, então o par $(G, G)$ é ramsey-infinito. 
Quando pelo menos um dos grafos envolvidos é uma estrela ou um emparelhamento, nós conhecemos os seguintes resultados. Burr et al. (1978) provam que se $G$ é um emparelhamento, então o par $(G, H)$ é ramsey-finito para qualquer que seja o grafo $H$. A partir de um teorema provado por Euczak (1994) e de outros resultados conhecidos, sabemos o seguinte: (i) quando $H$ é uma estrela de ordem ímpar, ou (ii) quando $H$ é uma floresta que contêm um caminho de comprimento pelo menos três, o par $(G, H)$ é ramsey-finito se, e somente se, $G$ é um emparelhamento. Esse dois resultados seguem do seguinte teorema de Euczak (1994), citado logo acima: se $G$ é uma floresta que não é um emparelhamento e $H$ contém pelo menos um circuito, então o par $(G, H)$ é ramsey-infinito. Esse resultado de Luczak caminha no sentido da seguinte conjectura proposta por Burr.

Conjectura 31 (Burr et al., 1980). Se o par $(G, H)$ é ramsey-finito para todo $H$, então G é um emparelhamento.

Um resultado de Faudree (1991) basicamente caracteriza todos pares ramsey-finitos formados por duas florestas.

Alguns resultados recentes, além dos resultados de Euczak acima, são: uma aplicação direta do Teorema 6 de Rödl e Ruciński (1993) e do Corolário 4 de Rödl e Ruciński (1995) nos mostra que $(G, G)$ é ramsey-infinito para todo grafo $G$ que contém circuito. Kohayakawa e Kreuter (1997) provam que o par formado por circuitos $\left(C^{\ell_{1}}, C^{\ell_{2}}\right)$ é ramsey-infinito. Nesses dois últimos resultados o Lema de Regularidade foi usado como ferramenta.

Uma importante conjectura foi proposta em 1980:

Conjectura 32 (Burr et al., 1981b). O par $(G, H)$ é ramsey-infinito a menos que ambos os grafos sejam estrelas de ordem par ou pelo menos um dos grafos contém um $K^{2}$ como componente conexa.

Faudree et al. (1997) observam que "um caso interessante da conjectura acima é quando $G$ é um circuito e $H$ é 2-conexo. Até o momento nenhuma técnica é conhecida para provar que tal par é ramsey-infinito." Nosso resultado diz que o par $(G, H)$ é ramsey-infinito para $G$ circuito e $H$ numa subclasse da classe dos grafos 2-conexo. De fato, provaremos o Teorema 33 abaixo que claramente implica o Teorema 30. Primeiro, vamos introduzir alguma notação. 
Dado um inteiro $t>0$, escrevemos $\Gamma \dashv_{t}(G, H)$ se, para todo $U \subset V(\Gamma)$ com $|U| \leqslant t$, vale que $\Gamma[U] \nrightarrow \rightarrow(G, H)$, ou seja, existe uma vermelho-azul aresta-coloração de $\Gamma[U]$ tal que não ocorre em $\Gamma[U]$, nem cópia vermelha de $G$, nem cópia azul de $H$.

Para qualquer inteiro $\ell \geqslant 4$, escrevemos $\mathcal{C}(\ell)$ para a família dos grafos 2-conexos que não contêm circuitos induzidos de comprimento maior ou igual a $\ell$. O resultado que demonstramos na Seção 3 é o seguinte.

TEOREma 33. Dados inteiros $\ell \geqslant 4$ e $t \geqslant 1$ e um grafo $H \in \mathcal{C}(\ell)$, existe um grafo $\Gamma$ tal que $\Gamma \rightarrow\left(C^{\ell}, H\right)$, mas $\Gamma \nrightarrow_{t}\left(C^{\ell}, H\right)$.

Ou seja, para todo $t \in \mathbb{N}$, existe um grafo $\Gamma$ tal que $\Gamma \rightarrow\left(C^{\ell}, H\right)$ e todo subgrafo de $\Gamma$ que é minimal com respeito a propriedade " $\rightarrow\left(C^{\ell}, H\right)$ " deve ter mais que $t$ vértices. Isso nos leva a concluir a afirmação do Teorema 30.

Ademais, no que segue sempre estaremos considerando $n$ grande suficiente para as desigualdades valerem.

1.1. A estratégia da demonstração do Teorema 33. Agora, passamos a descrever informalmente a demonstração do Teorema 33 .

Seja $H$ um grafo em $\mathcal{C}(\ell)$ com $h$ vértices, onde $\ell \geqslant 4$. Para começar, escolhemos uma constante grande $A>0$ e tomamos um hipergrafo $h$-uniforme aleatório $\mathcal{H}_{n, p_{H}}$ escolhendo, de acordo com o modelo binomial, cada elemento de $\left(\begin{array}{c}{[n]} \\ h\end{array}\right)$ com probabilidade $p_{H}=A n^{-(h-1)+1 /(\ell-1)}$. Para essa escolha de $p_{H}$, podemos verificar que cada elemento de $E\left(\mathcal{H}_{n, p_{H}}\right)$ quase certamente pertence a uma quantidade constante e grande de hipercircuitos de comprimento $\ell$. Também, podemos verificar que quase certamente o número de hipercircuitos de comprimento menor que $\ell$ em $\mathcal{H}_{n, p_{H}}$ é $o\left(\left|E\left(\mathcal{H}_{n, p_{H}}\right)\right|\right)$.

Agora, obtemos um hipergrafo linear $\mathcal{G}$ de cintura $\ell$ removendo de $\mathcal{H}_{n, p_{H}}$ uma hiperaresta de cada um dos $o\left(\left|E\left(\mathcal{H}_{n, p_{H}}\right)\right|\right)$ hipercircuitos de comprimento menor que $\ell$. A partir disso, definimos o grafo $\Gamma=\Gamma^{n}$ mergulhando em cada hiperaresta de $\mathcal{G}$ uma cópia do grafo $H$. O fato do grafo $H$ pertencer à família $\mathcal{C}(\ell)$ implica que todas as cópias de $H$ contidas no grafo $\Gamma$ são aquelas que foram mergulhadas nas hiperarestas de $\mathcal{G}$. Em particular, todas as cópias de $H$ em $\Gamma$ são induzidas. 
Afirmamos que tal grafo $\Gamma$ satisfaz a tese do Teorema 33. Primeiro, queremos provar que qualquer aresta-coloração com as cores vermelha e azul do grafo $\Gamma$ resulta ou numa cópia vermelha de $C^{\ell}$ ou uma cópia azul de $H$. Suponha que um inimigo tenha pintado as arestas de $\Gamma$. Então, nosso adversário pintou pelo menos uma arestas de cada cópia de $H \subset \Gamma$ de vermelho. Lembramos que as únicas cópias de $H$ são aquelas que foram mergulhadas em $\mathcal{G}$, logo podemos supor que o inimigo escolheu exatamente uma aresta de cada cópia de $H$ e, assim, destruiu qualquer chance de termos uma cópia azul de $H$. Então, queremos mostrar que essas arestas vermelhas criam uma cópia de $C^{\ell}$ em $\Gamma$.

Denotamos por $\Gamma^{(e)}$ o subgrafo gerador de $\Gamma$ cujas arestas são essas que foram pintadas de vermelho. Note que $\Gamma^{(e)}$ tem $|E(\mathcal{G})|=(1+o(1))\left|E\left(\mathcal{H}_{n, p_{H}}\right)\right| \asymp n^{1+1 /(\ell-1)}$ arestas, pois cada aresta de $\Gamma$ está contida numa única cópia de $H$ em $\Gamma$. Além disso, como mostraremos adiante, existe uma constante $D \geqslant 1$ para a qual o grafo $\Gamma^{(e)}$ é $\left(\eta, D, p_{e}\right)$-esparso, com $p_{e}=$ $A n^{-1+1 /(\ell-1)}$ e $\eta$ arbitrariamente pequeno.

Observamos, embora não seja diretamente relevante para a demonstração, que o grafo aleatório $G_{n, p_{e}}$ quase certamente contém tantas arestas quantos circuitos de comprimento $\ell$, mais que isso, quase certamente cada aresta de $G_{n, p_{e}}$ pertence a um grande número de circuitos de comprimento $\ell$. Também, pelo Teorema 3 na página 22 , quase certamente $G_{n, p_{e}} \rightarrow\left(C^{\ell}, C^{\ell}\right)$ se $A>C$, onde $C$ é a constante dada por esse teorema.

Como o grafo $\Gamma^{(e)}$ é $\left(\eta, D, p_{e}\right)$-esparso podemos aplicar a versão esparsa do Lema de Regularidade, com uma escolha apropriada para $k_{0}$ e $\varepsilon$, a fim de obtermos uma $\ell$-upla $\left(V_{1}, \ldots, V_{\ell}\right)$ de subconjuntos de $V(\Gamma)$ tais que $\left(V_{i}, V_{i+1}\right)$ é $\left(\varepsilon ; p_{e}\right)$-regular com $p_{e}$-densidade positiva, para todo $i \in[\ell]$, onde os índices são considerados módulo $\ell$. Pelo Lema de Contagem do Capítulo 1 quase certamente $\Gamma$ é tal que essa $\ell$-upla de subconjuntos de $V(\Gamma)$ induz um circuito $C^{\ell}$ em $\Gamma^{(e)}$. Logo $\Gamma \rightarrow\left(C^{\ell}, H\right)$.

Para mostrarmos que $\Gamma t_{t}\left(C^{\ell}, H\right)$, para algum $t>0$ fixo, usamos o fato de que quase certamente $\Gamma$ é tal que, para todo $U \subset V(\Gamma)$ com $|U| \leqslant t$, o grafo $\Gamma[U]$ contém um vértice que pertence a no máximo uma cópia de $H$ que está totalmente contida em $U$. Com isso, conseguimos colorir indutivamente as arestas de $\Gamma[U]$ de modo que $\Gamma[U] \nrightarrow \rightarrow\left(C^{\ell}, H\right)$. 


\section{A construção do grafo $\Gamma=\Gamma^{n}(\ell, t, H)$}

Nesta seção, vamos introduzir a definição do grafo $\Gamma$ e mostrar suas propriedades relevantes na demonstração do Teorema 33.

2.1. A construção. Suponhamos que são dados inteiros $\ell \geqslant 4, t \geqslant 1$ e um grafo $H \in$ $\mathcal{C}(\ell)$. Vamos fixar $V(\Gamma)=[n]$ e definir

$$
p_{H}=p_{H}(n)=A n^{-(h-1)+1 /(\ell-1)},
$$

para uma constante $A$ positiva a ser escolhida adiante. Tomamos sobre $[n]$ o hipergrafo $h$ uniforme aleatório $\mathcal{H}_{n, p_{H}}$, segundo o modelo binomial padrão. O número esperado de hiperarestas em $\mathcal{H}_{n, p_{H}}$ é

$$
\mathbb{E} e\left(\mathcal{H}_{n, p_{H}}\right)=\left(\begin{array}{l}
n \\
h
\end{array}\right) p_{H}=\frac{(n)_{h}}{n^{h}} \frac{A}{h !} n^{1+1 /(\ell-1)}
$$

ou seja, para todo $\xi>0$ vale que

$$
(1-\xi) \frac{A}{h !} n^{1+1 /(\ell-1)}<\mathbb{E} e\left(\mathcal{H}_{n, p_{H}}\right)<\frac{A}{h !} n^{1+1 /(\ell-1)},
$$

se $n$ é suficientemente grande. Pela desigualdade de Chernoff (equação (8), página 33)

$$
(1-\xi)^{2} \frac{A}{h !} n^{1+1 /(\ell-1)}<e\left(\mathcal{H}_{n, p_{H}}\right)<(1+\xi) \frac{A}{h !} n^{1+1 /(\ell-1)}
$$

com probabilidade tendendo a 1 , quando $n \rightarrow \infty$, para todo $\xi>0$. Ou seja, quase certamente, o número de arestas em $\mathcal{H}_{n, p_{H}}$ é

$$
(1+o(1))(A / h !) n^{1+1 /(\ell-1)}
$$

Definimos o hipergrafo $\mathcal{G}$, linear e de cintura $\ell$, removendo de $\mathcal{H}_{n, p_{H}}$ uma hiperaresta de cada hipercircuito de comprimento $k$, para todo $2 \leqslant k<\ell$. Observamos que o número esperado de hipercircuitos de comprimento $k$ em $\mathcal{H}_{n, p_{H}}$ é no máximo,

$$
c(h, k)\left(\begin{array}{l}
n \\
k
\end{array}\right)\left(\begin{array}{c}
n-k \\
k(h-2)
\end{array}\right) p_{H}{ }^{k} \asymp n^{k /(\ell-1)} \leqslant n=o\left(e\left(\mathcal{H}_{n, p_{H}}\right)\right),
$$

para todo $2 \leqslant k<\ell$, sendo $c(h, k)$ uma constante que depende só de $h$ e $k$. Pela desigualdade de Chebyshev temos que o número de hipercircuitos curtos não desvia muito do seu valor esperado. 
Vejamos essa última afirmação. Fixamos dois hipercircuitos com $k$ arestas do hipergrafo $h$-uniforme completo sobre $[n]$. Suponha que esses dois hipercircuitos compartilham $j$ arestas, para algum $1 \leqslant j \leqslant k-1$. Então, a probabilidade de ambos estarem presentes em $\mathcal{H}_{n, p_{H}}$ é $p_{H}{ }^{2 k-j}$. O valor esperado para o número de pares de hipercircuitos de comprimento $k$ que compartilham $j$ arestas é no máximo

$$
n^{(h-1)(2 k-j)-1} p_{H}^{2 k-j} \leqslant n^{-1+(2 k-j) /(\ell-1)} .
$$

Assim, se $X_{k}\left(\mathcal{H}_{n, p_{H}}\right)$ é o número de hipercircuitos de comprimento $k$ em $\mathcal{H}_{n, p_{H}}$, onde $2 \leqslant k<$ $\ell$, temos que

$$
\operatorname{Var}\left(X_{k}\right) \leqslant \mathbb{E} X_{k}+\sum_{j=1}^{k-1} n^{-1+(2 k-j) /(\ell-1)} .
$$

Usando a desigualdade de Chebyshev, equação (6) na página 32, para todo $\lambda>0$ temos

$$
\mathbb{P}\left\{\left|X_{k}-\mathbb{E} X_{k}\right| \geqslant \lambda \mathbb{E} X_{k}\right\} \leqslant \frac{\operatorname{Var}\left(X_{k}\right)}{\lambda^{2} \mathbb{E} X_{k}^{2}} \leqslant \frac{1}{\lambda^{2} n^{2 k /(\ell-1)}}+\sum_{j=1}^{k-1} \frac{n^{-1+(2 k-j) /(\ell-1)}}{\lambda^{2} n^{2 k /(\ell-1)}}=o(1) .
$$

Ou seja, para cada $2 \leqslant k<\ell$ o número de hipercircuitos de comprimento $k$ em $\mathcal{H}_{n, p_{H}}$ está concentrado em um intervalo de tamanho $2 \lambda$ em torno do valor esperado, para todo $\lambda>0$. Portanto, temos que

$$
e(\mathcal{G})=(1+o(1)) e\left(\mathcal{H}_{n, p_{H}}\right)
$$

Agora, em cada hiperaresta $F \in E(\mathcal{G})$ nós mergulhamos uma cópia do grafo $H$ arbitrariamente, ou seja, para cada hiperaresta $F$ de $\mathcal{G}$ nós tomamos isomorfismos $\iota_{F}: V(H) \hookrightarrow F$ arbitrários.

Finalmente, definimos o grafo $\Gamma=(V, E)$ pondo $V=[n] \mathrm{e}$

$$
E=\bigcup_{F \in E(\mathcal{G})}\left\{\left\{\iota_{F}(x), \iota_{F}(y)\right\}:\{x, y\} \in E(H)\right\}
$$

Adiante, será importante distinguirmos entre dois tipos de cópia de $H$ em $\Gamma$ : os subgrafos $\iota_{F}(H) \subset \Gamma$, para todo $F \in E(\mathcal{G})$, são chamados cópias não-espontâneas de $H$ em $\Gamma$. Qualquer outra cópia de $H$ em $\Gamma$ é chamada de cópia espontânea. 
2.2. Subgrafos de $\Gamma$. Vamos escrever $\Gamma^{(e)}$ para um subgrafo gerador de $\Gamma$ tal que arestas distintas de $\Gamma^{(e)}$ podem ser estendidas para cópias não-espontâneas distintas de $H \mathrm{em} \Gamma$. Isso significa que a função que associa cada $f \in E\left(\Gamma^{(e)}\right)$ a uma hiperaresta $F_{f} \in E(\mathcal{G})$ é injetiva.

Seja $J$ um grafo sobre $[n]$ com conjunto de arestas $\left\{e_{1}, \ldots, e_{M}\right\} \subset\left(\begin{array}{c}n n \\ 2\end{array}\right)$. Agora, queremos estimar a probabilidade com que $\Gamma$ contém um subgrafo $\Gamma^{(e)}$ tal que $J \subseteq \Gamma^{(e)}$.

Suponha que para algum $\Gamma^{(e)} \subset \Gamma$ tenhamos $J \subseteq \Gamma^{(e)}$. Então, existe uma $M$-upla de hiperarestas

$$
\left(E_{1}, \ldots, E_{M}\right) \in E(\mathcal{G}) \times \cdots \times E(\mathcal{G})
$$

tal que $e_{i} \subset E_{i}$ e $E_{i} \neq E_{j}$ para todo $i, j \in[M]$, com $i \neq j$.

Se $X\left(\mathcal{H}_{n, p_{H}}\right)$ é o número de tais $M$-uplas em $\mathcal{H}_{n, p_{H}}$, então

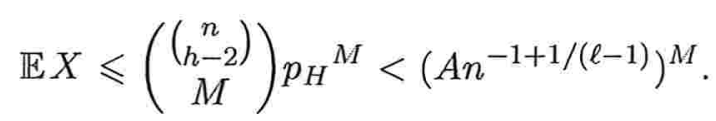

Assim, a probabilidade de termos $J \subseteq \Gamma^{(e)}$, para algum $\Gamma^{(e)} \subset \Gamma$, é menor que a probabilidade de existir uma $M$-upla de hiperarestas como na equação (27), e pela desigualdade de Markov, temos

$$
\mathbb{P}\left\{\exists \Gamma^{(e)} \subset \Gamma: J \subseteq \Gamma^{(e)}\right\}<p_{e}{ }^{M}, \text { onde } p_{e}=A n^{-1+1 /(\ell-1)} .
$$

2.2.1. Subgrafos bipartidos de $\Gamma$. Agora, vamos estudar propriedades de subgrafos bipartidos do grafo $\Gamma$. O primeiro resultado diz que subgrafos bipartidos grandes não induzem muitas arestas, não muito mais que o esperado. O segundo resultado diz respeito ao número de arestas em grafos bipartidos pequenos.

ProposiçÃo 34. Quase certamente o grafo $\Gamma$ é $\left(\eta, \mathrm{e} 2 e(H), p_{e}\right)$-esparso para todo $\eta$ positivo.

Demonstração. Suponha que existam $U, W \subseteq[n]$ disjuntos com $|U|=|W|=\lceil\eta n\rceil$ e tais que $e_{\Gamma}(U, W)>\mathrm{e} 2 e(H) p_{e}|U||W|$. Então, temos que

$$
e_{\Gamma^{(e)}}(U, W) \geqslant M=\left\lceil\mathrm{e} 2 p_{e}|U||W|\right\rceil
$$


para algum $\Gamma^{(e)} \subseteq \Gamma$, e esse evento ocorre com probabilidade no máximo

$$
\left(\begin{array}{c}
|U||W| \\
M
\end{array}\right) p_{e}{ }^{M} \leqslant\left(\frac{\mathrm{e}|U||W|}{M} p_{e}\right)^{M} \leqslant\left(\frac{1}{2}\right)^{M}
$$

O número de conjuntos $U, W \subseteq V$ disjuntos e de cardinalidade $\lceil\eta n\rceil$ é menor que $2^{2 n}$. Então, o número esperado de subconjuntos disjuntos $U, W$ induzindo pelo menos $M$ arestas em $\Gamma^{(e)}$ é no máximo $2^{2 n-M}$. Pela desigualdade de Markov, se $X$ conta o número de pares de conjuntos $U$ e $W$ que induzem muitas arestas, então

$$
\mathbb{P}\{X>0\} \leqslant \mathbb{E} X=2^{2 n-M}=o(1),
$$

pois $M=\Omega\left(n^{1+1 /(\ell-1)}\right)$. Ou seja, $X=0$ com probabilidade tendendo a 1 conforme $n \rightarrow$ $\infty$.

Dizemos que um grafo de ordem $n$ é $\left(p_{e}, C, \ell\right)$-localmente-esparso se para quaisquer subconjuntos disjuntos de vértices $U$ e $W \operatorname{com}|U| \leqslant|W| \leqslant\left(p_{e} n\right)|U| \leqslant\left(p_{e} n\right)^{\ell-2}$ vale que $e(U, W) \leqslant C|W|$.

Proposição 35. Quase certamente o grafo $\Gamma$ é $\left(p_{e}, 4(\ell-1) e(H), \ell\right)$-localmente-esparso.

Demonstração. Escrevemos $C=4(\ell-1) e(H)$ e $C^{\prime}=C / e(H)=4(\ell-1)$. Como na prova da proposição anterior, se $e_{\Gamma}(U, W)>C|W|$, então existe $\Gamma^{(e)} \subset \Gamma \operatorname{com} e_{\Gamma(e)}(U, W)>C^{\prime}|W|$. Fixados $U$ e $W$, a probabilidade desse par induzir um subgrafo bipartido com muitas arestas em algum $\Gamma^{(e)}$ é

$$
\mathbb{P}\left\{\exists \Gamma^{(e)} \subset \Gamma: e_{\Gamma^{(e)}}(U, W)>C^{\prime}|W|\right\} \leqslant\left(\begin{array}{c}
|U||W| \\
\left\lceil C^{\prime}|W|\right\rceil
\end{array}\right) p_{e}^{\left\lceil C^{\prime}|W|\right\rceil} \leqslant\left(\frac{\mathrm{e}|U|}{C^{\prime}} p_{e}\right)^{C^{\prime}|W|} .
$$

Notamos que $p_{e}|U| \leqslant\left(p_{e} n\right)^{\ell-2} / n \leqslant A^{\ell-2} n^{-1 /(\ell-1)}$. De $|U| \leqslant|W|<\lambda n$, para todo $\lambda>0$, concluímos que $\left(\begin{array}{c}n \\ |U|\end{array}\right) \leqslant\left(\begin{array}{c}n \\ |W|\end{array}\right)$ e, portanto, $\left(\begin{array}{c}n \\ |U|\end{array}\right)\left(\begin{array}{c}n \\ |W|\end{array}\right) \leqslant\left(\begin{array}{c}n \\ |W|\end{array}\right)^{2}$. Essas desigualdades implicam que o número esperado de subconjuntos $U$ e $W$, com cardinalidades $u$ e $w$, respectivamente, para os quais

$$
u \leqslant w \leqslant\left(p_{e} n\right) u \leqslant\left(p_{e} n\right)^{\ell-2}
$$


vale, e tal que $e_{\Gamma}(U, W)>C w$, é menor que

$$
\begin{aligned}
\sum_{w=1}^{\lfloor\lambda\rfloor\rfloor} \sum_{u=1}^{w}\left(\begin{array}{l}
n \\
u
\end{array}\right)\left(\begin{array}{l}
n \\
w
\end{array}\right)\left(\frac{\mathrm{e}}{C^{\prime}} \cdot p_{e} u\right)^{C^{\prime} w} & \\
& \leqslant \sum_{1 \leqslant w<\lambda n} w\left(\begin{array}{l}
n \\
w
\end{array}\right)^{2}\left(\frac{\mathrm{e}}{C^{\prime}} A^{\ell-2} n^{-1 /(\ell-1)}\right)^{C^{\prime} w} \\
& \leqslant \sum_{1 \leqslant w<\lambda n} w\left(\frac{\mathrm{e} n}{w}\right)^{2 w}\left(\frac{\mathrm{e}}{C^{\prime}} A^{\ell-2} n^{-1 /(\ell-1)}\right)^{C^{\prime} w} \\
& \left.=\sum_{1 \leqslant w<\lambda n} \frac{w}{w^{2 w}}\left(\frac{\mathrm{e}^{1+2 / C^{\prime}}}{C^{\prime}} A^{\ell-2} n^{\left(2 / C^{\prime}\right)-(1 /(\ell-1))}\right)\right)^{C^{\prime} w} \\
& \leqslant \sum_{1 \leqslant w<\lambda n}\left(\frac{\mathrm{e}^{1+2 / C^{\prime}}}{C^{\prime}} A^{\ell-2} n^{-2 / C^{\prime}}\right)^{C^{\prime} w} \\
& =\sum_{1 \leqslant w<\lambda n}(o(1))^{C^{\prime} w} \\
= & o(1),
\end{aligned}
$$

Assim, pelo mesmo argumento usado em (28), a probabilidade de existir algum par induzindo muitas arestas tende a 0 quando $n \rightarrow \infty$.

2.2.2. $\mathrm{V}^{(m)}$-subgrafos de $\Gamma$. Tomamos as constantes

$$
C=4(\ell-1) e(H) \text { e } D=\mathrm{e} 2 e(H)
$$

fixamos uma constante $\rho_{0}>0$ e vamos supor que

$$
\alpha \leqslant \frac{\rho_{0} \ell}{\mathrm{e}^{2}(\ell+2)} .
$$

Fixado $k_{0} \in \mathbb{N}$, sejam $\varepsilon, B_{0}$ e $m_{0}$ as constantes que o Lema 19, página 54 , garante existirem, para as constantes dadas acima, e sejam $\eta$ e $K_{0}$ as constantes dadas pelo Lema 9, página 43.

Seja $\mathrm{V}^{(m)}=\left(V_{i}\right)_{i=1}^{\ell}$ um vetor qualquer de subconjuntos dois-a-dois disjuntos de $[n]$, onde $n / 2 K_{0} \leqslant m \leqslant n / k_{0}$. Lembramos que $p_{e}=A n^{-1+1 /(\ell-1)}$.

ProposiçÃo 36. Suponha $A \geqslant 2 B_{0} K_{0}$. Quase certamente $\Gamma$ satisfaz a seguinte propriedade. Se $\mathbf{V}^{(m)}$ e $\Gamma^{(e)} \subset \Gamma$ são tais que os pares $\left(V_{i}, V_{i+1}\right)$ são $\left(\varepsilon, \Gamma^{(e)} ; p_{e}\right)$-regulares de densidade pelo menos $\rho_{0}$, para todo $i \in[\ell]$, então $\Gamma^{(e)}\left[\mathrm{V}^{(m)}\right]$ contém $C^{\ell}$. 
DemonstraÇÃo. Escolha $B$ tal que $p_{e}=B m^{-1+1 /(\ell-1)}$. Suponha que $\mathrm{V}^{(m)}$ e $\Gamma^{(e)}$ são tais que os pares $\left(V_{i}, V_{i+1}\right)$ são $\left(\varepsilon, \Gamma^{(e)}, p_{e}\right)$-regulares de densidade pelo menos $\rho_{0}$, para todo $i \in[\ell]$.

Pelas escolhas de $B, C$ e $D$, e pelas Proposições 34 e 35 , podemos supor que $\Gamma^{(e)}\left[\mathrm{V}^{(m)}\right]$ contém um subgrafo $\ell$-partido que é um $\left(\varepsilon, \rho_{0}, B, C, D ; \mathrm{V}^{(m)}, M\right)$-grafo, onde $M$ é pelo me$\operatorname{nos} \rho_{0} p_{e} m^{2} \ell$.

Agora, notamos que

$$
B=p_{e} m^{1-1 /(\ell-1)} \geqslant 2 B_{0} K_{0}(m / n)^{1-1 /(\ell-1)}>B_{0}\left(2 K_{0} m / n\right)^{1-1 /(\ell-1)} \geqslant B_{0}
$$

onde, a última desigualdade vem do fato de $m 2 K_{0} \geqslant n$, e notamos também que $m \geqslant m_{0}$ para $n$ suficientemente grande. Logo, podemos aplicar o Lema 19.

Fixado $\mathrm{V}^{(m)}$, o valor esperado para o número de $\left(\varepsilon, \rho_{0}, B, C, D ; \mathrm{V}^{(m)}, M\right)$-subgrafos de $\Gamma^{(e)}$ que não contêm circuitos de comprimento $\ell$ é, pela equação (16) da página 54 , no máximo

$$
\alpha^{M}\left(\begin{array}{c}
(\ell+2) m^{2} \\
M
\end{array}\right) p_{e}{ }^{M} \leqslant\left(\frac{\alpha \mathrm{e}(\ell+2) m^{2}}{M} p_{e}\right)^{M} \leqslant\left(\frac{\mathrm{e} \alpha(\ell+2)}{\ell \rho_{0}}\right)^{M}=\left(\frac{1}{\mathrm{e}}\right)^{M} .
$$

Para $m$ e $M$ fixos, somando sobre todas as escolhas possíveis para $\mathbf{V}^{(m)}$ o número esperado de $\left(\varepsilon, \rho_{0}, B, C, D ; \mathbf{V}^{(m)}, M\right)$-subgrafos de $\Gamma^{(e)}$ sem circuitos de comprimento $\ell$ é no máximo

$$
(n)_{\ell m}\left(\frac{1}{\mathrm{e}}\right)^{M} \leqslant n^{\ell m} \exp \left\{-\rho_{0} p_{e} m^{2} \ell\right\} \leqslant \exp \left\{(\log n) \dot{m} \ell-\rho_{0} p_{e} m^{2} \ell\right\}=o\left(n^{-3}\right) .
$$

Finalmente, somando sobre todas as escolhas possíveis para $m \in[n]$ e $M \in\left[\left(\begin{array}{c}n \\ 2\end{array}\right)\right]$, temos um fator adicional de no máximo $n^{3}$. Portanto, a proposição segue da desigualdade de Markov, de modo análogo à (28).

Escrevemos $\mathbf{W}^{(m)}$ para uma $h$-upla $\left(W_{i}\right)_{i=1}^{h}$ de subconjuntos dois-a-dois disjuntos de $[n]$, cada um de cardinalidade $m$.

Proposição 37. Suponha que $A \geqslant(h+1) / \mathrm{e}^{h}$ e que $n / \log n \leqslant m \leqslant n / h$. Para todo $\mathrm{W}^{(m)}$ o subgrafo h-partido de $\Gamma$ induzido por $\mathrm{W}^{(m)}$ quase certamente contém pelo menos menos $(1 / 4) e(H) m^{h} p_{H}$ arestas. 
Demonstração. Fixado uma $h$-upla de subconjuntos dois-a-dois disjuntos $\mathbf{W}=\mathbf{W}^{(m)}$, definimos a variável aleatória $X_{\mathrm{W}}$ como número de hiperarestas de $\mathcal{H}_{n, p_{H}}$ formadas por um vértice de cada $W_{i}$, ou seja, o número de hiperarestas $E$ tais que $\left|E \cap W_{i}\right|=1$, para todo $i \in[h]$. O número esperado de tais hiperarestas é $\mathbb{E} X_{\mathrm{W}}=m^{h} p_{H}$.

Pela desigualdade de Chernoff

$$
\begin{aligned}
\mathbb{P}\left\{X_{\mathrm{W}} \leqslant \frac{1}{2} \mathbb{E} X_{\mathrm{W}}\right\} & \leqslant \exp \left\{-\frac{\mathbb{E} X_{\mathrm{W}}}{8}\right\} \\
& \leqslant \exp \left\{-\frac{A}{8} m^{h} n^{-h+1+1 /(\ell-1)}\right\} \\
& \leqslant \exp \left\{-\frac{A}{8}\left(\frac{n}{\log n}\right)^{h} n^{-h+1+1 /(\ell-1)}\right\} \\
& \leqslant \exp \left\{-\frac{A}{8} \frac{n^{1+1 /(\ell-1)}}{\log ^{h}(n)}\right\}
\end{aligned}
$$

e como há no máximo $2^{h n}$ escolhas para $\mathbf{W}$, a probabilidade de existir $\mathbf{W}$ com $X_{\mathbf{W}} \leqslant$ $(1 / 2) \mathbb{E} X_{\mathrm{W}}$, onde $U=\bigcup_{j=1}^{h} V_{j}$, é menor que

$$
\exp \left\{\left(h-\frac{n^{1 /(\ell-1)}}{\log ^{h}(n)}\right) n\right\}=o(1) .
$$

Ou seja, pela desigualdade de Markov, quase certamente vale $X_{\mathrm{W}}>(1 / 2) \mathbb{E} X_{\mathrm{W}}$.

O número de hiperarestas removidas de $\mathcal{H}_{n, p_{H}}$ é $O(n)$. Logo, o número de hiperarestas induzidas por $\mathrm{W}$ em $\mathcal{G}$ é quase certamente maior que $(1 / 2) \mathbb{E} X_{\mathrm{W}}-O(n) \geqslant(1 / 4) \mathbb{E} X_{\mathrm{W}}$. Como as hiperarestas formam cópias aresta-disjuntas de $H$ em $\Gamma$, temos mais que $(1 / 4) e(H) \mathbb{E} X_{\mathrm{W}}$ arestas induzidas por $\mathrm{W}$ em $\Gamma$.

2.2.3. Subgrafos pequenos de $\Gamma$. Dado um vértice $v \in V(\mathcal{G})$ vamos chamar de hipergrau de $v$ em $\mathcal{G}$, denotado por $d_{\mathcal{G}}(v)$, o número de hiperarestas de $\mathcal{G}$ que contém $v$ como vértice. Denotamos por $\mathcal{G}[U]$ o sub-hipergrafo de $\mathcal{G}$ induzido pelos vértices em $U \subseteq V(\mathcal{G})$. Para o hipergrafo $\mathcal{G}$ obtido a partir de $\mathcal{H}_{n, p_{H}}$ conforme descrevemos acima, vamos provar que todo subconjunto $U \subseteq[n]$ com cardinalidade no máximo $t$, para algum $t>0$, contém um vértice $v$ com hipergrau $d_{\mathcal{G}[U]}(v) \leqslant 1$. 
Proposição 38. Dado um inteiro $t \geqslant 1$, quase certamente todo $U \subseteq[n]$ de cardinalidade no máximo $t$ contém um vértice $v$ com hipergrau $d_{\mathcal{G}[U]}(v) \leqslant d_{\mathcal{H}_{n, p_{H}}[U]}(v) \leqslant 1$.

Demonstração. Fixamos $U \subseteq[n]$ de cardinalidade $u$. Se esses $u$ vértices induzem $m$ hiperarestas e se todo vértice tem grau pelo menos 2 , então nós temos que $m \geqslant 2 u / h$. Agora,

$$
\mathbb{P}\left\{m \geqslant \frac{2 u}{h}\right\} \leqslant\left(\begin{array}{c}
\left(\begin{array}{c}
u \\
h
\end{array}\right) \\
\lceil 2 u / h\rceil
\end{array}\right) p_{H}^{\lceil 2 u / h\rceil}=O\left(n^{-2 u+(2 u / h)(1+1 /(\ell-1))}\right) .
$$

Portanto, a probabilidade de existir um subconjunto $U \subseteq[n]$ com $u$ vértices e pelo menos $2 u / h$ hiperarestas é no máximo

$$
\left(\begin{array}{l}
n \\
u
\end{array}\right) O\left(n^{-2 u+(2 u / h)(1+1 /(\ell-1))}\right)=O\left(n^{-u+(2 u / h)(1+1 /(\ell-1))}\right)=o(1)
$$

pois $h \geqslant 3$ e $\ell \geqslant 4$.

Assim, quase certamente não existe tal conjunto $U$ e o resultado segue.

\section{A demonstração do Teorema 33}

Dados inteiros $\ell \geqslant 4$ e $t \geqslant 3$ e um grafo $H \in \mathcal{C}(\ell)$, sejam

$$
k_{0}=k_{0}\left(K^{2}, C^{\ell}, K^{h}\right) \text { e } c=c\left(K^{2}, C^{\ell}, K^{h}\right)
$$

as constantes dadas pelo Lema 5 na página 28. Definimos

$$
\rho_{0}=\frac{c}{2^{h+2}}, \alpha=\frac{\rho_{0} \ell}{\mathrm{e}^{2}(\ell+2)}, C=4(\ell-1) e(H) \text { e } D=\mathrm{e} 2 e(H) .
$$

Sejam

$$
\varepsilon=\varepsilon\left(\ell, \alpha, \rho_{0}, C, D\right) B_{0}=B_{0}\left(\ell, \alpha, \rho_{0}, C, D\right) \text { e } m_{0}=m_{0}\left(\ell, \alpha, \rho_{0}, C, D\right)
$$

as constantes que o Lema 19 garante existirem e

$$
\eta=\eta\left(\varepsilon, D, k_{0}\right) \text { e } K_{0}=K_{0}\left(\varepsilon, D, k_{0}\right)
$$

as constantes dadas pelo Lema 9. Assumimos que $\varepsilon<\min \{1 / 2,2 c\}$ e atribuímos

$$
A=\max \left\{2 B_{0} K_{0},(h+1) \mathrm{e}^{-h}\right\}
$$

Para esses valores de constantes dados acima, considere a família $\mathcal{F}=\mathcal{F}(H)$ dos grafos $\Gamma$ sobre $[n]$ que podem ser escritos como uma união de $M$ cópias de $H$, digamos $H_{1}, \cdots, H_{M}$, 
onde $M \asymp n^{2} p_{e}$, tais que quaisquer duas cópias compartilham no máximo um vértice. Para cada $\Gamma \in \mathcal{F}$ nós fixamos uma tal família $H_{1}, \ldots, H_{M}$ de cópias de $H$ e as chamamos de cópias não-espontâneas de $H$ em $\Gamma$; qualquer outra cópia de $H$ em $\Gamma$ é chamada de espontânea. Além disso, os grafos de $\mathcal{F}$ satisfazem as seguintes propriedades:

(P1) o hipergrafo sobre $[n]$ dado pelas hiperarestas $\left\{V\left(H_{i}\right): i \in[M]\right\}$ tem cintura $\ell$;

(P2) $\Gamma$ é $\left(\eta, D, p_{e}\right)$-esparso e $\left(p_{e}, C, \ell\right)$-localmente-esparso;

(P3) para todo $\mathrm{V}^{(m)}=\left(V_{1}, \ldots, V_{\ell}\right)$, onde $n /\left(2 K_{0}\right) \leqslant m \leqslant n / k_{0}$, se $\Gamma^{(e)} \subseteq \Gamma\left[\mathbf{V}^{(m)}\right]$ é tal que os pares $\left(V_{i}, V_{i+1}\right)$ são $\left(\varepsilon, \Gamma^{(e)}, p_{e}\right)$-regulares de $p_{e}$-densidade pelo menos $\rho_{0}$, para todo $1 \leqslant i \leqslant \ell$, então $\Gamma^{(e)}$ contém $C^{\ell}$;

(P4) todo $\mathbf{W}^{(m)}=\left(W_{1}, \ldots, W_{h}\right)$, onde $m \geqslant n / \log n$ induz pelo menos $(1 / 4) e(H) m^{h} p_{H}$ arestas de $\Gamma$ que podem ser estendidas para cópias não-espontâneas $H_{i}$ de $H$ contidas em $\bigcup_{1 \leqslant j \leqslant \ell} W_{j}$

(P5) todo subconjunto $U \subseteq[n]$ com $t$ vértices contém um vértice $v_{U}$ que pertence a no máximo uma cópia não-espontânea $H_{i}$ de $H, \operatorname{com} V\left(H_{i}\right) \subseteq U$.

Pelas Proposições 34 - 38, na Seção 2.2, a família $\mathcal{F}$ não é vazia. O fato do grafo $H$ pertencer ao conjunto $\mathcal{C}(\ell)$ e $\Gamma$ satisfazer (P1) implicam a seguinte propriedade cuja prova fica adiada para a próxima seção.

(P6) $\Gamma$ não contém cópias espontâneas de $H$.

Fixamos um grafo $\Gamma \in \mathcal{F}$ e, agora, passamos a mostrar que $\Gamma \rightarrow\left(C^{\ell}, H\right)$ e que $\Gamma \nrightarrow_{t}$ $\left(C^{\ell}, H\right)$.

3.1. Demonstração de $\Gamma \rightarrow\left(C^{\ell}, H\right)$. Sejam $H_{1}, \ldots, H_{M}$ as cópias não-espontâneas de $H$ em $\Gamma$ e seja $\Gamma^{(e)}$ um subgrafo gerador de $\Gamma$ obtido colorindo de vermelho uma aresta de cada cópia $H_{i} \subseteq \Gamma$ de $H$. Vamos mostrar que essas arestas geram uma cópia vermelha de um $C^{\ell}$.

Por (P2), sabemos que o grafo $\Gamma^{(e)}$ é $\left(\eta, D, p_{e}\right)$-esparso. Aplicamos a versão esparsa do Lema de Szemerédi a esse grafo e temos uma partição $\varepsilon$-regular $\Pi=\left(V_{0}, V_{1}, \ldots, V_{k}\right)$. Vamos escrever $m$ para a cardinalidade comum das partes não-excepcionais de П. Claramente,

$$
\frac{n}{2 K_{0}} \leqslant(1-\varepsilon) \frac{n}{k} \leqslant m \leqslant \frac{n}{k} \leqslant \frac{n}{k_{0}} .
$$


Seja $K^{k}$ o grafo completo com conjunto de vértices $V\left(K^{k}\right)=\left\{V_{1}, V_{2}, \ldots, V_{k}\right\}$ e considere a seguinte 3-coloração das arestas do $K^{k}$ :

- a cor de $V_{i} V_{j}$ é 1 se o par $\left(V_{i}, V_{j}\right)$ não é $\varepsilon$-regular,

- a cor de $V_{i} V_{j}$ é 2 se é regular de densidade $\geqslant \rho_{0}$, e

- a cor de $V_{i} V_{j}$ é 3 se é regular de densidade $<\rho_{0}$.

Por $k \geqslant k_{0}$, pela escolha de $k_{0}$ e pelo Lema 5 da página 28 nós temos que o $K^{k}$ deve conter

(i) pelo menos $c k^{2}$ cópias do $K^{2}$ da cor 1 , ou

(ii) pelo menos $c k^{\ell}$ cópias do $C^{\ell}$ da cor 2 , ou

(iii) pelo menos $c k^{h}$ cópias do $K^{h}$ da cor 3 .

O caso (i) contraria o fato de $<\varepsilon\left(\begin{array}{c}k \\ 2\end{array}\right) \leqslant 2 c\left(\begin{array}{c}k \\ 2\end{array}\right)<c k^{2}$ pares serem irregulares. No caso (ii), havendo uma cópia monocromática de um $C^{\ell} \subset K^{k}$, digamos $C^{\ell}=V_{i_{1}}, \ldots, V_{i_{\ell}}$, temos um vetor $\mathbf{V}^{(m)}=\left(V_{i_{j}}\right)_{j=1}^{\ell}$ que está de acordo com as hipóteses de (P3), ou seja, temos um circuito $C^{\ell}$ da cor vermelha. Vamos supor o caso (iii) e derivar uma contradição, terminando assim a prova de que $\Gamma \rightarrow\left(C^{\ell}, H\right)$.

$\mathrm{O}$ número de arestas de $\Gamma^{(e)}$ no pares $\left(V_{i}, V_{j}\right)$ que correspondem às arestas de cor 3 do $K^{k}$ é

$$
\begin{gathered}
\sum_{\substack{V_{i} V_{k} \in E\left(K^{k}\right) \\
\operatorname{cor}\left(V_{i} V_{j}\right)=3}} e_{\Gamma(e)}\left(V_{i}, V_{j}\right) \leqslant\left(\begin{array}{l}
k \\
2
\end{array}\right) d_{p_{e}}\left(V_{i}, V_{j}\right) p_{e} m^{2}<\left(\begin{array}{c}
k \\
2
\end{array}\right) \rho_{0} p_{e} m^{2} \leqslant\left(\begin{array}{l}
k \\
2
\end{array}\right) \rho_{0} p_{e}\left(\frac{n}{k}\right)^{2}< \\
\left(1-\frac{1}{k}\right) \frac{1}{2} A \rho_{0} n^{1+1 /(\ell-1)}<\rho_{0} A n^{1+1 /(\ell-1)}=\frac{c}{2^{h+3}} A n^{1+1 /(\ell-1)}
\end{gathered}
$$

Por outro lado, por (iii) temos pelo menos $c k^{h}$ cópias do $K^{h} \subset K^{k}$. Fixe uma dessas cópias e denote por $J$ o subgrafo $h$-partido de $\Gamma$ que está naturalmente associado, pela definição do grafo $K^{k}$, a esse $K^{h}$. Cada classe de $J$ tem $m \geqslant n / 2 k \geqslant n / \log n$ vértices. Por (P4), $J$ induz $\geqslant(1 / 4) e(H) m^{h} p_{H}$ arestas, portanto, $J$ contém pelo menos $(1 / 4) m^{h} p_{H}$ cópias de $H$. Assim, temos pelo menos $(1 / 4) m^{h} p_{H}$ arestas de $\Gamma^{(e)} \mathrm{em} J$.

Quaisquer dois desses $K^{h}$ 's de cor $3 \mathrm{em} K^{k}$ determinam conjuntos disjuntos de arestas em $\Gamma^{(e)}$, pois cada aresta de $\Gamma^{(e)}$ está contida numa única cópia não-espontânea de $H \subseteq \Gamma$. 
Portanto, o número de arestas induzidas por essas $c k^{h}$ cópias do $K^{h}$ é pelo menos

$$
c k^{h} \frac{1}{4} m^{h} p_{H} \geqslant c k^{h} \frac{1}{4}\left(\frac{n}{2 k}\right)^{h} A n^{-h+1+1 /(\ell-1)} \geqslant \frac{c}{2^{h+2}} A n^{1+1 /(\ell-1)} .
$$

Como as duas desigualdades acima são contraditórias, concluímos que deve haver um circuito $C^{\ell}$ com todas as arestas da cor vermelha.

3.2. A prova de $\Gamma \dashv_{t}\left(C^{\ell}, H\right)$. Agora, vamos provar por indução em $|U|$ que, para todo $U \subseteq V(\Gamma)$ com no máximo $t$ vértices, o subgrafo $\Gamma[U]$ não é ramsey com respeito ao par $\left(C^{\ell}, H\right)$. Fixamos $U \subset[n]$. Se $|U|<\max \{\ell, h\}$, então $\Gamma[U] \not \rightarrow\left(C^{\ell}, H\right)$.

Seja $U$ um subconjunto de cardinalidade $\max \{\ell, h\} \leqslant|U| \leqslant t$ e vamos assumir que $\Gamma\left[U^{\prime}\right] \nrightarrow\left(C^{\ell}, H\right)$, para todo $U^{\prime} \subset U$.

Pela propriedade (P5), o conjunto $U$ contém um vértice $v_{U}$ que pertence a no máximo uma cópia não-espontânea de $H$ totalmente contida em $U$. Vamos denotar por $H_{i_{0}}$ essa cópia de $H$, se ela existe. Pela hipótese de indução, o subgrafo $\Gamma\left[U \backslash\left\{v_{U}\right\}\right]$ admite uma vermelho-azul aresta-coloração sem $C^{\ell}$ monocromático da cor vermelha e sem $H$ monocromático da cor azul. Fixamos uma tal coloração.

Agora, vamos colorir as arestas incidentes em $v_{U}$. Podemos pintar exatamente uma das arestas que incidem em $v_{U}$, digamos $e$, de vermelho que não criamos $C^{\ell}$ na cor vermelha. Se for o caso, escolhemos $e \in E\left(H_{i_{0}}\right)$, assim eliminamos a possibilidade de aparecer uma cópia não-espontânea de $H$ com todas as arestas da cor azul, caso contrário, escolhemos $e$ arbitrariamente. As outras arestas que incidem em $v_{U}$ nós pintamos de azul. Se essa coloração gera uma cópia $H^{\prime}$ azul de $H$ em $\Gamma[U] \subset \Gamma$, então $H^{\prime}$ é uma cópia espontânea de $H$. Mas, por (P6), tal cópia não existe. Portanto, temos uma aresta-coloração de $\Gamma[U]$ que mostra que $\Gamma[U] \nrightarrow \rightarrow\left(C^{\ell}, H\right)$.

\section{Sobre a Propriedade (P6)}

Considere $\Gamma \in \mathcal{F}=\mathcal{F}(H)$ e sejam $H_{1}, \ldots, H_{M}$ as suas cópias não-espontâneas de $H$. Agora, vamos provar que o fato do grafo $H$ pertencer a $\mathcal{C}(\ell)$ e $\Gamma$ satisfazer (P1) implicam que $\Gamma$ não contém cópias espontâneas de $H$. 
A prova é por contradição. Suponha que $\widetilde{H}$ é uma cópia espontânea de $H$ em $\Gamma$. Então, existem duas arestas em $\widetilde{H}$ pertencentes a diferentes cópias não-espontâneas de $H$ em $\Gamma$, digamos $e, f \in E(\tilde{H})$ tais que existem únicos $i(e), i(f) \in[M] \operatorname{com} e \in E\left(H_{i(e)}\right)$ e $f \in E\left(H_{i(f)}\right)$. Como $\widetilde{H}$ é 2-conexo, existe um circuito $C \subset \widetilde{H}$ tal que $e, f \in E(C)$. Esse circuito $C$ não pode estar inteiramente contido numa das cópias não-espontânea $H_{i}$ de $H$, portanto $C$ deve ter comprimento pelo menos $\ell$ pela propriedade (P1).

Vamos supor que o circuito $C$ é o de menor comprimento dentre todos os circuitos de $\widetilde{H}$ que têm pelo menos duas arestas em diferentes cópias não-espontâneas de $H$. Pela definição da classe $\mathcal{C}(\ell)$, o circuito $C$ deve ter uma corda $c$. Pela minimalidade de $C$, temos que $c \notin E\left(H_{i(e)}\right)$, para todo $e \in E(C)$. Logo, deve existir $i(c) \in[M]$ tal que a cópia nãoespontânea $H_{i(c)}$ contém a aresta $c$ e é diferente de todos os $H_{i(e)}$ para $e \in E(C)$. Mas isso implica num circuito $C^{\prime} \subset \widetilde{H}$ tal que $V\left(C^{\prime}\right) \subset V(C)$ e $C^{\prime}$ tem pelo menos duas arestas em diferentes cópias não-espontâneas de $H$, a aresta $c$ e, por exemplo, uma das arestas adjacentes a $c$ em $C^{\prime}$. Esse fato contradiz a minimalidade de $C$ e completa a nossa demonstração.

\section{Considerações finais}

Lembramos o leitor que na Introdução deste capítulo dissemos que praticamente todos pares ramsey-finitos formados por duas florestas estão caracterizados por um teorema de Faudree (1991) e que Nešetřil e Rödl (1978) provaram que o par $(G, H)$ é ramsey-infinito se $G$ e $H$ são 3-conexos. Esses resultados deixam um "gap" quando $G$ e $H$ têm conexidade 2 ou menos.

Como foi dito, mesmo o caso $\left(C^{\ell}, H\right)$ quando $H$ é 2-conexo é desconhecido e, infelizmente, o nosso resultado tem a hipótese de circuitos longos não serem induzidos. Essa hipótese é essencial para a prova de $\Gamma \nrightarrow_{t}\left(C^{\ell}, H\right)$. Portanto, é interessante tentar remover essa hipótese, ou mesmo substituí-la por uma mais fraca e que garanta (P6).

Finalmente, cabe mencionar que neste capítulo provamos um resultado mais forte do que está enunciado. Nós provamos que uma vermelho-azul aresta-coloração de $\Gamma$ resulta, ou numa cópia induzida vermelha de $C^{\ell}$, ou uma cópia induzida azul de $H$, ou seja, o par $\left(C^{\ell}, H\right)$ é o 
que se chama de fortemente-ramsey-infinito. Esse conceito foi investigado, por exemplo, por Burr (1986), por Burr et al. (1985), e por Nešetřil e Rödl (1978). 


\section{CAPíTULO 4}

\section{O NÚMERO DE RAMSEY-ARESTA DE SUBDIVISÕES DE GRAFOS}

Dados os grafos $H_{1}, \ldots, H_{q}$, definimos $\hat{r}\left(H_{1}, \ldots, H_{q}\right)$ como o menor número de arestas $m$ tal que existe um grafo $\Gamma$ com $m$ arestas e ramsey para a $q$-upla de grafos $\left(H_{1}, \ldots, H_{q}\right)$, isto é, em símbolos

$$
\hat{r}\left(H_{1}, \ldots, H_{q}\right)=\min \left\{e(\Gamma): \Gamma \rightarrow\left(H_{1}, \ldots, H_{q}\right)\right\}
$$

Quando $H_{1}=\cdots=H_{q}=H$, usamos a notação abreviada $\hat{r}(H ; q)$ para indicar o número $\hat{r}\left(H_{1}, \ldots, H_{q}\right)$. Em particular, escrevemos $\hat{r}(H)$ para $\hat{r}(H, H)$.

Dado um grafo $H$, denotamos por $H^{(s)}$ o grafo obtido a partir de $H$ subdividindo $s-1$ vezes cada aresta de $H$.

Neste capítulo nós mostramos que, dados $H$ e o número de cores $q \geqslant 2$, existem constantes positivas $C_{1}$ e $C_{2}$ e grafos $G^{n}$ com $O(n)$ arestas tais que $G^{n} \rightarrow\left(H^{(s)} ; q\right)$, para todo $n$ suficientemente grande, onde $C_{1} \lg n \leqslant s \leqslant C_{2} n$.

\section{Introdução}

O problema de estudar o número de ramsey para arestas, que passamos a chamar de número de ramsey-aresta, foi proposto por Erdős et al. (1978) quando foi provado, dentre outros resultados, que $\hat{r}\left(K^{1, n}, K^{1, m}\right)=m+n+1$. A partir desse trabalho seminal, vários autores contribuíram para o assunto. Vamos descrever algumas dessas contribuições. Erdős et al. (1978) perguntaram se o limite

$$
\lim _{n \rightarrow \infty} \frac{\hat{r}\left(P^{n}\right)}{n}
$$

existe e, se a resposta for sim, determinar esse limite. Beck (1983) mostrou que $\hat{r}\left(P^{n}\right)<900 n$. $\mathrm{Na}$ verdade, esse resultado é corolário doutro que afirma a existência de grafos $G^{n} \operatorname{com} \leqslant 900 n$ 
arestas tais que todo subgrafo $H \subseteq G^{n} \operatorname{com} e(H) \geqslant(1 / 2) e\left(G^{n}\right)$ contém $P^{n}$, ou seja, um resultado do tipo densidade.

No caso do número de ramsey-aresta para árvores, começamos com a seguinte observação: seja $T$ a árvore obtida da estrela $K^{1,[n / 2]}$ subdividindo cada aresta uma vez. Pela equação (2), página 23, devemos ter $\hat{r}(T)>n^{2} / 8$ pois essa árvore tem grau máximo $\Delta(T)=\lceil n / 2\rceil$ e $\lambda(T)=\lceil n / 2\rceil$. Por outro lado, seja $G$ um grafo com $t$ vértices e $n t$ arestas; o grafo $G$ contém qualquer árvore com $n$ arestas (veja página 28), logo o número de ramsey de $\left(T_{n}, T_{n}\right)$ é no máximo $4 n+1$, para qualquer árvore $T_{n}$ com $n$ arestas. Concluímos que $\hat{r}\left(T_{n}\right)<17 n^{2}$.

Beck (1983) conjecturou que toda árvore com grau limitado tem número de ramsey-aresta linear. Vamos denotar por $T_{n, \Delta}$ uma árvore $\operatorname{com} n$ arestas e grau máximo $\Delta$. A conjectura de Beck (1983) é que $\hat{r}\left(T_{n, \Delta}\right)<c \Delta n$, para alguma constante universal $c$; ele conseguiu demonstrar que $\hat{r}\left(T_{n, \Delta}\right)<\Delta n(\log n)^{12}$, para todo $n$ suficientemente grande.

O problema de saber se o número de ramsey-aresta para árvores com grau limitado é linear foi resolvido por Friedman e Pippenger (1987) que provaram $\hat{r}\left(T_{n, \Delta}\right)=O\left(\Delta^{4} n\right)$. Ke (1993) melhorou esse resultado mostrando que $\hat{r}\left(T_{n, \Delta}\right)=O\left(\Delta^{2} n\right)$. Haxell e Kohayakawa (1995) mostraram que $\hat{r}\left(T_{n, \Delta}\right) \leqslant c_{1} \Delta n$, verificando a conjectura de Beck (1983).

Agora, chamamos a atenção para o seguinte fenômeno que ocorre com estrelas: o número ramsey-aresta $\hat{r}\left(K^{1, n}\right)$ é $2 n-1$, enquanto que $\Delta n=(n-1) n$, logo o limitante $O(\Delta n)$ é quadrático, longe do valor correto. Na tentativa de acertar a ordem de grandeza de $\hat{r}(T)$ para árvores, Beck (1990) conjecturou o seguinte: se a árvore $T$ tem bipartição $V(T)=A \cup B$, com $|A|=n_{0}$ e os vértices de $A$ têm grau no máximo $\Delta_{0}$, e com $|B|=n_{1}$ e os vértices de $B$ têm grau no máximo $\Delta_{1}$, então $\hat{r}(T) \leqslant c \beta(T)$, para $\beta(T)=n_{0} \Delta_{0}+n_{1} \Delta_{1}$ e $c$ constante. Nesse mesmo trabalho, o autor mostrou que $\beta(T) / 4 \leqslant \hat{r}(T) \leqslant c \beta(T)(\log v(T))^{12}$.

Nessa direção, Haxell e Kohayakawa (1995) mostraram que $\hat{r}(T) \leqslant c_{2} \beta(T) \log \Delta(T)$, onde $c_{1}$ e $c_{2}$ são constantes.

Beck (1990) ainda perguntou se grafos $G_{n, \Delta}$ de ordem $n$ e grau máximo $\Delta$ têm número de ramsey-aresta linear, ou seja, $\hat{r}\left(G_{n, \Delta}\right) \leqslant c n$, para alguma constante $c=c(\Delta)$. Haxell et al. (1995a) mostraram que esse é o caso para circuitos, ou seja, $\hat{r}\left(C^{\ell}\right)=O(\ell)$. Rödl e Szemerédi (2000) mostraram um contra-exemplo para essa conjectura: existem constantes positivas c e 
$\alpha$ e um grafo $G^{n}$ de grau máximo 3 tal que $\hat{r}(G) \geqslant c n(\log n)^{\alpha}$; esses autores conjecturaram, no mesmo trabalho, o seguinte.

Conjectura 39 (Rödl e Szemerédi, 2000). Para todo $\Delta \in \mathbb{N}$ existe $\varepsilon>0$ tal que

$$
n^{1+\varepsilon} \leqslant \hat{r}(n, \Delta) \leqslant n^{2-\varepsilon},
$$

para $\hat{r}(n, \Delta)=\max \hat{r}(G)$, onde o máximo é tomado sobre todos os grafos $G$ de ordem $n$ e grau máximo $\Delta$.

Kohayakawa e Rödl (2001) afirmam que, juntamente com Szemerédi (veja Kohayakawa et al., 2001), provaram a cota superior da conjectura acima.

1.1. Definições e o resultado principal. Antes de enunciar nosso resultado, vamos rever algumas definições. Fixamos um grafo $H$. Dada uma função $s: E(H) \rightarrow \mathbb{N}$, definimos o grafo $H^{(s)}$ substituindo cada aresta $x y \in E(H)$ por um $x-y$ caminho, digamos $P_{x y}$, de comprimento $s(x y)$ de maneira que $P_{x y} \cap P_{z w}=\emptyset$ se $\{x, y\} \neq\{z, w\}$ e caso contrário, digamos $x=z$, então $P_{x y} \cap P_{z w}=\{x\}$. Chamamos $H^{(s)}$ de uma subdivisão de $H$.

Estamos interessados no caso em que $s$ é uma função constante. Quando dizemos que $H^{(s)}$ é uma subdivisão de $H$ com $a \leqslant s \leqslant b$, para $a$ e $b$ inteiros positivos, queremos dizer que $\operatorname{Im}(s)=k$ e que $a \leqslant k \leqslant b$, ou seja, cada aresta de $H$ é substituída por um caminho de comprimento $k$ entre $a$ e $b$.

Dados $0<p \leqslant 1$ e $A>0$, escrevemos $d=p n$ e dizemos que um grafo $G=G^{n}$ é $(p, A)$ uniforme se para qualquer par de subconjuntos disjuntos $U, W \subseteq V(G) \operatorname{com}|U| \leqslant|W| \leqslant d|U|$ vale que

$$
\left|e_{G}(U, W)-p\right| U|| W|| \leqslant A \sqrt{d|U||W|} .
$$

O número de arestas num grafo $(p, A)$-uniforme $G$ é, somando-se sobre todos os subconjuntos $U$ e $W=U \backslash V \operatorname{com}|U|=u=\lfloor n / 2\rfloor$,

$$
e(G)=\frac{\sum_{U, W} e(U, W)}{2\left(\begin{array}{l}
n-2 \\
u-1
\end{array}\right)} \leqslant \frac{\left(\begin{array}{l}
n \\
u
\end{array}\right) p u(n-u)+A \sqrt{d u(n-u)}}{2\left(\begin{array}{l}
n-2 \\
u-1
\end{array}\right)},
$$


portanto,

$$
e(G) \leqslant p\left(\begin{array}{l}
n \\
2
\end{array}\right)+2 A \sqrt{d\lfloor n / 2\rfloor\lceil n / 2\rceil} \leqslant p\left(\begin{array}{l}
n \\
2
\end{array}\right)+A \sqrt{d}(n+1) .
$$

Da mesma forma, $e(G) \geqslant p\left(\begin{array}{l}n \\ 2\end{array}\right)-A \sqrt{d}(n+1)$, logo

$$
e(G) \geqslant\left(1-\frac{3 A}{\sqrt{d}}\right) p \frac{n^{2}}{2}
$$

O resultado que vamos provar neste capítulo é o seguinte.

TeOrema 40. Dados inteiros $q \geqslant 2$ e $A>0$ e um grafo $H$, existem constantes $C_{0}, C_{1}$ e $C_{2}$ tais que, para todo $n$ suficientemente grande, se $G^{n}$ é um grafo $(p, A)$-uniforme com $d=p n \geqslant C_{0}$, então $G^{n} \rightarrow\left(H^{(s)} ; q\right)$ para todo $C_{1} \lg n \leqslant s \leqslant C_{2} n$.

Portanto, o número de ramsey-aresta para subdivisões "longas" (mais que cn vezes cada aresta, para qualquer $c>0$ constante) de um grafo cujo tamanho não depende de $n$ é linear para todo $n$ suficientemente grande. Assim, respondemos parcialmente a seguinte conjectura.

Conjectura 41 (Pak, 2001). Dado $\Delta \in \mathbb{N}$, seja $H$ um grafo de grau máximo $\Delta$ e considere a subdivisão $H^{(s)}$ com $s>C \log \left|V\left(H^{(s)}\right)\right|$, onde $C>0$ é uma constante. Então $\hat{r}\left(H^{(s)} ; q\right)=O\left(\left|V\left(H^{(s)}\right)\right|\right)$, onde a constante implícita na notação $O(\cdot)$ depende somente de $q$ e de $\Delta$ e não depende do número de vértices de $H^{(s)}$.

Na Seção 5, no final deste capítulo, discutiremos um pouco mais sobre nosso resultado e a conjectura acima.

\section{Resultados auxiliares}

Nesta seção, apresentamos os resultados que serão usados para demonstrar o Teorema 40.

2.1. Grafos expansíveis. Para os próximos dois lemas, seja $B=(U \cup W, E)$ um grafo bipartido e sejam $b$ e $f$ números reais positivos. Dizemos que $B$ é $(b, f)$-expansível quando para todo $X \subseteq U$, ou $X \subseteq W$, se $|X| \leqslant b$ então $|\Gamma(X)| \geqslant f|X|$. Se $B$ não é vazio, então $f>1$ implica que $|U|,|W|>b$. 
Lema 42 (Pósa, 1976). Se $B$ é $(b, 2)$-expansivel, então $B$ contém um caminho com $4 b$ vértices.

Demonstração. A seguinte demonstração é de Haxell (1997). Digamos que $P_{0}=$ $x_{1}, \ldots, x_{k}$ é um caminho de maior comprimento em $B$, supomos, sem perda de generalidade, que $x_{k} \in U$. Observamos que se $x_{1} x_{i} \in E(B)$, então $x_{i-1}, \ldots, x_{1}, x_{i}, \ldots, x_{k}$ também é um caminho de maior comprimento em $B$, que chamamos de uma transformação simples de $P_{0}$.

Uma transformação de $P_{0}$ é um caminho que pode ser obtido a partir de $P_{0}$ por uma seqüência de transformações simples.

Chamamos a atenção para o fato de $x_{k}$ sempre ser um vértice terminal de uma transformação de $P_{0}$. Denotamos por $T$ o subconjunto dos vértices de $P_{0}$ que são terminais de alguma transformação de $P_{0}$, com a exceção de $x_{k}$. Denotamos por $V$ o subconjunto de vértices de $P_{0}$ que são vizinhos em $P_{0}$ de algum vértice de $T$, assim $x_{i} \in V$ se $x_{i-1}$ ou $x_{i+1}$ pertencem a $T$.

Por essas definições, temos que $T \subseteq U$ e $V \subseteq W$ ou vice-versa. Também $|V| \leqslant 2|T|-1$, pois cada vértice terminal contribui com no máximo dois vizinhos, menos o vértice terminal $x_{1}$, que contribui com um vizinho. Obviamente $\Gamma_{B}(T) \subseteq P_{0}$, caso contrário teríamos um caminho maior que $P_{0}$. Agora, vamos verificar que $\Gamma_{B}(T) \subseteq V$.

Tomamos $x_{i} \in \Gamma_{B}(T)$. Seja $P_{z}$ a transformação de $P_{0}$ cujo vértice terminal $z \in T\left(z \neq x_{k}\right)$ é adjacente a $x_{i}$, e tome $x$ o vértice adjacente a $x_{i}$ no $z-x_{i}$ caminho em $P_{z}$. Então $x \in T$, pois $z x_{i} \in B$. Se $x_{i}$ é adjacente a $x$ em $P_{0}$, então $x_{i} \in V$. Portanto, vamos assumir que $x_{i}$ não é adjacente a $x$ em $P_{0}$. Notamos que $x_{i}=x_{1}$ não é possível pois $x_{i} \in \Gamma_{B}(T)$ e $x_{1} \in T$. Agora, para algum vizinho $y$ de $x_{i}$ em $P_{0}$, a aresta $x_{i} y$ não está em $P_{z}$. Chamamos de $P^{\prime}$ a primeira transformação, da seqüência de transformações simples de $P_{0}$ até $P_{z}$, que não contém a aresta $x_{i} y$. O vértice terminal de $P^{\prime}$, que não o vértice $x_{k}$, é, ou o vértice $x_{i}$, ou o vértice $y$, mas $x_{i} \notin T$ desde que $x_{i} \in \Gamma_{B}(T)$, portanto $y \in T$. Logo $x_{i} \in V$.

Agora, $|T| \geqslant b+1$ pois $B$ é $(b, 2)$-expansível e $\left|\Gamma_{B}(T)\right| \leqslant|V| \leqslant 2|T|-1$. Tomamos $T^{\prime} \subseteq T$ de cardinalidade $\left|T^{\prime}\right|=b$ de forma que $\left|\Gamma_{B}\left(T^{\prime}\right)\right| \geqslant 2 b$. Se $T^{\prime} \subseteq U$ então $\Gamma\left(T^{\prime}\right) \subseteq W$ de forma que $\left|P_{0}\right| \geqslant 4 b$. Se $T^{\prime} \subseteq W$ então $\left|P_{0}\right|$ é par e $\left|P_{0} \cap U\right| \geqslant 2 b$ e assim $\left|P_{0}\right| \geqslant 4 b$. 
Definimos a vizinhança $\ell$ vezes iterada de $x \in V(B)=U \cup W$ por

$$
\Gamma^{(\ell)}(x)=\{y \in V(B): \text { existe um } x-y \text { caminho de comprimento } \ell \text { em } B\} .
$$

LemA 43. Se $B$ é $(b, 4)$-expansivel, então para todo vértice $x$ de $B$ e todo $1 \leqslant \ell \leqslant 2 \lg (b)+1$ temos $\left|\Gamma^{(\ell)}(x)\right| \geqslant 2^{\lfloor\ell / 2\rfloor}$.

Demonstração. Vamos definir subconjuntos disjuntos $N_{0}, N_{1}, \ldots, N_{\ell}$ indutivamente da seguinte forma. Primeiro, $N_{0}=\{x\} \subseteq U$ é o nosso vértice de partida. Conforme esses conjuntos vão sendo definidos, teremos $N_{i} \subseteq U$, se $i$ for par, e $N_{i} \subseteq W$ caso contrário.

Suponha estarem definidos $N_{0}, \ldots, N_{t-1}$ disjuntos e tais que $\left|N_{i}\right|=2^{\lfloor i / 2\rfloor}$, para todo $0 \leqslant i \leqslant t-1$.

Observamos que $\left|N_{t-1}\right|=2^{\lfloor(t-1) / 2\rfloor} \leqslant 2^{(\ell-1) / 2} \leqslant b$. Portanto, como estamos assumindo que o grafo é $(b, 4)$-expansível, concluímos que $\left|\Gamma\left(N_{t-1}\right)\right| \geqslant 4\left|N_{t-1}\right| \geqslant 2^{(t / 2)+1}$.

Se $t$ é par, definimos $\mathcal{N}_{t}$ como a união dos $N_{2 j}$, para $0 \leqslant 2 j<t$, cuja cardinalidade é $\sum_{j=0}^{(t / 2)-1} 2^{j}=2^{t / 2}-1$. Logo, podemos escolher $N_{t} \subseteq \Gamma\left(N_{t-1}\right) \backslash \mathcal{N}_{t}$ de cardinalidade $2^{t / 2}$, pois $\left|\Gamma\left(N_{t-1}\right)\right| \geqslant 2^{(t+2) / 2}$ e $\left|\mathcal{N}_{t}\right|=2^{t / 2}-1$.

Se $t$ é ímpar, definimos $\mathcal{N}_{t}$ como a união dos $N_{2 j+1}$, para $0<2 j+1<t$, cuja cardinalidade é $\sum_{j=0}^{((t-1) / 2)-1} 2^{j}=2^{(t-1) / 2}-1$. Logo, podemos escolher $N_{t} \subseteq \Gamma\left(N_{t-1}\right) \backslash \mathcal{N}_{t}$ de cardinalidade $2^{(t-1) / 2}$.

Para finalizar, quando $t=\ell$, temos $N_{\ell} \subseteq \Gamma\left(N_{\ell-1}\right) \subseteq \Gamma\left(\Gamma\left(N_{\ell-2}\right)\right) \subseteq \cdots \subseteq \Gamma^{(\ell)}(x) \mathrm{e}$ $\left|N_{\ell}\right| \geqslant 2^{\lfloor\ell / 2\rfloor}$.

2.2. Caminhos longos em quádruplas regulares. Para os resultados desta seção, são dadas as constantes reais positivas $\rho_{0}$ e $A$. Escolhemos

$$
\varepsilon<\min \left\{\frac{1}{4}, \frac{\rho_{0}}{2\left(5+3 \rho_{0}\right)}\right\} .
$$

Seja $G=G^{n}$ um grafo e consideremos uma quádrupla de subconjuntos dois-a-dois disjuntos de $V(G)$

$$
\mathbf{P}^{(m)}=\left(V_{1}, V_{2}, V_{3}, V_{4}\right)
$$

tal que 
- $\left|V_{j}\right|=m \geqslant \alpha n$ para todo $j \in\{1,2,3,4\}$ e para algum $0<\alpha<1$,

- os pares $\left(V_{i}, V_{j}\right)$ são $(\varepsilon ; p)$-regulares com $p$-densidade $d_{p}\left(V_{i}, V_{j}\right)=d_{i, j} \geqslant \rho_{0}$, para todos $i, j \in\{1,2,3,4\}$.

Definimos

$$
\delta=\frac{\varepsilon}{\rho_{0}}+3 \varepsilon
$$

e escolhemos $d$ tal que

$$
\left(\frac{\alpha \rho_{0} \delta}{A}\right)^{2} d \geqslant 4
$$

Supomos que $G=G^{n}$ é um grafo $(p, A)$-uniforme para $p=d / n$ e $\mathbf{P}^{(m)}$ é uma quádrupla de subconjuntos de $G$ como acima.

Proposição 44. Para todo $i \in\{1,2,3,4\}$, existe $U_{i} \subseteq V_{i}$ de cardinalidade $\left|U_{i}\right| \geqslant(1-$ $3 \varepsilon) m$ tal que para todo $x \in U_{i}$ e para todo $j \in\{1,2,3,4\}$, com $j \neq i$, vale que

$$
\left|\Gamma(x) \cap U_{j}\right| \geqslant(1-\delta) d_{i, j} p m
$$

DEmonstraÇ̃̃o. Vamos definir a seqüência de quádruplas $\left\{\left(V_{1}^{(i)}, V_{2}^{(i)}, V_{3}^{(i)}, V_{4}^{(i)}\right): i \geqslant 0\right\}$ da seguinte forma:

$$
\left(V_{1}^{(0)}, V_{2}^{(0)}, V_{3}^{(0)}, V_{4}^{(0)}\right)=\left(V_{1}, V_{2}, V_{3}, V_{4}\right) .
$$

Suponha que estão definidos $\left(V_{1}^{(t)}, V_{2}^{(t)}, V_{3}^{(t)}, V_{4}^{(t)}\right)$. Se todo vértice $x$ nesses conjuntos satisfazem a equação (31) para $U_{j}=V_{j}^{(t)}$, então estamos feitos. Caso contrário, vamos supor que existem $i, j \in[4]$ e $x \in V_{i}^{(t)}$ tais que

$$
\left|\Gamma(x) \cap V_{j}^{(t)}\right|<(1-\delta) d_{i, j} p m .
$$

Então, fazemos

$$
\begin{aligned}
V_{j}^{(t+1)} & =V_{j}^{(t)}, \\
V_{i}^{(t+1)} & =V_{i}^{(t)} \backslash\{x\}, \mathrm{e} \\
v_{t} & =x .
\end{aligned}
$$

Agora, vamos supor, sem perda de generalidade, que em algum momento $r$ temos $\left|V_{1}^{(r)}\right|<$ $(1-3 \varepsilon) m$ e que, para todo $j \neq 1$, temos $\left|V_{j}^{(r)}\right| \geqslant(1-3 \varepsilon) m$. 
Pela construção da seqüência, para todo $x \in V_{1} \backslash V_{1}^{(r)}$ existem $t_{x} \in\{0, \ldots, r-1\}$ e $j_{x} \in[4]$ tais que $x=v_{t_{x}} \mathrm{e}$

$$
\left|\Gamma(x) \cap V_{j_{x}}^{\left(t_{x}\right)}\right|<(1-\delta) d_{1, j_{x}} p m .
$$

Como $\left|V_{1} \backslash V_{1}^{(r)}\right| \geqslant 3 \varepsilon m$, existem $j \in\{2,3,4\}$ e $X \subseteq V_{1} \backslash V_{1}^{(r)}$ com $|X| \geqslant \varepsilon m$ tais que

$$
\left|\Gamma(x) \cap V_{j}^{\left(t_{x}\right)}\right|<(1-\delta) d_{1, j} p m,
$$

para todo $x \in X$. Claramente, $\left|\Gamma(x) \cap V_{j}^{(r)}\right| \leqslant\left|\Gamma(x) \cap V_{j}^{\left(t_{x}\right)}\right|$. Dessa forma,

$$
e\left(X, V_{j}^{(r)}\right)<(1-\delta) d_{1, j} p m|X|
$$

donde concluímos que

$$
d_{p}\left(X, V_{j}^{(r)}\right)<(1-\delta) d_{1, j} \frac{m}{\left|V_{j}^{(r)}\right|}<\frac{1-\delta}{1-3 \varepsilon} d_{1, j} \leqslant\left(1-\frac{\varepsilon}{\rho_{0}(1-3 \varepsilon)}\right) d_{1, j},
$$

e, como $(\varepsilon /(1-3 \varepsilon))\left(d_{1, j} / \rho_{0}\right) \geqslant \varepsilon /(1-3 \varepsilon)>\varepsilon$, temos que

$$
d_{p}\left(X, V_{j}^{(r)}\right)<d_{1, j}-\varepsilon
$$

contrariando a $\varepsilon$-regularidade dos pares.

Agora, vamos mostrar que esses $U_{i}$ 's dados pelo resultado acima expandem.

Proposição 45. Para todos $i, j \in\{1,2,3,4\}$ o grafo bipartido induzido pelos conjuntos $U_{i}$ e $U_{j}$ dados pela proposição anterior é $\left((1-2 \delta) d_{i, j} m / f, f\right)$-expansivel, para todo $0<f \leqslant$ $\left(\delta \rho_{0} \alpha / A\right)^{2} d$.

DemonstraçÃo. Suponha $X \subseteq U_{i}$ tal que $|X| \leqslant(1-2 \delta) d_{i, j} m / f$. Pomos $Y=\Gamma(X) \subseteq$ $U_{j}$, para $j \neq i$, e supomos que $|Y|<f|X|$.

Por um lado, pela $(p, A)$-uniformidade, temos

$$
e(X, Y) \leqslant p|X||Y|+A(d|X||Y|)^{1 / 2}<p|X|(1-2 \delta) d_{i, j} m+A(d|X||Y|)^{1 / 2},
$$

e por outro lado, de (31) temos

$$
e(X, Y) \geqslant(1-\delta) d_{i, j} p m|X| .
$$


Dessas duas desigualdades, tiramos que $\left(\delta d_{i, j} p m|X|\right)^{2}<A^{2} d|X||Y|$, ou seja

$$
|Y|>\frac{\delta^{2} d_{i, j}^{2} \alpha^{2}}{A^{2}} d|X| \geqslant\left(\frac{\delta d_{i, j} \alpha}{A}\right)^{2} d|X|
$$

que juntamente com $|Y|<f|X|$ contradiz a cota superior $\left(\delta \rho_{0} \alpha / A\right)^{2} d$ para $f$.

Definimos

$$
\ell=\left\lfloor 2 \lg \left(\frac{(1-2 \delta) \rho_{0} m}{4}\right)+1\right\rfloor \quad \text { e } \quad b=\left\lfloor 2(1-2 \delta) \rho_{0} m\right\rfloor-1 .
$$

O seguinte lema diz que existem muitos caminhos longos entre vértices de $V_{1}$ e de $V_{4} \mathrm{em}$ $\mathbf{P}^{(m)}$.

Lema 46. Seja $s \in \mathbb{N}$ tal que $2 \ell \leqslant s \leqslant 2 \ell+b$. Então, existem $X \subseteq V_{1}$ e $Y \subseteq V_{4} d e$ cardinalidade $|X|,|Y| \geqslant \varepsilon m$, tais que todo vértice de $X$ é ligado a todo vértice de $Y$ por uma caminho de comprimento $s$ contido em $\bigcup_{i=1}^{4} V_{i}$.

DemonstraÇÃo. Fixado $\mathbf{P}^{(m)}$, aplicamos a Proposição 44 e obtemos $U_{i} \subseteq V_{i}$, para todo $i \in[4]$. Sabemos, pela Proposição 45 que tais $U_{i}$ 's são dois-a-dois $\left((1-2 \delta) \rho_{0} m / f, f\right)$ expansíveis para todo $0<f \leqslant 4$.

Temos que $\left(U_{2}, U_{3}\right)$ é $\left((1-2 \delta) \rho_{0} m / 2,2\right)$-expansível. Aplicamos o Lema 42 a esse par e assim, sabemos que esse par contém um caminho $P=x_{0}, \ldots, x_{b}$ de comprimento $b$, onde $b$ é como na equação (32) acima.

Supomos, sem perda de generalidade, que $x_{0} \in U_{2}$ e vamos considerar o par $\left(U_{2}, U_{1}\right)$, o qual é $\left((1-2 \delta) \rho_{0} m / 4,4\right)$-expansível. Aplicando o Lema 43 para $\ell$ dado em (32), temos que

$$
\left|\Gamma^{(\ell)}\left(x_{0}\right) \cap U_{1}\right|>2^{\lfloor\ell / 2\rfloor}>\frac{(1-2 \delta) \rho_{0} m}{8} .
$$

Agora, tomamos $t=s-2 \ell$ e $x_{t} \in V(P)$. Como $x_{t} \in U_{i}$, para algum $i \in\{2,3\}$, e o par $\left(U_{4}, U_{i}\right)$ é $\left((1-2 \delta) \rho_{0} m / 4,4\right)$-expansível, temos pelo mesmo argumento do parágrafo anterior que

$$
\left|\Gamma^{(\ell)}\left(x_{t}\right) \cap U_{4}\right|>\frac{(1-2 \delta) \rho_{0} m}{8} .
$$

Pela escolha de $\varepsilon$ temos $(1-2 \delta) \rho_{0}>8 \varepsilon$. Então para $(i, j)=(0,1)$ e $(i, j)=(t, 4)$ temos

$$
\left|\Gamma^{(\ell)}\left(x_{i}\right) \cap U_{j}\right|>\frac{(1-2 \delta) \rho_{0} m}{8}>\varepsilon m .
$$


Logo, tomamos

$$
\begin{aligned}
& X=\Gamma^{(\ell)}\left(x_{0}\right) \cap U_{1}, \\
& Y=\Gamma^{(\ell)}\left(x_{t}\right) \cap U_{4} .
\end{aligned}
$$

Dessa forma todo vértice de $X$ é ligado a todo vértice de $Y$ por um caminho de comprimento $s=2 \ell+t$, para $\ell=\left\lfloor 2 \lg \left((1-2 \delta) \rho_{0} m / 4\right)+1\right\rfloor$ e $0 \leqslant t \leqslant\left\lfloor 2\left((1-2 \delta) \rho_{0} m\right\rfloor-1\right.$.

2.3. Grafos uniformes são esparsos. Antes de podermos aplicar Lema de Regularidade, devemos verificar o seguinte fato que, em particular, diz que grafos $(p, A)$-uniformes de ordem $n$ são $(\eta, 1+\eta, p)$-esparsos, para todo $\eta>0$ dado que $d=p n$ é suficientemente grande.

Proposição 47. Dados $A>0$ e $0<p, \eta \leqslant 1$ seja $G^{n}$ um grafo $(p, A)$-uniforme e ponha $d=$ pn. Se $\eta^{4} d \geqslant A^{2}$ e $\eta d \geqslant 1-\eta$, então $G^{n}$ é $\eta$-uniforme, ou seja, para todos $U, W \subseteq V\left(G^{n}\right)$ disjuntos com $\geqslant \eta n$ vértices cada então

$$
\left|e_{G^{n}}(U, W)-p\right| U|| W|| \leqslant \eta p|U||W|
$$

DemonstraÇÃo. Basta notar que $A(d|U||W|)^{1 / 2}=A n^{1 / 2}(p|U||W|)^{1 / 2}$ e que $A n^{1 / 2} \leqslant$ $\eta^{2}(p n)^{1 / 2} n^{1 / 2}=\eta^{2} p^{1 / 2} n=\eta p^{1 / 2} \eta n \leqslant \eta p^{1 / 2}(|U||W|)^{1 / 2}$. Portanto,

$$
A(d|U||W|)^{1 / 2} \leqslant \eta p^{1 / 2}(|U||W|)^{1 / 2}(p|U||W|)^{1 / 2}=\eta p|U||W| .
$$

\section{Demonstração do teorema principal}

O nosso próximo passo é aplicar o Lema de Regularidade a um grafo $(p, A)$-uniforme com o intuito de acharmos um $t$-upla de subconjuntos dois-a-dois disjuntos e $\varepsilon$-regulares de densidade positiva.

Vamos supor dadas as constantes positivas $A \in \mathbb{R}$ e $q \in \mathbb{N}$. Seja $H$ um grafo de grau máximo $\Delta=\Delta(H)$ e seja $t=t(H, q) \in \mathbb{N}$ uma constante que definiremos mais tarde. Vamos definir as constantes

$$
\varrho=\frac{1}{24(t-1)}, \quad \text { e } \quad \rho_{0}=\frac{\varrho}{q}
$$


Também, definimos

$$
D=2, \quad \varepsilon=\min \left\{\frac{1}{192(t-1)}, \frac{\rho_{0}}{2\left(5+3 \rho_{0}\right)}, \frac{1}{\Delta+1}\right\} \quad \text { e } \quad k_{0}=\varepsilon^{-1},
$$

e tomamos

$$
\eta=\eta\left(\varepsilon, D, k_{0}, q\right) \quad \text { e } \quad K_{0}=K_{0}\left(\varepsilon, D, k_{0}, q\right)
$$

as constantes dadas pelo Lema 10. Assumimos que $\eta<1 / 24(t-1)$ e tomamos

$$
\alpha=\frac{1}{2 K_{0}} \quad \text { e } \quad d_{0}=\max \left\{A^{2} \eta^{-4}, \frac{1-\eta}{\eta}, \frac{1}{\left(\rho_{0}-\varepsilon\right) \varepsilon \alpha}, 4\left(\frac{\alpha \rho_{0} \delta}{A}\right)^{-2}\right\} .
$$

Seja $G=G^{n}$ um grafo $(p, A)$-uniforme, onde $p=p(n)$ é tal que $d=p n \geqslant d_{0}$. Aplicamos, a esse subgrafo, o Lema 10 da página 43 para $\varepsilon, k_{0}$ e $D$ como acima e para

$$
G_{a}=G\left[E_{a}\right]
$$

onde $E_{1}, \ldots, E_{q}$ é uma coloração arbitrária de $E(G)$. Seja $\Pi=\left(V_{0}, V_{1}, \ldots, V_{k}\right)$ a partição $\left(\varepsilon, k, G_{a} ; p\right)$-regular, para toda cor $a \in[q]$, dada por esse lema.

Vamos mostrar que na partição $\Pi$ mais que $(1-1 /(t-1))\left(k^{2} / 2\right)$ pares $\left(V_{i}, V_{j}\right)$ são $\left(\varepsilon, G_{a} ; p\right)$ regulares $(\forall a \in[q])$ e têm densidade

$$
d_{G, p}\left(V_{i}, V_{j}\right) \geqslant \varrho
$$

Contamos o número de arestas de $G$ que não pertencem a pares $\left(\varepsilon, G_{a} ; p\right)$-regulares de densidade pelo menos $\varrho$ em $G$. Essas arestas se classificam num dos quatro tipos abaixo:

- cada um dos no máximo $\varepsilon\left(\begin{array}{l}k \\ 2\end{array}\right)$ pares irregulares contém no máximo $(1+\eta) p m^{2}$ arestas e temos, no total,

$$
\leqslant \varepsilon\left(\begin{array}{l}
k \\
2
\end{array}\right)(1+\eta) p m^{2}<\varepsilon(1+\eta) \frac{n^{2} p}{2} \text { arestas; }
$$

- entre os pares $\varepsilon$-regulares com densidade menor que $\varrho$ há menos que $\varrho p m^{2}$ arestas e temos

$$
\leqslant\left(\begin{array}{l}
k \\
2
\end{array}\right) \varrho p m^{2}<\varrho(1+\eta) \frac{n^{2} p}{2} \text { arestas; }
$$


- cada $V_{i}$, para $i \neq 0$, contém no máximo $(1+\eta) p\left(\begin{array}{c}m \\ 2\end{array}\right)$ arestas (veja as Observações 5 e 6 na página 43). Portanto, no total temos

$$
<k(1+\eta) p\left(\begin{array}{c}
m \\
2
\end{array}\right) \leqslant(1+\eta) \frac{n^{2} p}{2 k} \text { arestas; }
$$

- resta-nos contar o número de arestas com pelo menos um extremo em $V_{0}$. Escrevemos $U=V_{1} \cup V_{0}$. Assim,

$$
e\left(G\left[V_{0}\right]\right) \leqslant e(G[U]) \leqslant(1+\eta) p\left(\begin{array}{c}
m+\varepsilon n \\
2
\end{array}\right) \leqslant\left(\varepsilon+\frac{1}{k}\right)^{2}(1+\eta) \frac{n^{2} p}{2}
$$

e o número de arestas que ligam vértices de $V_{0}$ aos vértices das outras classes é

$$
<\sum_{i=2}^{k} e_{G}\left(U, V_{i}\right) \leqslant \sum_{i=2}^{k}(1+\eta) p(m+\varepsilon n)\left|V_{i}\right|<\left(\varepsilon+\frac{1}{k}\right)(1+\eta) p n^{2}
$$

Portanto, temos no máximo

$$
3\left(\varepsilon+\frac{1}{k}\right)(1+\eta) \frac{n^{2} p}{2}
$$

arestas com pelo menos um extremo na classe excepcional.

Somando, temos menos que

$$
\left(4 \varepsilon+\frac{4}{k_{0}}+\varrho\right)(1+\eta) \frac{n^{2} p}{2} \leqslant(8 \varepsilon+\varrho)(1+\eta) \frac{n^{2} p}{2}
$$

arestas que não estão em pares regulares densos.

Vamos supor que na partição П no máximo $(1-1 /(t-1))\left(k^{2} / 2\right)$ pares $\left(V_{i}, V_{j}\right)$ são $\left(\varepsilon, G_{a} ; p\right)$ regulares e têm densidade pelo menos $\varrho$ em $G$. Cada par desses induz no máximo $(1+\eta) p m^{2} \leqslant$ $(1+\eta) p(n / k)^{2}$ arestas, portanto, no máximo

$$
\left(1-\frac{1}{t-1}\right) \frac{k^{2}}{2}(1+\eta) \frac{n^{2} p}{k^{2}}
$$


arestas pertencem a esses pares regulares densos. Portanto, somando as equações (37) e (38), temos

$$
\begin{aligned}
e(G) & <\left(1-\frac{1}{(t-1)}+8 \varepsilon+\varrho\right)(1+\eta) \frac{n^{2} p}{2} \\
& \leqslant\left(1-\frac{1}{(t-1)}+8 \varepsilon+\varrho+\eta\right) \frac{n^{2} p}{2} \\
& \leqslant\left(1-\frac{5}{6(t-1)}\right) \frac{n^{2} p}{2} .
\end{aligned}
$$

Notemos que esse número contraria a equação (29), página 94, quando $d \geqslant 13(t-1)^{2}$. Nesse caso, quando $d$ é suficientemente grande, definimos um grafo $R$ com $k$ vértices, um para cada parte não excepcional de $\Pi$, e ligamos dois vértices se os pares correspondentes são $\left(\varepsilon, G_{a} ; p\right)$-regulares, para todo $a \in[q]$, de $p$-densidade em $G$ pelo menos $\varrho$. Pelos nossos cálculos

$$
e(R)>\left(1-\frac{1}{t-1}\right) \frac{k^{2}}{2}
$$

então pelo Teorema de Turán $K^{t} \subseteq R$, ou seja, temos uma $t$-upla de classes de $\Pi$ duas-a-duas $\left(\varepsilon, G_{a} ; p\right)$-regulares, para todo $a \in[q]$ e de $p$-densidade pelo menos $\varrho$.

Para prosseguirmos com a prova, consideramos as constantes dadas por (33), (34), (35) e (36) acima e tomamos

$$
k_{1}=v(H)+4 e(H) \quad \text { e } \cdot t=r\left(K^{k_{1}} ; q\right),
$$

onde $r\left(K^{k_{1}} ; q\right)$ é o número de ramsey usual para $q$ cores. Vamos provar o Teorema 40 para as seguintes escolhas de constantes

$$
C_{0}=\max \left\{d_{0}, 13(t-1)^{2}\right\}, \quad C_{1}=5 \quad \text { e } \quad C_{2}=(1-2 \delta) \alpha \rho_{0}
$$

Resumindo, $G=G^{n}$ é um grafo $(p, A)$-uniforme, onde $p=p(n)$ é tal que $d=p n \geqslant C_{0}$, e $E(G)=E_{1} \cup \cdots \cup E_{q}$ uma $q$-aresta-coloração de $G$. Pelo Lema 10 , o grafo $G$ admite uma partição $\left(\varepsilon, k, G_{a}, p\right)$-regular $\Pi=\left(V_{0}, V_{1}, \ldots, V_{k}\right)$, para algum $k_{0} \leqslant k \leqslant K_{0}$, para todo $a \in[q]$ e onde $G_{a}=G\left[E_{a}\right]$. Tomamos $m=\left|V_{i}\right|$, para todo $i \neq 0$, e pela escolha de $\alpha$ temos $m \geqslant \alpha n$.

Seja $s \in \mathbb{N}$ tal que

$$
5 \lg n \leqslant s-2 \leqslant(1-2 \delta) \alpha \rho_{0} n .
$$


Assim,

$$
\left.4 \lg \left((1-2 \delta) \rho_{0} m\right)\right) \leqslant s-2 \leqslant 2(1-2 \delta) \rho_{0} m,
$$

portanto, $2 \ell \leqslant s-2 \leqslant 2 \ell+b$, onde $\ell$ e $b$ são como em (32).

Tomemos o grafo $R$ pondo $V(R)=\left\{V_{1}, \ldots, V_{k}\right\}=\Pi \backslash\left\{V_{0}\right\}$ e temos uma aresta $\left\{V_{i}, V_{j}\right\} \in$ $E(R)$ se, e somente se, o par $\left(V_{i}, V_{j}\right)$ é $\left(\varepsilon, G_{a} ; p\right)$-regular, para todo $a \in[q]$, de $p$-densidade $\geqslant \varrho$ em $G$. Pelas escolhas das constantes, por (39) e pelo Teorema 6, página 29, temos uma cópia $K^{t}$ do grafo completo de $t$ vértices em $R$. Definimos nesse $K^{t}$ a seguinte $q$-aresta-coloração:

$$
\operatorname{cor}\left(V_{i}, V_{j}\right)=\min \left\{a \in[q]: d_{G_{a}, p}\left(V_{i}, V_{j}\right) \geqslant \rho_{0}\right\}
$$

Observamos que tal aresta-coloração está bem definida. De fato, $d_{G, p}\left(V_{i}, V_{j}\right) \geqslant \varrho$ para todos $\left\{V_{i}, V_{j}\right\} \in E\left(K_{t}\right)$, portanto, para alguma cor $a \in[q]$ temos $d_{G_{a}, p}\left(V_{i}, V_{j}\right) \geqslant \varrho / q=\rho_{0}$. Pela escolha de $t$ temos $K^{k_{1}} \subseteq K^{t}$ monocromático.

Associamos cada vértice $x \in V(H)$ com um, e só um, vértice $V_{x} \in V\left(K^{k_{1}}\right)$. Restam $4 e(H)$ vértices em $V\left(K^{k_{1}}\right)$. Para cada aresta $e=x y \in E(H)$ fixamos uma quádrupla $\mathrm{P}_{e}^{(m)}=$ $\left(V_{i}^{(e)}\right)_{i=1}^{4}$ de vértices do $K^{k_{1}}$. Dessa forma, estamos nas hipóteses do Lema 46, logo para cada $e=x y \in E(H)$ temos $X^{e} \subseteq V_{1}^{e}$ e $Y^{e} \subseteq V_{4}^{e}$ com $\left|X^{e}\right|,\left|Y^{e}\right| \geqslant \varepsilon m$ e todo vértice de $\left|X^{e}\right|$ está ligado a todo vértice de $\left|Y^{e}\right|$ por um caminho de comprimento $s-2$.

Agora, vamos descrever a imersão de $H^{(s)}$ em $G$. Antes, chamamos a atenção para o seguinte fato, que é o análogo para $p$-densidade da Afirmação 1, página 39:

Observação 8. (i) Se $(U, W)$ é um par $(\varepsilon ; p)$-regular com densidade $d=d(U, W)$, então para qualquer $Y \subseteq W \operatorname{com}|Y| \geqslant \varepsilon|W|$, temos $\geqslant(1-\varepsilon)|U|$ vértices $x \in U$ tais que $|\Gamma(x) \cap Y| \geqslant$ $(d-\varepsilon) p|Y|$. Além disso, (ii) se $\left(U, W_{j}\right)_{j=1}^{\Delta}$ são pares $(\varepsilon ; p)$-regular com densidade pelo menos $d>0$ e $Y_{j} \subseteq W_{j}$ são subconjuntos com pelo menos $\varepsilon\left|W_{j}\right|$ elementos, então existem pelo menos $(1-\Delta \varepsilon)|U|$ vértices $u \in U$ tais que $\Gamma(u) \cap Y_{j} \neq \emptyset$ para todo $j \in[\Delta]$.

A verificação da primeira das afirmações acima é imediata a partir da Afirmação 1. Para a verificação da segunda, usamos a primeira $\Delta$ vezes e ficamos com $\geqslant(1-\Delta \varepsilon) m$ vértices com pelo menos $\left(\rho_{0}-\varepsilon\right) p\left|Y_{j}\right|$ vizinhos em cada $Y_{j}$. 
Voltando a nossa demonstração, lembramos que para cada vértice $x \in V(H)$ temos $V_{x} \in$ $V\left(K^{k_{1}}\right)$, para cada aresta $e=x y \in E(H)$ temos uma quádrupla $\mathbf{P}_{e}^{(m)}$ de vértices do $K^{k_{1}} \mathrm{e}$ temos $X^{e} \subseteq V_{1}^{e} \in \mathbf{P}_{e}^{(m)}$ e $Y^{e} \subseteq V_{4}^{e} \in \mathbf{P}_{e}^{(m)} \operatorname{com}\left|X^{e}\right|,\left|Y^{e}\right| \geqslant \varepsilon m$.

Vamos tomar sobre $V(H)$ uma ordem total $\prec$ qualquer. Definimos

$$
\Lambda=\{(x, y) \in V(H) \times V(H): x y \in E(H) \text { e } x \prec y\}
$$

Usando os fatos da Observação 8, para cada $x \in V(H)$ escolhemos $\bar{x} \in V_{x} \in V\left(K^{k_{1}}\right)$ de modo que

$$
\begin{aligned}
& \left|\Gamma(\bar{x}) \cap X^{e}\right| \geqslant\left(\rho_{0}-\varepsilon\right) p \varepsilon m \text { para todo } e=(x, y) \in \Lambda, \\
& \left|\Gamma(\bar{x}) \cap Y^{e}\right| \geqslant\left(\rho_{0}-\varepsilon\right) p \varepsilon m \text { para todo } e=(y, x) \in \Lambda .
\end{aligned}
$$

Notamos que $\left(\rho_{0}-\varepsilon\right) p \varepsilon m \geqslant\left(\rho_{0}-\varepsilon\right) d \varepsilon \alpha>1$, pela escolha de $d \geqslant C_{0}$; e notamos que existem $\geqslant(1-\Delta \varepsilon) m>0$ (pela escolha de $\varepsilon<1 /(\Delta+1))$ tais $\bar{x}$.

Agora, se $x y \in E(H)$, então $\bar{x}$ e $\bar{y}$ estão ligados por um caminho de comprimento $C_{1} \lg n \leqslant$ $s \leqslant C_{2} n$.

\section{Grafos $(p, A)$-uniformes}

Nesta seção vamos mostrar exemplos de grafos $(p, A)$-uniforme com tamanho linear no número de vértices.

4.1. Grafos aleatórios. Vejamos uma prova probabilística da existência de grafos uniformes.

Lema 48 (Haxell et al., 1995a). Para todo $0<p=p(n)<1$ tal que $d=d(n)=p n \geqslant 1$, quase certamente $G_{n, p}$ é $(p, 20)$-uniforme.

Demonstração. Primeiro, fixamos $U$ e $W$ subconjunto de vértices disjuntos com $u \leqslant$ $w \leqslant d u$, onde $u=|U|, w=|W|$ e $d=p n$. Vamos escrever $\mu=p u w$ e $\lambda=A(d u w)^{1 / 2}$, lembrando que $A=20$.

Notamos que $\mu=\lambda^{2} / A^{2} n$ e pela desigualdade de Chernoff, equação (7) na página 33, temos que

$$
\mathbb{P}\left\{e_{G_{p}}(U, W) \leqslant \mu-\lambda\right\} \leqslant \exp \left\{\frac{-\lambda^{2}}{2 \mu}\right\} \leqslant \exp \left\{\frac{-A^{2} n}{2}\right\}
$$


O número de pares de subconjuntos $U$ e $W$ como acima é menor que $4^{n}=\mathrm{e}^{\ln (4) n}$, logo o número esperado de tais pares que induzem no máximo $\mu-\lambda$ arestas é menor que

$$
\exp \left\{\ln (4) n-\frac{A^{2}}{2} n\right\}=o(1)
$$

Agora, vamos mostrar que o número esperado de pares de subconjuntos que induzem pelo menos $\mu+\lambda$ arestas é pequeno. Vamos considerar dois casos: $\lambda \leqslant \mathrm{e}^{2} \mu$ e $\lambda>\mathrm{e}^{2} \mu$.

Supomos que $\lambda \leqslant \mathrm{e}^{2} \mu$. Então

$$
\mathbb{P}\left\{e_{G_{p}}(U, W) \geqslant \mu+\lambda\right\} \leqslant \exp \left\{\frac{-\lambda^{2}}{2(\mu+\lambda / 3)}\right\} \leqslant \exp \left\{\frac{-\lambda^{2}}{2 \mu\left(1+\mathrm{e}^{2} / 3\right)}\right\} \leqslant \exp \left\{\frac{-3 A^{2}}{2\left(\mathrm{e}^{2}+3\right)} n\right\},
$$

e o número esperado de pares que induzem muitas arestas é no máximo

$$
\exp \left\{\ln (4) n-\frac{3 A^{2}}{2\left(\mathrm{e}^{2}+3\right)} n\right\} \leqslant \exp \{-56 n\}=o(1) .
$$

Para finalizar, supomos que $\lambda>\mathrm{e}^{2} \mu$. Usando a desigualdade de Chernoff temos

$$
\mathbb{P}\left\{e_{G_{p}}(U, W) \geqslant \mu+\lambda\right\} \leqslant \exp \left\{-\mu \varphi\left(\frac{\lambda}{\mu}\right)\right\} \leqslant \exp \left\{\ln \left(1+\frac{\lambda}{\mu}\right)^{-\mu\left(1+\frac{\lambda}{\mu}\right)}+\lambda\right\},
$$

portanto, temos que

$$
\mathbb{P}\left\{e_{G_{p}}(U, W) \geqslant \mu+\lambda\right\} \leqslant\left(1+\frac{\lambda}{\mu}\right)^{-\mu\left(1+\frac{\lambda}{\mu}\right)} \mathrm{e}^{\lambda} \leqslant\left(\frac{\mathrm{e}}{\lambda / \mu}\right)^{\lambda}=x^{B x},
$$

onde $x=\mathrm{e} /(\lambda / \mu)$ e $B=A^{2} n / \mathrm{e}$. De $u \leqslant w \leqslant d u$ temos, para $r=u+w$, que $(d u w)^{1 / 2} \geqslant$ $d r /(d+1) \geqslant r / 2$, e como $x^{x}$ é decrescente para $0<x \leqslant 1 /$ e, ficamos com

$$
\mathbb{P}\left\{e_{G_{p}}(U, W) \geqslant \mu+\lambda\right\} \leqslant\left(\frac{\mathrm{e}}{\lambda / \mu}\right)^{\lambda}=\left(\frac{\mathrm{e}(d u w)^{1 / 2}}{A n}\right)^{A(d u w)^{1 / 2}} \leqslant\left(\frac{\mathrm{e} r}{2 A n}\right)^{A r / 2} .
$$

Escrevemos $\mathbb{P}_{u, w}$ para $\mathbb{P}\left\{e_{G_{p}}(U, W) \geqslant \mu+\lambda\right\}$, assim temos que o número esperado de pares que excedem $\mu+\lambda$ arestas é no máximo

$$
\sum_{r=2}^{n} \sum_{u=1}^{r / 2}\left(\begin{array}{l}
n \\
r
\end{array}\right)\left(\begin{array}{l}
r \\
u
\end{array}\right) \mathbb{P}_{u, r-u} \leqslant \sum_{r=2}^{n} \sum_{u=1}^{r / 2}\left(\begin{array}{l}
r \\
u
\end{array}\right)\left(\frac{r}{4 n}\right)^{r} \leqslant \sum_{r=2}^{n}\left(\frac{r}{2 n}\right)^{r} \leqslant 2 n^{-2}=o(1) .
$$

Ou seja, o número esperado de subconjuntos $U$ e $W$ disjuntos com $|U| \leqslant|W| \leqslant d|U|$ e tais que

$$
\left|e_{G_{p}}(U, W)-p\right| U|| W|| \geqslant A(d|U||W|)^{1 / 2}
$$


é $o(1)$ e concluímos a afirmação do lema aplicando a desigualdade de Markov.

4.2. Grafos de Ramanujan. Agora, vamos ver exemplos explicitamente construídos de grafos uniformes.

Os grafos de Ramanujan formam outra classe de grafos $(p, A)$-uniformes. Sejam $p$ e $q$ primos tais que $p, q \equiv 1(\bmod 4)$ e $p$ não é um resíduo quadrático em $\mathbb{Z}_{p}$. Denotamos por $X^{p, q}$ o grafo construído por Lubotzky et al. (1988), o qual é de ordem $n=q\left(q^{2}-1\right) / 2$ e $d$ regular, para $d=p+1$. (Trata-se de um certo grafo de Cayley, definido a partir de $\operatorname{PGL}\left(2, \mathbb{Z}_{q}\right)$; não entraremos em detalhes.)

Lubotzky et al. (1988) provaram que os autovalores $0 \leqslant\left|\lambda_{n-1}\right| \leqslant \cdots \leqslant\left|\lambda_{0}\right|=d$ da matriz de adjacências de $X^{p, q}$ satisfazem a propriedade de Ramanujan, ou seja,

$$
\left|\lambda_{i}\right| \leqslant 2(d-1)^{1 / 2} \text { para todo } i \geqslant 1 \text {. }
$$

As técnicas de Alon e Milman (1985) (veja também Alon e Spencer, 1992, Capítulo 9, Corolário 2.5) são suficiente para deduzir a partir de $\lambda_{1}$ que para todo $U, W \subseteq V\left(X^{p, q}\right)$ disjuntos vale $|e(U, W)-(d / n)| U\|W\| \leqslant 2\{(d-1)|U||W|\}^{1 / 2}$, logo, $X^{p, q}$ é $(d / n, 2)$-uniforme.

\section{Considerações finais}

Vojtech Rödl sugeriu-nos a seguinte leitura para a conjectura de Pak:

Conjectura 49. Para todo $\Delta \in \mathbb{N}$ e para todo grafo $H$ com grau máximo $\Delta(H) \leqslant \Delta$ existe uma constante positiva $C=C(\Delta)$ tal que $\hat{r}\left(H^{(s)}\right) \leqslant C e(H) s$, para todo $s>\log v(H)$.

O nosso resultado diz que que existe $s_{0}=s_{0}(H)$ tal que $\hat{r}\left(H^{(s)}\right) \leqslant C s$, para todo $s>s_{0}$. Tal $s_{0}$ deve ser suficientemente grande de modo que, para todo $s>s_{0}$, podemos aplicar o Lema de Regularidade num grafo com $O\left(v\left(H^{(s)}\right)\right)$ vértices e garantir uma cópia monocromática do $H^{(s)}$ nesse grafo.

Alon e Chung (1988) usam $X^{p, q}$ para mostrar que para todo $\varepsilon>0$ e todo $n \geqslant 1$ existe um grafo $G$, que pode ser explicitamente construído, com $O(m / \varepsilon)$ vértices e grau máximo $O\left(1 / \varepsilon^{2}\right)$, tal que mesmo após a remoção de $(1-\varepsilon) v(G)$ vértices e de $(1-\varepsilon) e(G)$ arestas, o 
grafo resultante contém um caminho de comprimento $n$. Esse resultado implica na construção explicita de grafos que mostram que o número de ramsey-aresta para $P^{n}$ é linear.

O resultado de Friedman e Pippenger (1987), inspirado no trabalho de Alon e Chung, também usa os grafos de Ramanujan para mostrar explicitamente grafos que são ramsey para árvores de grau limitado e com tamanho linear no número de vértices da árvore.

Agora, listamos algumas conjecturas para números de ramsey-aresta fora da diagonal. Ke (1993) mostrou que para $n$ suficientemente grande valem as seguintes desigualdades

$$
c_{1} n^{3} \leqslant \hat{r}\left(K^{n}, P^{n}\right) \leqslant c_{2} n^{3} \log n,
$$

onde $c_{1}$ e $c_{2}$ são constantes absolutas;

$$
c_{1} n^{3} \leqslant \hat{r}\left(K^{n}, T_{n, \Delta}\right) \leqslant c_{2} n^{3} \log n,
$$

onde $c_{1}$ é uma constante absoluta e $c_{2}=c_{2}(\Delta)$ é constante;

$$
c_{1} m^{2} n \leqslant \hat{r}\left(K^{m}, T_{n, \Delta}\right) \leqslant c_{2} m^{2} n \log n,
$$

com $m^{\beta}<n<m^{\gamma}$ e onde $c_{1}$ e $c_{2}=c_{2}(\Delta, \beta, \gamma)$ são constantes, e conjecturou

Conjectura 50 (Ke, 1993).

$$
\begin{gathered}
\hat{r}\left(K^{n}, P^{n}\right) \leqslant c n^{3}, \\
\hat{r}\left(K^{n}, T_{n, \Delta}\right) \leqslant c \Delta n^{3}, \\
\hat{r}\left(K^{m}, T_{n, \Delta}\right) \leqslant c \Delta m^{2} n .
\end{gathered}
$$

Conjectura 51 (Pikhurko, 2001). Para todo grafo $G$ com número cromático $\chi(G) \geqslant 4$

$$
\hat{r}\left(K^{1, n}, G\right)=(1+o(1)) \frac{\chi(G)(\chi(G)-2)}{2} n^{2} .
$$




\section{Referências Bibliográficas}

Alon, N. e Chung, F. (1988). Explicit construction of linear sized tolerant networks. Discrete Mathematics, 72:15-19.

Alon, N. e Milman, V. (1985). $\lambda_{1}$, isoperimetric inequalities and superconcentrators. $J$. Combin. Theory Ser. B, 38:73-88.

Alon, N. e Spencer, J. H. (1992). The probabilistic method. John Wiley \& Sons Inc., New York.

Beck, J. (1983). On size Ramsey number of paths, trees, and circuits. I. J. Graph Theory, $7(1): 115-129$.

Beck, J. (1990). On size Ramsey number of paths, trees and circuits. II. Em Mathematics of Ramsey theory, páginas 34-45. Springer, Berlin.

Bollobás, B. (1998). Modern Graph Theory. Springer-Verlag, New York.

Bollobás, B., Donadelli, J., Kohayakawa, Y., e Schelp, R. H. (2002). On $\left(C^{\ell}, H\right)$-ramsey minimal graphs. Submetido em Janeiro de 2002.

Burr, S. A. (1986). Some Ramsey-infinite graphs in the diagonal case. Ars Combin., 21:173178.

Burr, S. A., Erdős, P., Faudree, R. J., Rousseau, C. C., e Schelp, R. H. (1980). An extremal problem in generalized Ramsey theory. Ars Combin., 10:193-203.

Burr, S. A., Erdős, P., Faudree, R. J., Rousseau, C. C., e Schelp, R. H. (1981a). Ramseyminimal graphs for star-forests. Discrete Math., 33(3):227-237.

Burr, S. A., Erdős, P., Faudree, R. J., e Schelp, R. H. (1978). A class of Ramsey-finite graphs. Em Proceedings of the Ninth Southeastern Conference on Combinatorics, Graph Theory, and Computing (Florida Atlantic Univ., Boca Raton, Fla., 1978), páginas 171-180, Winnipeg, Man. Utilitas Math. 
Burr, S. A., Faudree, R. J., e Schelp, R. H. (1985). On graphs with Ramsey-infinite blocks. European J. Combin., 6(2):129-132.

Burr, S. A., Faudree, R. J., Schelp, R. H., Erdős, P., e Rousseau, C. C. (1981b). Ramseyminimal graphs for matchings. Em The theory and applications of graphs (Kalamazoo, Mich., 1980), páginas 159-168. Wiley, New York.

Chvatál, C., Rödl, V., Szemerédi, E., e Trotter, Jr., W. T. (1983). The Ramsey number of a graph with bounded maximum degree. J. Combin. Theory Ser. B, 34(3):239-243.

Diestel, R. (1997). Graph theory. Springer-Verlag, New York.

Donadelli, J. e Kohayakawa, Y. (2000). A density result for random oriented sparse graphs and its relation to a conjecture of Woodall. Submetido em Julho de 2000.

Donadelli, J. and Kohayakawa, Y. (2002). On the size-ramsey number of long subdivisions of graphs. Em preparação.

Erdős, P., Faudree, R. J., Rousseau, C. C., e Schelp, R. H. (1978). The size Ramsey number. Period. Math. Hungar., 9(2-2):145-161.

Erdös, P. e Stone, A. H. (1946). On the structure of linear graphs. Bull. Amer. Math. Soc., 52:1087-1091.

Faudree, R. (1991). Ramsey minimal graphs for forests. Ars Combin., 31:117-124.

Faudree, R. J., Rousseau, C. C., e Schelp, R. H. (1997). Problems in graph theory from Memphis. Em The mathematics of Paul Erdős, II, páginas 7-26. Springer, Berlin.

Feofiloff, P. e Younger, D. H. (1987). Directed cut transversal packing for source-sink connected graphs. Combinatorica, 7(3):255-263.

Frankl, P. e Rödl, V. (1986). Large triangle-free subgraphs in graphs without $K_{4}$. Graphs Combin., 2(2):135-144.

Friedman, J. e Pippenger, N. (1987). Expanding graphs contain all small trees. Combinatorica, $7(1): 71-76$.

Füredi, Z. (1994). Random Ramsey graphs for the four-cycle. Discrete Math., 126(1-3):407410.

Gowers, W. T. (1997). Lower bounds of tower type for Szemerédi's uniformity lemma. Geom. Funct. Anal., 7(2):322-337. 
Graham, R. L. e Rödl, V. (1987). Numbers in Ramsey theory. Em Surveys in combinatorics 1987 (New Cross, 1987), páginas 111-153. Cambridge Univ. Press, Cambridge.

Graham, R. L., Rödl, V., e Ruciński, A. (2000). On graphs with linear Ramsey numbers. J. Graph Theory, 35(3):176-192.

Graham, R. L., Rothschild, B. L., e Spencer, J. H. (1980). Ramsey theory. John Wiley \& Sons Inc., New York.

Haxell, P. E. (1997). Partitioning complete bipartite graphs by monochromatic cycles. $J$. Combin. Theory Ser. B, 69(2):210-218.

Haxell, P. E. e Kohayakawa, Y. (1995). The size-Ramsey number of trees. Israel J. Math., $89(1-3): 261-274$.

Haxell, P. E., Kohayakawa, Y., e Euczak, T. (1995a). The induced size-Ramsey number of cycles. Combin. Probab. Comput., 4(3):217-239.

Haxell, P. E., Kohayakawa, Y., e Luczak, T. (1995b). Turán's extremal problem in random graphs: forbidding even cycles. J. Combin. Theory Ser. B, 64(2):273-287.

Haxell, P. E., Kohayakawa, Y., e Euczak, T. (1996). Turán's extremal problem in random graphs: forbidding odd cycles. Combinatorica, 16(1):107-122.

Janson, S., Luczak, T., e Ruciński, A. (2000). Random graphs. Wiley-Interscience, New York. Ke, X. (1993). The size Ramsey number of trees with bounded degree. Random Structures Algorithms, 4(1):85-97.

Kohayakawa, Y. (1993). The regularity lemma of Szemerédi for sparse graphs. Não publicado.

Kohayakawa, Y. (1997). Szemerédi's regularity lemma for sparse graphs. Em Foundations of computational mathematics (Rio de Janeiro, 1997), páginas 216-230. Springer, Berlin.

Kohayakawa, Y. e Kreuter, B. (1997). Threshold functions for asymmetric Ramsey properties involving cycles. Random Structures Algorithms, 11(3):245-276.

Kohayakawa, Y., Kreuter, B., e Steger, A. (1998). An extremal problem for random graphs and the number of graphs with large even-girth. Combinatorica, 18(1):101-120.

Kohayakawa, Y., Łuczak, T., e Rödl, V. (1996). Arithmetic progressions of length three in subsets of a random set. Acta Arith., 75(2):133-163. 
Kohayakawa, Y., Łuczak, T., e Rödl, V. (1997). On $K^{4}$-free subgraphs of random graphs. Combinatorica, 17(2):173-213.

Kohayakawa, Y. e Rödl, V. (2001). Regular pairs in sparse random graphs I. Submetido.

Kohayakawa, Y., Rödl, V., e Szemerédi, E. (2001). Th size-Ramsey number of graphs of bounded degree. Em preparação.

Komlós, J. e Simonovits, M. (1996). Szemerédi's regularity lemma and its applications in graph theory. Em Combinatorics, Paul Erdös is eighty, Vol. 2 (Keszthely, 1993), páginas 295-352. János Bolyai Math. Soc., Budapest.

Lee, O. (1999). Coberturas de Circuitos em Grafos Mistos. Tese de Doutoramento, Instituto de Matemática e Estatística, USP, São Paulo.

Lubotzky, A., Phillips, R., e Sarnak, P. (1988). Ramanujan graphs. Combinatorica, 8(3):261277.

Łuczak, T. (1994). On Ramsey minimal graphs. Electron. J. Combin., 1:Research Paper 4, approx. 4 pp. (jornal eletrônico).

Łuczak, T. (2000). On triangle-free random graphs. Random Structures Algorithms, 16(3):260276.

Nešetřil, J. e Rödl, V. (1976). Partitions of vertices. Comment. Math. Univ. Carolinae, $17(1): 85-95$.

Nešetřil, J. e Rödl, V. (1978). The structure of critical Ramsey graphs. Acta Math. Acad. Sci. Hungar., 32(3-4):295-300.

Pak, I. (2001). Mixing time and long paths in graphs. Arquivo eletrônico disponível no endereço http://www-math.mit.edu/ ${ }^{\sim}$ pak/research.html\#r.

Pikhurko, O. (2001). Size Ramsey numbers of stars versus 3-chromatic graphs. Combinatorica, 21(3):403-412.

Pósa, L. (1976). Hamiltonian circuits in random graphs. Discrete Math., 14(4):359-364.

Ramsey, F. (1930). On a problem of formal logic. Proc. London Math. Soc., 48:264-286.

Rödl, V. e Ruciński, A. (1993). Lower bounds on probability thresholds for Ramsey properties.

Em Combinatorics, Paul Erdős is eighty, Vol. 1, páginas 317-346. János Bolyai Math. Soc., Budapest. 
Rödl, V. e Ruciński, A. (1995). Threshold functions for Ramsey properties. J. Amer. Math. Soc., 8(4):917-942.

Rödl, V. e Szemerédi, E. (2000). On size Ramsey numbers of graphs with bounded degree. Combinatorica, 20(2):257-262.

Roth, K. F. (1953). On certain sets of integers. J. London Math. Soc., 28:104-109.

Schrijver, A. (1982). Min-max relations for directed graphs. Em Bonn Workshop on Combinatorial Optimization (Bonn, 1980), páginas 261-280. North-Holland, Amsterdam.

Szemerédi, E. (1975). On sets of integers containing no $k$ elements in arithmetic progression. Acta Arith., 27:199-245.

Szemerédi, E. (1978). Regular partitions of graphs. Em Problèmes combinatoires et théorie des graphes (Colloq. Internat. CNRS, Univ. Orsay, Orsay, 1976), páginas 399-401. CNRS, Paris.

van der Waerden, B. L. (1927). Beweis einer Baudetschen Vermutung. Nieuw Archief voor Wiskunde, 15:212-216.

Woodall, D. R. (1978). Menger and König systems. Em Theory and applications of graphs (Proc. Internat. Conf., Western Mich. Univ., Kalamazoo, Mich., 1976), páginas 620-635. Springer, Berlin.

Younger, D. (1998). Correspondência para Y. Wakabayashi. 


\section{Índice Remissivo}

$(\varepsilon, \vec{G} ; p)$-regular, 62
$(\varepsilon, k, \vec{G} ; p)$-regular, 63
$(\varepsilon ; p)$-regular, 42
$(\eta, D, p)$-esparso, 42
$(\varepsilon, G)$-regular, 35
$(\varepsilon, G ; p)$-regular, 42
$(\varepsilon, k, G)$-eqüipartição, 36
$(\varepsilon, k, G)$-regular, 36
$(\varepsilon, k, G ; p)$-regular, 43
$(b, f)$-expansível, 94
$(p, A)$-uniforme, 93
2 -conexo, 27
$2^{V}, 26$
$C^{r}, 27$
$E(G), 26$
$E_{\bar{G}}(U, W), 62$
$G=G^{n}, 27$
$G[F], 26$
$G_{n, M}, 34$
$G_{n, p}, 33$
$K^{r}, 27$
$K^{r, s}, 27$
$P^{r}, 27$
$V(G), 26$
$\Delta(H), 23$
$\Gamma^{(\ell)}(x), 96$
$\Omega, 30$

$\mathbb{P}, 30$

$\alpha$-magra, 53

$e_{\bar{G}}(U, W), 62$

$\left(\begin{array}{l}V \\ k\end{array}\right), 25$

$\mathcal{G}(n), 33$

$\mathbb{E}, 31$

$\lambda(H), 23$

$\mathcal{C}^{r}, 26$

$\operatorname{Bi}(n, p), 32$

$\operatorname{ex}(G, H), 28$

$\hat{r}\left(H_{1}, \ldots, H_{q}\right), 91$

$\tau(\mathcal{G}), 16$

$d_{G}(v), 26$

$e(G), 26$

$k$-ésimo momento, 31

$k$-PA, 38

$p$-densidade, 42

$p$-densidade orientada, 62

$r$-coloração, 27

$r\left(G_{1}, \ldots, G_{q}\right), 28$

$v(G), 26$

$G[U], 26$

$H \subseteq G, 26$

$[n], 25$

$\chi(G), 27$

$\mathcal{G}(n, M), 34$

$\mathcal{G}(n, p), 33$ 
árvore, 27

arcos, 59

arestas, 26

cópia, 26

caminho, 26

cintura, 27

cintura orientada, 60

circuito, 27

coloração própria, 27

conexo, 27

conjunto excepcional, 36

corda, 27

corte orientado, 59

densidade do par, 35

Desigualdade

de Chebyshev, 32

de Chernoff, 32

de Markov, 31

emparelhamento, 27

espaço amostral, 30

espaço de probabilidade, 30

estrela, 27

evento, 30

eventos elementares, 30

floresta, 27

função de probabilidade, 30

grafo, 26

grafo aleatório, 33

grafo completo, 27

grafo orientado, 59 grafo orientado aleatório, 59

grau de $v, 26$

hipercircuito, 26

hipergrafo, 26

hipergrafo $k$-uniforme, 26

hipergrafo linear, 26

hipergrau, 83

isomorfos, 26

Lema de Regularidade, 36

número cromático, 27

número de ramsey, 28

número de ramsey-aresta, 91

quase certamente, 31

ramsey, 28

ramsey-crítico, 73

ramsey-finito, 73

ramsey-infinito, 73

subdivisão, 93

subgrafo, 26

subgrafo induzido, 26

transversal, 60

transversal de circuitos, 60

transversal de cortes, 60

vértices, 26

valor esperado, 31

variável aleatória, 30

variância, 32

vizinhança, 26 